\title{
On the nature of Hydrogen-rich Superluminous Supernovae
}

\author{
C. Inserra, ${ }^{1,2 \star ~ S . ~ J . ~ S m a r t t ~}{ }^{2}$, E. E. E. Gall ${ }^{2,3}$, G. Leloudas ${ }^{4}$, T.-W. Chen ${ }^{5}$, \\ S. Schulze ${ }^{6,7}$, A. Jerkstrand ${ }^{8}$, M. Nicholl ${ }^{9}$, J. P. Anderson ${ }^{10}$, I. Arcavi ${ }^{11,12,13}$, \\ S. Benetti1 ${ }^{14}$, R. A. Cartier ${ }^{1}$, M. Childress ${ }^{1}$, M. Della Valle ${ }^{15}$, H. Flewelling ${ }^{16}$, \\ M. Fraser ${ }^{17}$, A. Gal-Yam ${ }^{18}$, C. P. Gutiérrez ${ }^{1}$, G. Hosseinzadeh ${ }^{11,12}$, D. A. Howell ${ }^{11,12}$, \\ M. Huber ${ }^{16}$, E. Kankare ${ }^{2}$, T. Krühler ${ }^{5}$, E. A. Magnier ${ }^{16}$, K. Maguire ${ }^{1}$, \\ C. McCully ${ }^{11,12}$, S. Prajs ${ }^{1}$, N. Primak ${ }^{16}$, R. Scalzo ${ }^{19,20}$, B. P. Schmidt ${ }^{21}$, M. Smith ${ }^{1}$, \\ K. W. Smith ${ }^{2}$, B. E. Tucker ${ }^{19,20}$, S. Valenti ${ }^{22}$, M. Wilman ${ }^{16}$, D. R. Young ${ }^{2}$, F. Yuan ${ }^{19,20}$ \\ ${ }^{1}$ Department of Physics and Astronomy, University of Southampton, Southampton, SO17 1BJ, UK \\ 2 Astrophysics Research Centre, School of Mathematics and Physics, Queens University Belfast, Belfast BT7 1NN, UK \\ ${ }^{3}$ Max-Planck-Institut für Astrophysik, Karl-Schwarzschild-Str. 1, DE-85748 Garching-bei-München, Germany \\ ${ }^{4}$ Dark Cosmology Centre, Niels Bohr Institute, University of Copenhagen, Juliane Maries vej 30, 2100 Copenhagen, Denmark \\ ${ }^{5}$ Max-Planck-Institut für Extraterrestrische Physik, Giessenbachstraße 1, 85748, Garching, Germany \\ ${ }^{6}$ Instituto de Astrofísica, Facultad de Física, Pontificia Universidad Católica de Chile, Vicuña Mackenna 4860, 7820436 Macul, Santiago, Chile \\ ${ }^{7}$ Millennium Institute of Astrophysics, Vicuña Mackenna 4860, 7820436 Macul, Santiago, Chile \\ ${ }^{8}$ Max-Planck Institut für Astrophysik, Karl-Schwarzschild-Str. 1, D-85748 Garching, Munich, Germany \\ ${ }^{9}$ Harvard-Smithsonian Center for Astrophysics, 60 Garden Street, Cambridge, Massachusetts 02138, USA \\ ${ }^{10}$ European Southern Observatory, Alonso de CÃşrdova 3107, Casilla 19, Santiago, Chile \\ ${ }^{11}$ Las Cumbres Observatory Global Telescope Network, 6740 Cortona Dr., Suite 102 Goleta, Ca 93117 \\ ${ }^{12}$ Department of Physics, University of California, Santa Barbara, CA 93106-9530, USA \\ ${ }^{13}$ Einstein fellow \\ ${ }^{14}$ INAF, Osservatorio Astronomico di Padova, vicolo dell'Osservatorio 5, 35122, Padova, Italy \\ ${ }^{15}$ INAF Osservatorio Astronomico di Capodimonte, Salita Moiariello 16, I-80131 Napoli, Italy \\ ${ }^{16}$ Institute for Astronomy, University of Hawaii at Manoa, Honolulu, HI 96822, USA \\ ${ }^{17}$ School of Physics, O'Brien Centre for Science North, University College Dublin, Belfield, Dublin 4, Ireland \\ ${ }^{18}$ Department of Particle Physics and Astrophysics, Weizmann Institute of Science, Rehovot 7610001, Israel \\ ${ }^{19}$ Research School of Astronomy and Astrophysics, Australian National University, Canberra, ACT 2611, Australia \\ ${ }^{20}$ ARC Centre of Excellence for All-Sky Astrophysics (CAASTRO), The Australian National University, Canberra, ACT 2611, Australia \\ ${ }^{21}$ Australian National University, Canberra, ACT 2611, Australia \\ ${ }^{22}$ Department of Physics, University of California, Davis, CA 95616, USA
}

Accepted XXX. Received YYY; in original form ZZZ

\begin{abstract}
We present two hydrogen-rich superluminous supernovae (SLSNe), namely SN2013hx and PS15br. These objects, together with SN2008es are the only SLSNe showing a distinct, broad $\mathrm{H} \alpha$ feature during the photospheric phase and also do not show any sign of strong interaction between fast moving ejecta and circumstellar shells in their early spectra. Despite PS15br peak luminosity is fainter than the other two objects, the spectrophotometric evolution is similar to SN2013hx and different than any other supernova in a similar luminosity space. We group all of them as SLSNe II and hence distinct from the known class of SLSN IIn. Both transients show a strong, multicomponent $\mathrm{H} \alpha$ emission after 200 days past maximum which we interpret as an indication of interaction of the ejecta with an asymmetric, clumpy circumstellar material. The spectra and photometric evolution of the two objects are similar to type II supernovae, although they have much higher luminosity and evolve on slower timescales. This is qualitatively similar to how SLSNe I compare with normal type Ic in that the former are brighter and evolve more slowly. We apply a magnetar and an interaction semi-analytical codes to fit the light curves of our two objects and SN2008es. The overall observational dataset would tend to favour the magnetar, or central engine, model as the source of the peak luminosity although the clear signature of late-time interaction indicates that interaction can play a role in the luminosity evolution of SLSNe II at some phases.
\end{abstract}




\section{INTRODUCTION}

Over the past few years, the current generation of wide-field optical surveys have discovered a new class of intrinsically bright transients. They show absolute magnitudes at maximum light of $M_{\mathrm{AB}} \sim-21 \mathrm{mag}$, total radiated energies of order $10^{51}$ erg (e.g. Quimby et al. 2011) and now go by the common name of superluminous supernovae (SLSNe, GalYam 2012). They are factors of 5 to 100 brighter than type Ia or normal core-collapse supernovae and have recently been classified on their spectrophotometric behaviour rather than a simple magnitude threshold (e.g. Papadopoulos et al. 2015; Lunnan et al. 2016; Prajs et al. 2017).

Two distinct groups have emerged so far. The first, and best studied, includes hydrogen-free SLSNe which have spectra at maximum light showing a blue continuum and a distinctive "W"-shaped spectra feature in absorption at $\sim 4200 \AA$. The absorption has been identified as O II and these SNe have been labelled SLSNe I (Quimby et al. 2011; Gal-Yam 2012). Their spectra at about 30 days after peak are very similar to normal or broadlined type Ic SNe at peak luminosity (Pastorello et al. 2010), and hence are also called SLSNe Ic (Inserra et al. 2013b).

The second group is that of hydrogen-rich SLSNe. This includes the very bright, strongly interacting SNe such as SN2006gy (e.g. Smith et al. 2007; Smith \& McCray 2007; Ofek et al. 2007; Agnoletto et al. 2009) - of which the enormous luminosity is mainly powered by the interaction of supernova ejecta with dense circumstellar (CSM) shells and, following the standard taxonomy, should be labeled SLSNe IIn (due to clear hydrogen multicomponent and narrow emission lines exhibited since the early spectra).

However, there is also one known SLSN which in many ways is similar to the type Ic SLSNe, but with a distinct and broad $\mathrm{H} \alpha$ feature. This is SN2008es, which was studied by Miller et al. (2009) and Gezari et al. (2009) and does not obviously show any signs of interaction between fast and slow moving ejecta and CSM shells. The favoured explanation for this object, which somewhat resembled normal luminosity type II SNe (Miller et al. 2009), was a core-collapse explosion of a non-standard progenitor star with a super wind and extended envelope (Gezari et al. 2009). Recent papers have also shown SLSNe I with a weak, but distinct, multi-component $\mathrm{H} \alpha$ emission (Benetti et al. 2014) or with late-time $\mathrm{H} \alpha$ emission (Yan et al. 2015, 2017).

It is plausible that there are multiple powering sources in any of these explosions. For example a central engine may power the bulk of the luminosity, but then interaction between the magnetar powered ejecta and some CSM shells (of varying density) could provide additional energy output and alter the spectra morphology as observed for a nearby sample of slow SLSNe I (Inserra et al. 2017c). Hereafter we will refer to events without obvious spectral signatures of interaction as SLSNe II whereas those with narrow spectral features, multicomponent profiles and obviously powered by interaction will be referred to as SLSNe IIn. In this paper we present two more SLSNe II objects, followed in detail by the Public ESO Spectroscopic Survey for Transient Objects (PESSTO; Smartt et al. 2015) with $\mathrm{H}_{0}=72 \mathrm{~km} \mathrm{~s}^{-1}$, $\Omega_{\mathrm{M}}=0.27, \Omega_{\lambda}=0.73$ adopted as standard cosmology.

\section{SAMPLE}

\section{$2.1 \quad$ SN2013hx}

SN2013hx (SMTJ013533283-5757506) was discovered by the SkyMapper Transient (SMT) and Supernova Survey (Keller et al. 2007; Scalzo et al. 2013) on the 27th December 2013 in $g$ and $r$ bands. Three weeks before the discovery, on MJD 56632.55, a SMT image shows no detection of the transient to $r \simeq 20.28 \mathrm{mag}$. Hence we can determine the epoch of explosion to around twenty days, at least as far as the sensitivity of the images allow. The object coordinates have been measured on our astrometrically calibrated images: $\alpha=01^{\mathrm{h}} 35^{\mathrm{m}} 32^{\mathrm{s}} .83 \pm 0^{\mathrm{s}} .05, \delta=-57^{\mathrm{o}} 57^{\prime} 50.6 " \pm 0$ " .05 (J2000). The object brightened slowly by $\sim 1 \mathrm{mag}$ in the observed $g$-band from discovery to peak and the photometry is given in Table C1. It was classified by the Public ESO Spectroscopic Survey of Transient Objects (PESSTO, Smartt et al. 2015) around maximum as a hydrogen-rich superluminous supernova showing some similarities to SN2010gx (Pastorello et al. 2010) and CSS121015:004244+132827 (Benetti et al. 2014) on February 20 UT (Campbell et al. 2014; Scalzo et al. 2014b). The spectrum showed a blue continuum with broad features in the blue together with a narrow emission feature at $\sim 7500 \AA$ consistent with $\mathrm{H} \alpha$ setting the object at $z=0.125$ (see Table 1 for SN2013hx main properties). It was then immediately selected by PESSTO as followup science targets and a combination of optical, near infrared (NIR) and ultraviolet (UV) photometric monitoring, together with optical spectroscopic monitoring, was carried out (see a summary in Table 2). Observations and data reduction are reported in Appendix A. The Galactic reddening toward the position of the $\mathrm{SN}$ is $\mathrm{E}(\mathrm{B}-\mathrm{V})=0.02 \mathrm{mag}$ (Schlafly \& Finkbeiner 2011). The available spectra do not show Na ID lines from the host galaxy, hence we adopt the Galactic reddening as total reddening.

Deep gri images taken by PESSTO with the New Technology Telescope (NTT)+EFOSC2 on the 10th of December 2015 , after the SN faded, find an extended, faint source at $\alpha=01^{\mathrm{h}} 35^{\mathrm{m}} 32^{\mathrm{s}} .78 \pm 0^{\mathrm{s}} .05, \delta=-57^{\mathrm{o}} 57^{\prime} 52.3 " \pm 0$ " $.05(\mathrm{~J} 2000)$ which is at $2^{\prime \prime}$ from SN location $(4.36 \mathrm{kpc}$ at $z=0.125)$. The magnitudes of this galaxy are $g=24.43 \pm 0.16, r=23.20 \pm 0.16$ and $i=21.82 \pm 0.16 \mathrm{mag}$ (Host A). This source was not detected in the NIR with deep $J, H, K$ images taken by PESSTO with the NTT+SOFI on the 17 December 2015 ( $J>23.1 \mathrm{mag}$, see Table C1 for the limits in each filter). Even deeper images from Magellan+IMACS on the 1 February 2016 showed faint flux closer to the location of SN2013hx (within $0 . \prime 5$ ) at magnitude $g=24.71 \pm 0.38, r=24.55 \pm 0.35$ and $i=23.54 \pm 0.32 \mathrm{mag}$ (Host B). This flux could either be the true host or residual flux from SN2013hx or a combination of both. Given that the Magellan detections are close to $3 \sigma$ significance and that star/galaxy separation is not reliable at these flux limits we avoid any conclusion on the true host (but see Schulze et al. 2016, for an in depth analysis).

\subsection{PS15br}

PS15br was discovered by the Pan-STARRS Survey for Transients (PSST, Huber et al. 2015) ${ }^{1}$ on 16 February

${ }^{1}$ http://star.pst.qub.ac.uk/ps1threepi/psdb/ 
Table 1. Main properties of the SLSNe II here presented.

\begin{tabular}{ccc}
\hline \hline & SN2013hx & PS15br \\
\hline Alternative & SMTJ013533283-5757506 & CSS150226-112519+081418 \\
names & & MLS150612-112519+081418 \\
$\alpha(\mathrm{J} 2000.0)$ & $01^{\mathrm{h}} 35^{\mathrm{m}} 32^{\mathrm{s}} .83$ & $11^{\mathrm{h}} 25^{\mathrm{m}} 19^{\mathrm{s}} .22$ \\
$\delta(\mathrm{J} 2000.0)$ & $-57^{\circ} 57^{\prime} 50.6^{\prime}$ & $8^{\mathrm{o}} 14^{\prime} 18.9^{\prime}$ \\
$z$ & 0.125 & 0.101 \\
Peak $g(\mathrm{mag})$ & -21.70 & -20.22 \\
$\mathrm{E}(\mathrm{B}-\mathrm{V})(\mathrm{mag})$ & 0.02 & 0.06 \\
$\mathrm{~L}_{\text {ugriz }}$ peak (x 10 $0^{43}$ erg s$\left.{ }^{-1}\right)$ & 10.78 & 2.43 \\
Light curve peak (MJD) & $56684.50 \pm 1.00$ & $57089.25 \pm 2.00$ \\
Host $r$ (mag) & $-15.58(\mathrm{~A}),-14.81(\mathrm{~B})$ & -16.25 \\
\hline
\end{tabular}

2015 at $w_{\mathrm{P} 1}=19.10 \pm 0.02 \mathrm{mag}$, confirmed as a transient source the day after and subsequently detected by the Catalina Real-time Transient Survey (CRTS, Drake et al. 2009, with IDs CSS150226-112519+081418 and MLS150612$112519+081418$ ) at $R=18.3 \mathrm{mag}$ on 26 February 2015. The closest pre-detection image is on the 16 January 2015 from CRTS images. We measured the objects coordinates on our astrometrically calibrated images: $\alpha=11^{\mathrm{h}} 25^{\mathrm{m}} 19^{\mathrm{s}} .22 \pm 0^{\mathrm{s}} .05$, $\delta=8^{\circ} 14^{\prime} 18.9^{\prime \prime} \pm 0 " .05(\mathrm{~J} 2000)$. The SN was observed to rise slowly with these multiple detections by PSST and CRTS and was then classified by PESSTO on the 11th of March 2015 (Fraser et al. 2015) as a superluminous supernova around maximum light at $z=0.101$ (see Table 1 for PS15br main properties). As for the previous target, also PS15br was followed-up by the PESSTO consortium and monitored with optical+UV+NIR imaging and optical and NIR (only at late-time) spectroscopy, which revealed the presence of Balmer lines. A summary of PS15br observations can be found in Table 2. Thanks to the additional spectroscopy presented here we were able to secure the classification as SLSN II. The foreground reddening is $\mathrm{E}(\mathrm{B}-\mathrm{V})=0.06 \mathrm{mag}$ from Schlafly \& Finkbeiner (2011). Also in this case the available spectra do not show $\mathrm{Na}$ ID lines from the host galaxy and we only adopt the Galactic reddening.

We retrieved stacks from the Pan-STARRS1 Science Consortium $3 \pi$ survey (Tonry et al. 2012; Schlafly et al. 2012; Magnier et al. 2013) with total exposure times of $g$ (720s), $r$ (590s), $i$ (1800s), $z$ (930s) from before the explosion of PS15br and which are unlikely to contain SN flux (see Appendix C, Table C2). Aperture photometry produced the following $\mathrm{AB}$ magnitudes for the extended host galaxy $g_{\mathrm{P} 1}=22.39 \pm 0.11, r_{\mathrm{P} 1}=22.18 \pm 0.12, i_{\mathrm{P} 1}=21.70 \pm 0.09$, $z_{\mathrm{P} 1}=22.15 \pm 0.11 \mathrm{mag}$. We estimate the centre of this dwarf host galaxy is $0.6^{\prime \prime}$ from SN location (which would be $1 \mathrm{kpc}$ at $z=0.101$ ) and within the uncertainties is effectively coincident. The photometric measurements of the host are in agreement with the Sloan magnitudes of the galaxy SDSS J112519.21+081417.9 listed in the SDSS DR12 (Alam et al. 2015).

\section{PHOTOMETRIC, SPECTROSCOPIC AND POLARIMETRIC EVOLUTION}

\subsection{Light curves}

SN2013hx shows a light curve with a 30 day rise in the observer frame in both $g$ and $r$ bands (see left panel of Fig. 1), reaching an absolute peak magnitude of -21.70 in rest-frame $g$-band. A relatively close non-detections, and a low-order polynomial fit point toward to a rise time of $40 \pm 10$ days ( $\sim 37 \mathrm{~d}$ in rest-frame) from explosion. The rest-frame decline in $g$-band is 2.0 mag $100 \mathrm{~d}^{-1}$ (phase $<10 \mathrm{~d}$ ), while in $V$-band is 4.5 mag $100 \mathrm{~d}^{-1}$ (phase $>20 \mathrm{~d}$ ) suggesting a linear decline

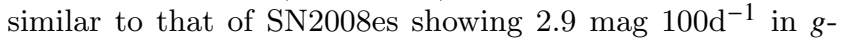
band. This would imply SLSNe II fall on the right/top part of the $s 2 / M_{\max }{ }^{2}$ distribution of type II SNe Anderson et al. $(2014 \mathrm{a})$ and broadly follow the trend $\left(s 2_{\text {SLSNeII }} \approx 2.9 \mathrm{mag}\right.$ and $\left.M_{\max }(S L S N e I I)>-19 \mathrm{mag}\right)$. This would support an association between SLSNe II and normal SNe II. The untimely end of our observational campaign in March 2013 was due to the right ascension of the object ${ }^{3}$. The second season started when it reappeared again in July and PESSTO restarted its annual observing periods in August 2013. Hence the follow-up campaign recovered SN2013hx some five months after maximum light in this second season. From 160d after peak the light curves show a steady decline in all bands.

PS15br was detected during the rise phase, only in the $w$-band of PSST and by the Catalina Sky Survey (CSS). We continued to observe the SN until it disappeared behind the Sun in July 2015 and started again in December 2015. We fit the data with a low-order polynomial and find the peak epoch consistent with MJD 57089.25 \pm 2.00 . Then, the nondetection of the transient 30d before the first detection suggests a rise time of $\sim 35 \mathrm{~d}$ in the rest-frame and hence similar to that of SN2013hx (see right panel of Fig. 1 where $w$-band measurements have been converted in $r$-band through SNAP, see Appendix B). However, the rest-frame peak magnitudes of $-20.22(\mathrm{~g})$ and $<-20.75(U)$ are somewhat fainter than

\footnotetext{
2 We used the s2 parameter since we do not observe a transition from cooling to recombination phase, usually measured with the s1 parameter.

3 Part of these data, mainly an early version of the rest-frame $g$ band and bolometric light curve, were previously given to Nicholl et al. (2015) as a courtesy.
} 
Table 2. Observations log (see Appendix A for further information).

\begin{tabular}{ccc}
\hline \hline Type & phase & SN2013hx \\
\hline \multirow{2}{*}{ Photometry } & early/photospheric $(\leq 110 \mathrm{~d})$ & Skymapper; NTT+EFOSC2; Swift+UVOT; LCO+Sinestro \\
& late/nebular $(>110 \mathrm{~d})$ & NTT+EFOSC2; NTT+SOFI \\
Spectroscopy & early/photospheric $(\leq 110 \mathrm{~d})$ & NTT+EFOSC2; ANU+WiFeS; \\
& late/nebular $(>110 \mathrm{~d})$ & NTT+EFOSC2; VLT+FORS2 \\
\hline \hline \multirow{2}{*}{ Type } & phase & PS15br \\
\hline \multirow{2}{*}{ Photometry } & early/photospheric $(\leq 110 \mathrm{~d})$ & LCO+Sinestro; LT+IO:O; PSST; CSS; Swift+UVOT; NTT+SOFI \\
Polarimetry & late/nebular $(>110 \mathrm{~d})$ & NTT+EFOSC2; LT+IO:O; NTT+SOFI \\
Spectroscopy & early/photospheric $(\leq 110 \mathrm{~d})$ & VLT+FORS2 \\
& late/nebular $(>110 \mathrm{~d})$ & NTT+EFOSC2; ANU+WiFeS; UH+SNIFS \\
\end{tabular}
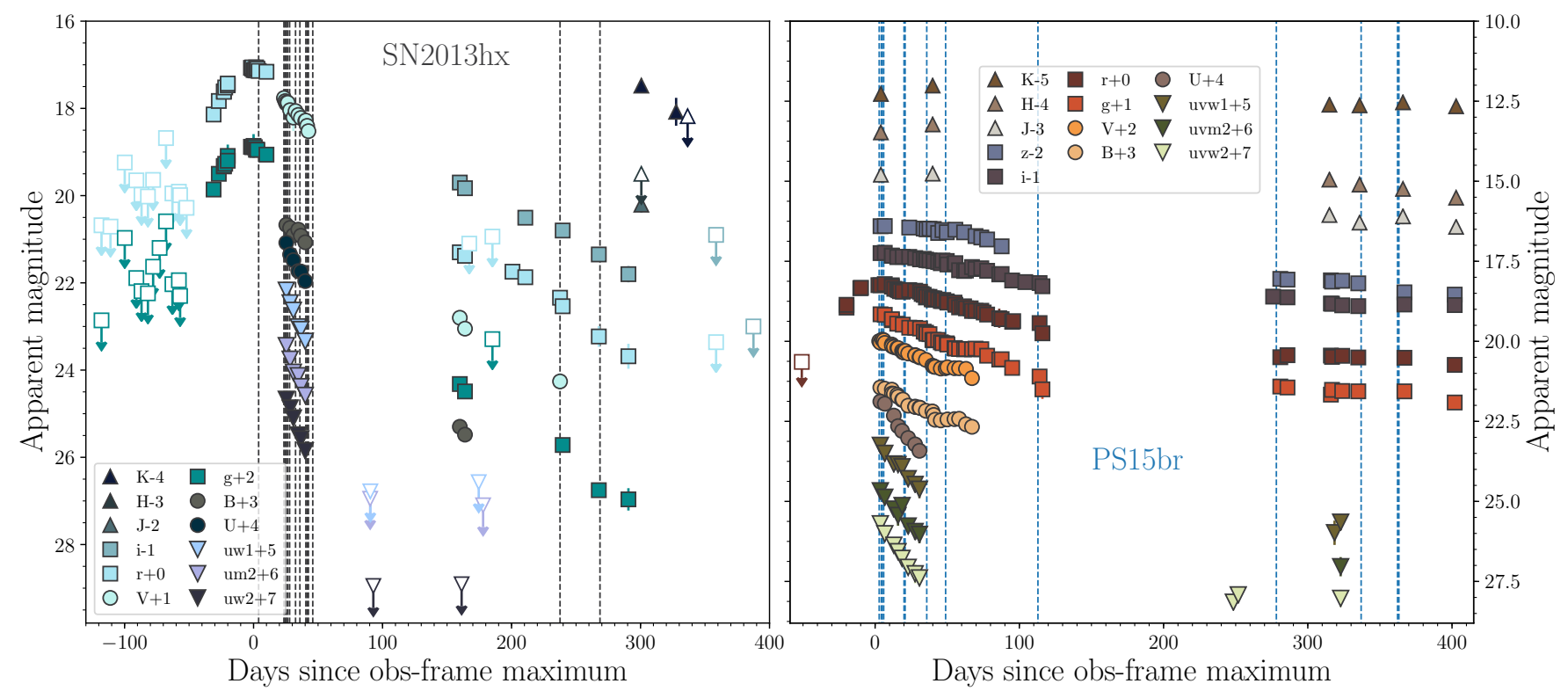

Figure 1. Left: $u v w 2, u v m 2, u v w 1, U, B, V, g, r, i, J, H, K$ light curve evolution of SN2013hx in the observed frame. Right: $u v w 2$, uvm2, uvw $1, U, B, V, g, r, i, z, J, H, K$ light curve evolution of PS15br in the observed frame. Open symbols denote limits. Phase is with respect to maximum light at observed frame in $r$-band. Uncertainties are also reported The epochs of SN2013hx and PS15br spectra are marked with black and blue vertical dashed lines, respectively

those of SN2013hx and SN2008es while its spectroscopic evolution is similar to them (see also Sections $3.2 \& 5$ ). PS15br shows a fairly rapid and linear decline in the UV and $u$ bands after peak, which is gradually less steep in the redder bands. In $B, V, g$ and $r$ bands, the light curves show a slower second decline with $1.2 \mathrm{mag} 100 \mathrm{~d}^{-1}$ in $g$-band between $30-90$ days post peak in rest-frame. Last two photospheric epochs of PS15br could suggest a faster decrease similar to what experienced by some type II at similar phase (see Valenti et al. 2015), which could correspond to the end of hydrogen recombination. In the second season (phase $>250 \mathrm{~d}$ ) the light curves show a very slow, almost flat, behaviour in all bands. This is the consequence of interaction between the SN ejecta and CSM material similar to that experienced by SN2013hx (see Section 5).

Due to PS15br fainter peak luminosity with respect to the other two objects, it is important to understand how it compares with the few hydrogen-rich SNe populating a similar absolute luminosity space. PS15br shows an overall slower evolution than other objects at similar absolute magnitude ( $\gtrsim-19.5$ in $g$-band, Arcavi et al. 2016), suggesting as PS15br is different from the transients showing peak magnitude between those of normal type II and SLSNe. The only other non-SLSN object displaying a somewhat similar brightness is the type IIn/IIL SN2013fc $\left(\mathrm{M}_{g} \sim-20.2\right.$, Kangas et al. 2016), which however decreases of $>3.1 \mathrm{mag}$ $100 \mathrm{~d}^{-1}$ in $g$-band in the first 90 days. Moreover, SN2013fc displays a similar spectroscopic behaviour to type II suggesting a CSM interaction as source for its bright peak luminosity. Although PS15br peak luminosity is closer to the range between SLSN and normal type II SNe, it shows a photometric behaviour and spectroscopic evolution different than the objects presented in Arcavi et al. (2016) and (Kangas et al. 2016). 


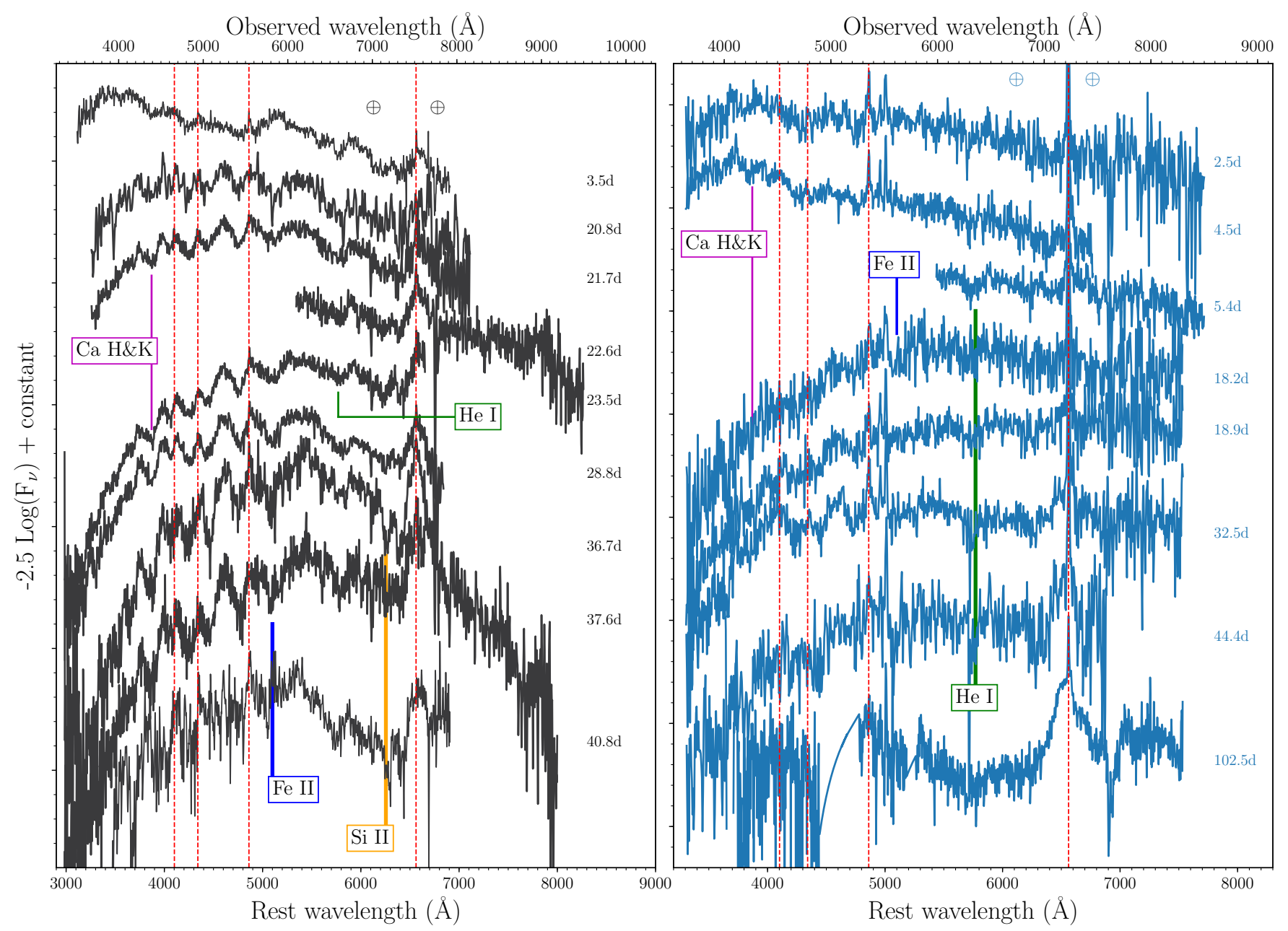

Figure 2. Left: selection of photospheric spectra evolution of SN2013hx. Right: selection of spectra evolution of PS15br. The phase of each spectrum relative to light curve peak in the rest frame is shown on the right. The spectra are corrected for Galactic extinction and reported in the rest frames. The most prominent features are labeled. Balmer lines $\mathrm{H} \alpha$ to $\mathrm{H} \delta$ are marked with red vertical dashed lines. The $\oplus$ symbols mark the positions of the strongest telluric absorptions. WiFeS spectra were convolved with a factor of five and subsequently binned to a $5 \AA$ scale.

\subsection{Spectroscopy}

The spectra evolution of the two SNe is shown in Fig. 2 (cfr. Table C7). Both SN2013hx (left panel of Fig. 2) and PS15br (right panel of Fig. 2), like SN2008es (Gezari et al. 2009; Miller et al. 2009), do not show the typical broad absorption features of O II that we see in SLSNe I. We note that the spectra have temperatures consistent with the photospheric temperatures at which these ionic transitions are prominent (12000 - $16000 \mathrm{~K}$, Quimby et al. 2013; Inserra et al. 2013b), even though such ions could be sensitive to non-thermal radiation in a way similar to He I (Mazzali et al. 2016). In general the post peak spectra of both objects show Balmer lines, $\mathrm{He}, \mathrm{Ca}, \mathrm{Fe}$ and other metal lines.

The first spectrum of SN2013hx, 3 days after peak, shows a broad $\mathrm{H} \alpha$ and $\mathrm{He}$ I $\lambda$ 15876, while that of 21 days past peak also shows conspicuous Balmer lines and an emission feature at $\sim 4600 \AA$ similar to those observed in PS15br and SN2008es (see Fig. 3). This feature was also present in the first spectrum but weaker. The emission peak of this fea- ture could be again associated with C III/N III, possibly even contaminated by Fe III forest lines rather than He II $\lambda 4686$ (see Fig. 3). All the Balmer lines have a P-Cygni profile with the exception of $\mathrm{H} \alpha$ showing only the emission component up to $\sim 30$ days after peak. However, the absorption component exhibited by $\mathrm{H} \alpha$ in the +36 to +38 day spectra is weak. Such behaviour resembles that of fast-declining type II SNe (or type IIL). A transient unresolved narrow $\mathrm{H} \alpha$ line tentatively detected in our first spectrum may point towards some weak interaction at early times, however such behaviour is seen in many type II SNe at early times (e.g. Fassia et al. 2001; Gal-Yam et al. 2007; Inserra et al. 2013a; Gutiérrez et al. 2017a). A shallow He I $\lambda 5876$ line, possibly blended with $\mathrm{Na}$ ID, is visible until 40 days after maximum. The Ca $\mathrm{H} \& \mathrm{~K}$ lines are visible from +20.8 days, as well as a feature in absorption at $\sim 5000 \AA$ likely related to the Fe II multiplet $\lambda \lambda 4924,5018,5169$. Two other P-Cygni profiles are visible from 20 to 40 days after peak at $3745 \AA$ and $4575 \AA$ which, based on the similarities with type II SNe, we identify as Fe I. They are possibly blended with Ti II, and Fe II, respectively. 


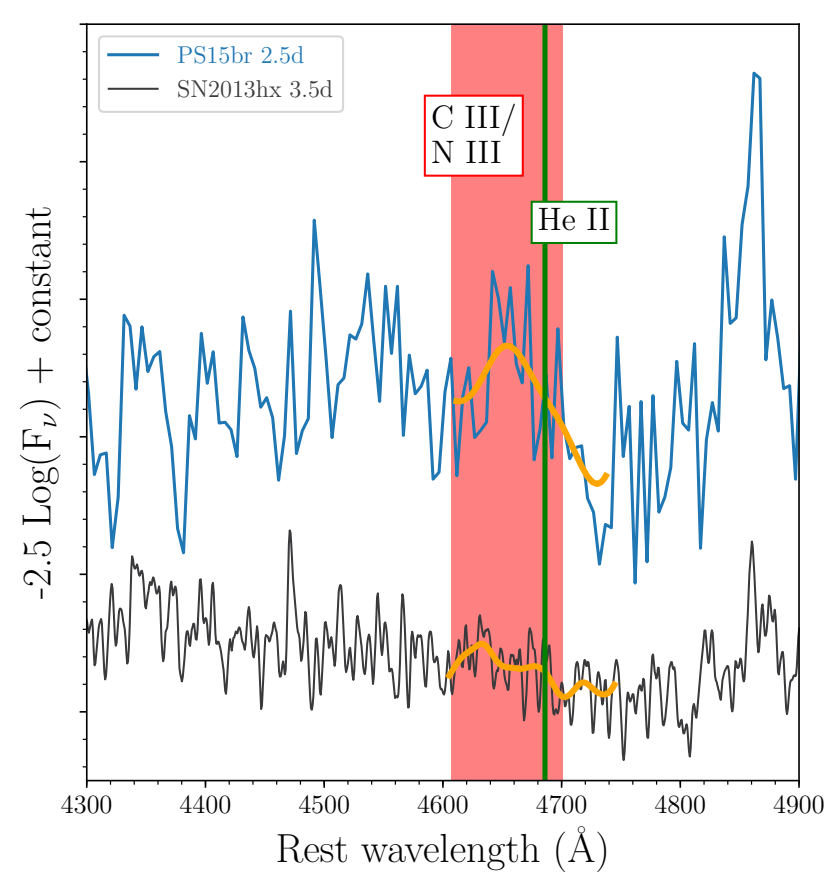

Figure 3. First spectrum of each SN in the region around $4600 \AA$ with the rest-frame He II $\lambda 4686$ marked by a green vertical line and the region of $\mathrm{C}$ III/N III lines by a red area. A gaussian filter (in orange) has been applied to highlight the wavelength region of interest.

An absorption feature is visible from $+23 \mathrm{~d}$ to $+38 \mathrm{~d}$ in SN2013hx (and less clearly from $+5 \mathrm{~d}$ to $+19 \mathrm{~d}$ in PS15br) on the blue side of $\mathrm{H} \alpha$ with an absorption minimum at $6250 \AA$. This absorption feature has been observed in several type II (Chugai et al. 2007; Inserra et al. 2011, 2012a,b, 2013a; Valenti et al. 2014, 2015; Bose et al. 2015) at both early or late photospheric epoch and goes by the name of 'cachito' (Gutiérrez et al. 2017a). The two interpretations are high velocity hydrogen originating from interaction between rapidly expanding SN ejecta and a CSM - or Si II $\lambda 6355$. The latter seems the most plausible identification in our objects since it shows a velocity consistent with, although slightly lower than, other metal lines such as Fe II (see Section 4.4).

In the earliest spectrum of PS15br there is a feature at $4600 \AA$ that could be associated with $\mathrm{C}$ III/N III rather than He II $\lambda 4686$ (see Fig. 3). This feature already weakens in the spectrum at 5 days after maximum and has been observed in several non-superluminous type II (e.g. SNe 1998S, 2007pk, 2009bw, 2013cu; Fassia et al. 2001; Inserra et al. 2012a, 2013a; Gal-Yam et al. 2014). PS15br never shows a broad $\mathrm{H} \alpha$ absorption component but displays a multicomponent emission profile due to the combination of a relatively broad feature $\left(\mathrm{v} \sim 1500 \mathrm{~km} \mathrm{~s}^{-1}\right)$ and a narrow component ( $\sim 110$ $\mathrm{km} \mathrm{s}^{-1}$ ). This is likely due to the host galaxy since it has a similar width to those of the [O III] emission lines of the host galaxy at $\lambda 4959\left(\mathrm{v} \sim 110 \mathrm{~km} \mathrm{~s}^{-1}\right)$ and $\lambda 5007\left(\mathrm{v} \sim 100 \mathrm{~km} \mathrm{~s}^{-1}\right)$ as measured from our WiFeS spectrum at early time $(+4 \mathrm{~d})$ and Xshooter spectra at late time (337 and 362 days after maximum). This highlights that there is no evolution in the strength of the line and it supports their identification as galaxy line since are comparable to those of the host galax- ies of SLSNe in previous studies (e.g. Leloudas et al. 2015a). $\mathrm{H} \beta$ has a similar behaviour although the absorption component is visible from 20 days after maximum. Ca II $\mathrm{H} \& \mathrm{~K}$ is visible in the first spectrum and becomes more prominent from day 18 when the metal lines appear as shown by the presence of the Fe II multiplet $\lambda \lambda 4924,5018,5169$ and possibly Fe I that replaces the $4600 \AA$ feature. He I $\lambda 5876$, or a combination of this element with $\mathrm{Na}$ ID $\lambda \lambda 5890,5896$, is detectable in the first spectrum and clearly from the third, showing a P-Cygni profile.

The last three PS15br spectra of the photospheric phase (32 to 102 days) show the appearance of a broad component (FWHM $\sim 8000 \mathrm{~km} \mathrm{~s}^{-1}$ ) at $\mathrm{H} \alpha$ which is strongly asymmetric in the final photospheric spectrum at 102 days (see Fig. 2). The centroid of the broad $\mathrm{H} \alpha$ feature is somewhat obscured by the narrow galactic emission line, but is certainly blue shifted and we estimate its peak is at $-750 \mathrm{~km} \mathrm{~s}^{-1}$. The asymmetric blue emission is common for $\mathrm{H} \alpha$ of type II SNe and could be due to an opaque core concealing the outermost layers of the receding part of the envelope where the $\mathrm{H} \alpha$ is formed (Chugai 1985). Alternatively it may be due to a steep density profile of the hydrogen layer, which increases the probability to observe blue-shifted photons since the opacity is mainly due to electron scattering and the line emission mostly comes from the region below the continuum photosphere (Dessart \& Hillier 2005; Anderson et al. 2014b).

Considering the slower light curve decline of PS15br compared to SN2013hx and SN2008es, the formation of the early PS15br $\mathrm{H} \alpha$ profile is likely to be related to a weak interaction occurring at early epochs, whereas the SN ejecta would be responsible for the profile of late epochs. PS15br is not a textbook interacting supernova since other ions, such as $\mathrm{Fe}$ II and $\mathrm{Ca} \mathrm{H} \& \mathrm{~K}$, are observed with a P-Cygni profile during its photospheric evolution. The spectrophotometric early behaviour suggests an interaction with a nearby dense circumstellar medium that is swept up in the first week after maximum light. This behaviour is observed in many $\mathrm{SNe}$ II (Yaron et al. 2017; Morozova et al. 2017; Moriya et al. 2017), which show a transition from spectra with signs of weak interactions at early time to typical type II later on (14-30 days after explosion). Combining such spectroscopic evidences with the slow light curve decline, we suggest that PS15br is a more luminous version of a transitional type II, but still different with respect to luminous IIn (e.g. Smith et al. 2007) showing a distinct spectrphotometric evolution.

The spectral evolution of these objects is similar (in the observed ions and their strength) and provides additional information about hydrogen-rich SLSNe.

\subsection{Imaging polarimetry}

On 2015 March 14 UT 05.2, corresponding to a rest-frame phase of 6 days after maximum, we obtained broadband polarimetry of PS15br with VLT/FORS2 using the $V$ filter ${ }^{4}$. We observed it in four different half-wave retarder plate angles: $0,22.5,45$ and $67.5 \mathrm{deg}$. The exposure time was $150 \mathrm{~s}$

4 Unfortunately only one epoch was obtained due to a combination of decreasing apparent magnitude and ending of the ESO semester. 


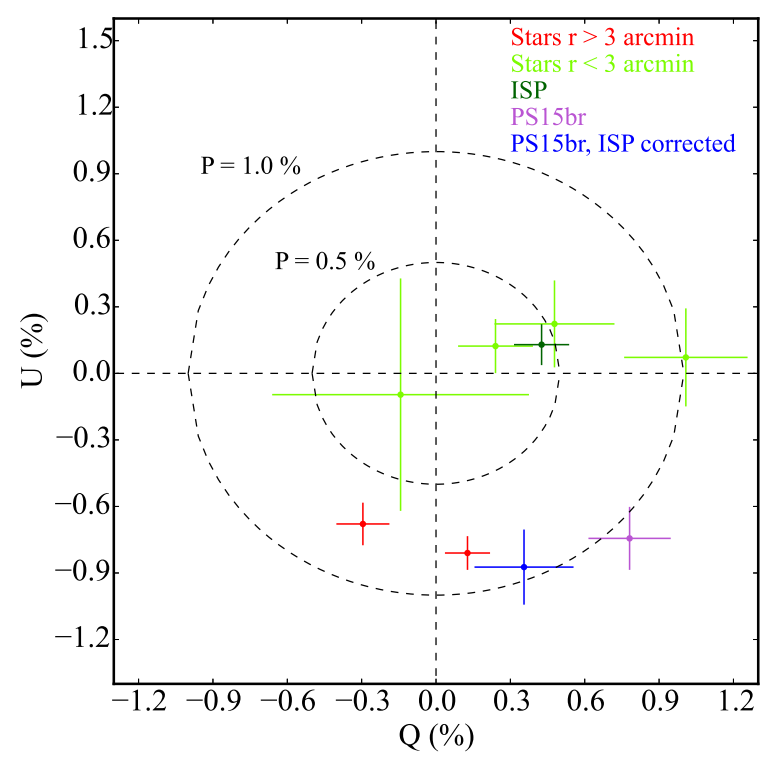

Figure 4. Q-U plane of PS15br at $+6 \mathrm{~d}$ from maximum light. Concentric circles show polarization degrees of $0.5 \%$ and $1.0 \%$.

for each angle. The data were reduced in a standard manner by using bias and flat frames, without polarisation units in the light path. We obtained PSF photometry of PS15br and six field stars in the field of view (FOV) and determined their Stokes parameters. The measurements were corrected for instrumental polarisation (Patat \& Romaniello 2006) and interstellar polarisation (ISP). For more details on the reductions and analysis, see Leloudas et al. (2015b).

Fig. 4, shows the location of PS15br and the field stars on the $Q-U$ plane, after correcting for instrumental polarisation. The error bars that are shown are the measurement errors resulting from the PSF fitting and essentially depend on the $\mathrm{S} / \mathrm{N}$ ratio ( $\sim 500$ for PS15br). The field stars (light green and red points) present a significant scatter in this plane and the SN signal (purple) is comparable to the one of the field stars. It is therefore likely that the signal of the field stars and the SN is dominated by ISP. Patat \& Romaniello (2006) showed that the instrumental-induced polarisation in FORS1 - an instrument with identical design to that of FORS2 - has a radial pattern with polarisation increasing as we move away from the optical axis. The two field stars that are the main cause of the scatter (red) are at the outskirts of the FOV, more than $3.6^{\prime}$ from PS15br, which is in the centre of the FOV. On the other hand, the measurement of the stars closer to the object (light-green) are self-consistent with each other and can be used for a more accurate determination of the ISP.

The ISP is shown in Fig 4 as the weighted average of the light-green stars. Therefore, despite the fact that all points have been corrected for instrumental polarisation - using the FORS1 relation in Patat \& Romaniello (2006) - it is possible to obtain a self-consistent picture by only using field stars closer to the optical axis and the $\mathrm{SN}$. These result in a consistent determination of the ISP $\left(Q_{\text {ISP }}=0.42 \pm 0.11 \%, U_{\text {ISP }}=0.13 \pm 0.09 \%\right)$, and are clearly

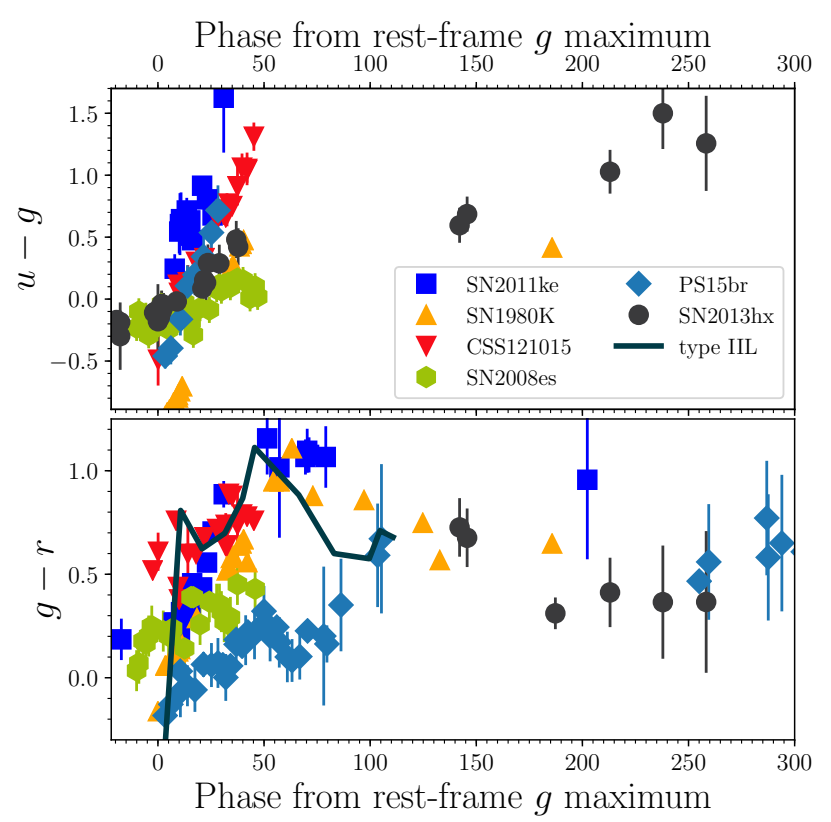

Figure 5. Comparison of the dereddened and $K$-corrected colour evolutions. SN2013hx and PS15br are shown together with the other SLSN II SN2008es (Gezari et al. 2009; Miller et al. 2009), SLSNe I SN20011ke (Inserra et al. 2013b) and CSS121015 (Benetti et al. 2014) together with type II SN1980K, for which the colour curve is actually $U-B$ (top panel) and $B-V$ (bottom panel), and that of a type IIL template from Sanders et al. (2015).

different from the properties of PS15br $\left(Q_{\mathrm{SN}}=0.78 \pm 0.17 \%\right.$ and $U_{\mathrm{SN}}=-0.74 \pm 0.14 \%$.

By correcting for the ISP so evaluated, we obtain a significant polarisation signal $Q=0.36 \pm 0.20 \%$ and $U=$ $-0.87 \pm 0.17 \%$, resulting in $P=0.94 \pm 0.17 \%$. After correcting for polarisation bias, according to Patat \& Romaniello (2006), this value reduces to $P_{0}=0.93 \pm 0.17 \%$. Indicatively, this level of polarisation would correspond to an asymmetry of $\mathrm{E} \sim 10-15 \%$, assuming an ellipsoidal photosphere (Hoflich 1991). Such level of polarisation is consistent with that reported for SLSN I SN2015bn at similar epochs (Inserra et al. 2016b; Leloudas et al. 2017) but higher than that of SLSN I LSQ14mo (Leloudas et al. 2015b), for which no evidence for significant deviation from spherical symmetry was found. On the other hand, such measurement is higher than those reported for type II SNe soon after peak (see Wang \& Wheeler 2008 , for a review) and a factor of two less than that of type IIn (e.g. SN1998S, Wang et al. 2001), which are however dominated by strong $\mathrm{H}$ emission lines not visible in PS15br.

However, of we assume that all field stars are equally good for the determination of the ISP, we conclude that there is a significant scatter - probably pointing to an unaccounted source of systematic error - and that no conclusive evidence on the source of the polarisation associated with PS15br can be derived. Unfortunately, there are no other suitable stars (bright and not saturated) in the FOV of PS15br to resolve this uncertainty. 


\section{SLSNE II INTO CONTEXT}

\subsection{Colour curves}

Since colour curves are useful probes of the temperature evolution of supernovae, we computed the rest-frame curves, after accounting for the reddening and redshift effects of timedilation and $K$-correction. In Fig. 5, SN2013hx and SN2008es show a constant $u-g$ increase toward redder values. However, PS15br is $\sim 0.25$ mag bluer than other SLSNe II at peak. Its colour evolution is also steep, but similar to the prototypical SLSN I SN2011ke and CSS121015, a SLSN I showing early interaction with a H-shell. This could support the suggestion of an early, weak interaction in PS15br as observed in several transitional type II. We also note that after 20 days, the $u-g$ colour evolution gradient of SN2013hx is similar to that of fast-declining type II (or type IIL, $u-g \sim 0.4 \mathrm{mag}$ ), whereas PS15br is slightly steeper and SN2008es slightly shallower. Although the former is, overall, shifted redwards.

SLSNe II (PS15br and SN2008es) $g-r$ colours show a similar behavior with a slow, monotonic increase toward redder colours until 50 days. SLSNe II do not show the constant colour exhibited by SLSNe I from the pre-peak phase to $\sim 15 \mathrm{~d}$ (Inserra et al. 2013b). In general the colours evolve more slowly to the red than type IIL SNe, which cool down to $g-r \sim 0.6$ in the first 20 days as shown by a template colour evolution (Sanders et al. 2015). We note that although such template is derived from type II, peak luminosity and light curve evolution of the objects are similar to type IIL similarly to other sample papers using Bessell filters (e.g. Li et al. 2011; Faran et al. 2014; Galbany et al. 2016; Valenti et al. 2016). In addition, it appears that PS15br $g-r$ shows a turnover at 50 days and evolves bluewards before turning to the red again. However, considering the uncertainties and compare the colour curve with the temperature behavior (see Section 4.4) it may be a flattening in the colour evolution (at $g-r \sim 0.24 \mathrm{mag}$ ) rather than a blueward evolution. The object then clearly evolves to the red afterwards and with $g-r=0.6 \mathrm{mag}$ after $100 \mathrm{~d}$ it appears similar to SN2013hx and type II SNe. After 200 days SN2013hx and PS15br colour curves remain steady at $g-r=0.4 \mathrm{mag}$ and $g-r=0.6 \mathrm{mag}$ in a similar fashion to that experienced by normal type II/IIL such as SN1980K.

\subsection{Bolometric luminosity}

UV to NIR photometry is required to obtain a direct measurement of the full bolometric luminosity. This is typically difficult to achieve at all epochs during a SN evolution. Despite the lack of data, valid corrections can be applied to the observed photometric bands to compute the total bolometric flux (e.g. Pastorello et al. 2015; Chen et al. 2015; Kangas et al. 2016; Inserra et al. 2017c, for a further insight).

The effective temperatures of the photosphere of SLSNe II during their first 30-50 days after explosion are between $T_{\mathrm{bb}} \sim 10000-16000 \mathrm{~K}$ (see Section 4.4). This means that their fluxes peak in the UV $(\lambda<3000 \AA)$ during this period while our rest-frame ugri bands typically cover from $3500 \AA$ redwards. Thus a significant fraction of the flux is not covered by the optical ugri imaging, as testified by the UV to total luminosity ratio for SN2008es and PS15br (see middle panel of Fig. 6). At around 20d after peak, the effective temperatures tend to drop below $10000 \mathrm{~K}$, hence the SEDs peak between $3000 \AA$ and $4000 \AA$. Although the peak of the SED moves redward, a significant amount of the bolometric flux is radiated in the UV even during these late stages. In the following, we will use the term 'pseudo-bolometric light curve' to refer to a bolometric light curve determined using only the optical filters with the flux set to zero outside the observed bands.

To calculate bolometric luminosity, the broad band magnitudes in the available optical bands were converted into fluxes at the effective filter wavelengths, then were corrected for the adopted extinctions (cfr. Section 2). A SED was then computed over the wavelengths covered and the flux under the SED was integrated assuming there was zero flux beyond the integration limits. Fluxes were then converted to luminosities using the distances previously adopted. We initially determined the points on the pseudobolometric light curves at epochs when ugri were available simultaneously (or very close in time) and later for epochs with coverage in less than the four filters. Magnitudes from the missing bands were generally estimated by interpolating the light curves using low-order polynomials $(n \leq 3)$ between the nearest points in time. For some points this interpolation was not possible and hence we extrapolated the magnitudes assuming constant colours from neighbouring epochs.

Since SN2013hx and SN2008es have similar optical light curves we built the SN2013hx bolometric light curve considering the same NIR flux contribution of SN2008es at early epochs (SN2008es broad band data were retrieved by Miller et al. (2009); Gezari et al. (2009)). To check the validity of this assumption we also estimated the bolometric light curve integrating, from UV to NIR wavelengths (1000 $\AA$ $25000 \AA$ ), our best blackbody fit to the available SN2013hx SED and found similar NIR flux contribution to the bolometric light curve to that of the previous method. The difference between these two methods is included as uncertainty in the evaluation of the SN2013hx bolometric light curve. In Fig. 6 (middle panel) we compared UV and NIR contributions to the total bolometric luminosity of SN2013hx and PS15br with that of SN2008es. The UV contributions are similar and drop almost to zero after 60 days from maximum, while the NIR contribution increases with time. We have late NIR data in $J H K$ information for SN2013hx and PS15br, whereas SN2008es only has data in the $J$ and $H$ bands at these epochs where the NIR contribution becomes significant. If we were to ignore the PS15br $K$-band contribution, the two percentages of NIR contribution ( $J H$ of PS15br and SN2008es) are similar. Therefore when we evaluated the full bolometric light curves of SN2013hx and SN2008es we added the $K$-band contribution estimated specifically by PS15br NIR data.

The peak luminosities of our bolometric light curves are $\mathrm{L}_{\mathrm{SN} 2013 \mathrm{hx}} \approx 2.75 \times 10^{44} \mathrm{erg} \mathrm{s}^{-1}, \mathrm{~L}_{\mathrm{PS} 15 \mathrm{br}} \approx 4.15 \times 10^{43} \mathrm{erg} \mathrm{s}^{-1}$ and $\mathrm{L}_{\mathrm{SN} 2008 \mathrm{es}} \approx 3.00 \times 10^{44} \mathrm{erg} \mathrm{s}^{-1}$. While the maximum luminosities reached by the pseudo-bolometric light curves are almost factor two lower. We note that SN2008es values are in agreement with those previously reported by Gezari et al. (2009); Miller et al. (2009).

The bottom panel of Fig. 6 compares the pseudobolometric light curves ugriz of our sample of SLSNe II namely SN2013hx, SN2008es, PS15br - with that of the peculiar SLSN I CSS121015, a prototypical SLSN I (SN2011ke) 


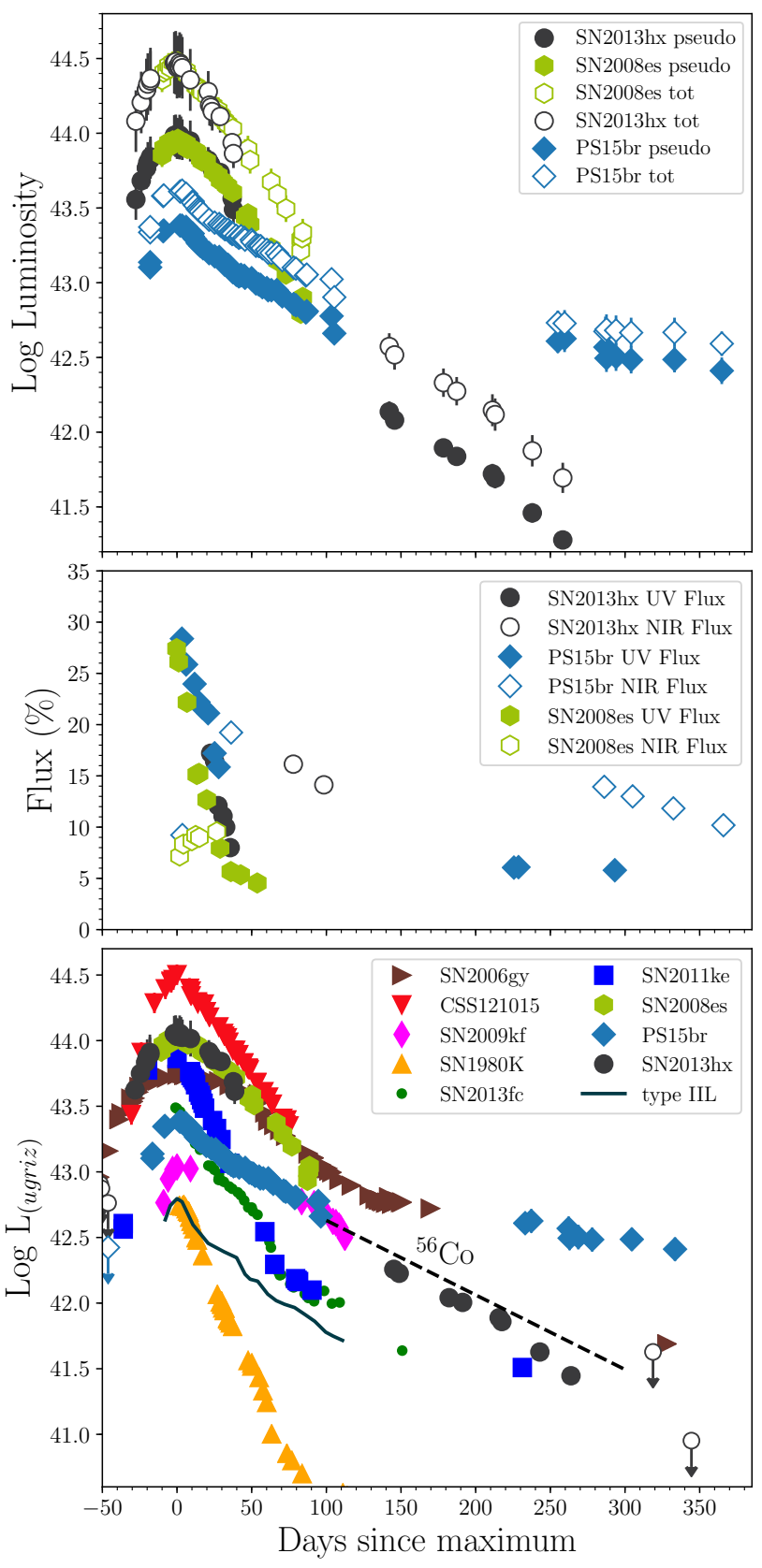

Figure 6. Top: pseudo (filled symbols) and proper bolometric (open symbols) of three SLSNe II. Middle: percentage of the bolometric flux in UV (filled symbols) and NIR (open symbols) with respect to the total bolometric flux derived from data of SLSNe II. Bottom: comparison of pseudo bolometric light curves (ugriz) of SN2013hx, PS15br and SLSN II 2008es (Gezari et al. 2009; Miller et al. 2009), the interacting SLSN IIn 2006gy (Smith et al. 2007), the prototypical SLSN I SN2011ke (Inserra et al. 2013b) and the early interacting SLSN I CSS121015 (Benetti et al. 2014), the type II SNe 1980K (Barbon et al. 1982) and 2009kf (Botticella et al. 2010), the bright and mild interacting type II $2013 \mathrm{fc}$ (Kangas et al. 2016), as well as a type IIL template (Sanders et al. 2015). These bolometric light curves are computed after correcting the observed broadband photometry for time dilation and applying $K$-corrections, through SNAKE (see Appendix B), and extrapolating up to $z$ band when this was unavailable. The black dashed line is the slope of ${ }^{56} \mathrm{Co}$ to ${ }^{56} \mathrm{Fe}$ decay. and type II SNe. We note that the extrapolation up to $z$ band has been done assuming constant colour from neighbouring epochs or integrating the best fit blackbody to the available SED up to the $z$ band coverage. The comparison remarks that light curves of SN2013hx and SN2008es are notably similar. CSS121015 also exhibits a fairly similar decline over the first 40 days, but is 0.5 dex brighter than any other SN. SN2013hx rise appears slower than typical SLSN I events like SN2011ke. PS15br is $\sim 0.5$ dex fainter than the other two SLSNe II and sits in the gap between normal luminosity type II SNe and SLSNe II. Although PS15br exhibits a similar decline to SN2008es and SN2013hx over the first 30 days, it clearly does not show the faster decline observed after this epoch - it remains on quite a linear decline for nearly 100 days. During this time baseline it also shows a similar overall decline rate similar to that of one of the brightest type II, SN2009kf (Botticella et al. 2010). PS15br is slower than the interacting SN2013fc (Kangas et al. 2016), which is the only type II reaching a luminosity similar to those of PS15br due to its early interaction, PS15br decline of $\sim 0.4$ dex over the first 40 days after peak is similar to that shown by the type IIL template on a similar timescale, but slower afterwards.

The comparison shows that both SN2013hx and SN2008es have fairly similar shaped profiles within 10-20 days of peak. To make quantitative comparisons of the light curve widths, we measured a width when the pseudobolometric flux is $1 / e$ less than that at maximum light in order to match the estimates of the diffusion timescale parameter $\tau_{m}$ in Nicholl et al. (2015). When these epochs were not specifically covered by a data point, we initially used low order polynomial fits $(n \leq 4)$ to interpolate and find the width (see Fig. 7). The values are reported in Table 3. We note that the values are slightly different than those reported in Nicholl et al. (2015), that could be due to small difference in the evaluation of the bolometric light curves. The uncertainties reported are estimated from the differences between the values achieved with different polynomials orders and time baselines. We also employed Gaussian processes (GP), which are generic supervised learning methods widely used in the machine learning community (Bishop 2006; Rasmussen \& Williams 2006) for Bayesian regression and classification problems and successfully used in the context of SN (e.g. Kim et al. 2013; Scalzo et al. 2014a; de Jaeger et al. 2017) and SLSN light curve fitting (Inserra et al. 2017a). An important advantage of GP regression compared to other regression techniques is that it produces a best-fit model together with uncertainty at each point and a full covariance estimate of the result at unknown points (Ivezic et al. 2014). We used the machine learning packages GEORGE (Ambikasaran et al. 2014) written in PYTHON and a Matern $3 / 2$ kernel to fit our light curve width (see Inserra et al. 2017a, for further details about the methodology, the kernel and package choice). The results are reported in Table 3, while a comparison with the polynomial fitting can be seen in Fig. 7. Since the two methods seems overall comparable, hereafter we will refer to the GP results and not those of the polynomial fitting.

As highlighted before, the decline of SLSNe II tend to be slower than that of SLSNe I such as SN2011ke (a prototypical SLSN I), while the rise time is comparable for all objects. The $1 / e$ width is $\gtrsim 70$ days for SLSNe II and around 50 days 
Table 3. Width of SLSNe pseudo-bolomotric ugriz light curves. P refers to the polynomial measurements, while GP to those performed with Gaussian processes.

\begin{tabular}{lcccccc}
\hline \hline SN & $\begin{array}{c}\text { width }(\mathrm{P}) \\
\text { (day) }\end{array}$ & $\begin{array}{c}\text { rise }(\mathrm{P}) \\
\text { (day) }\end{array}$ & $\begin{array}{c}\text { decline }(\mathrm{P}) \\
\text { (day) }\end{array}$ & $\begin{array}{c}\text { width (GP) } \\
\text { (day) }\end{array}$ & $\begin{array}{c}\text { rise (GP) } \\
\text { (day) }\end{array}$ & $\begin{array}{c}\text { decline (GP) } \\
\text { (day) }\end{array}$ \\
\hline SN2013hx & $64 \pm 3$ & 27 & 37 & $69 \pm 8$ & $31 \pm 7$ & $38 \pm 4$ \\
PS15br & $80 \pm 5$ & 20 & 60 & $83 \pm 4$ & $23 \pm 4$ & $60 \pm 4$ \\
SN2008es & $67 \pm 5$ & 24 & 43 & $70 \pm 16$ & $30 \pm 16$ & $40 \pm 4$ \\
SN2011ke & $48 \pm 3$ & 24 & 24 & $47 \pm 4$ & $18 \pm 3$ & $29 \pm 2$ \\
\hline
\end{tabular}
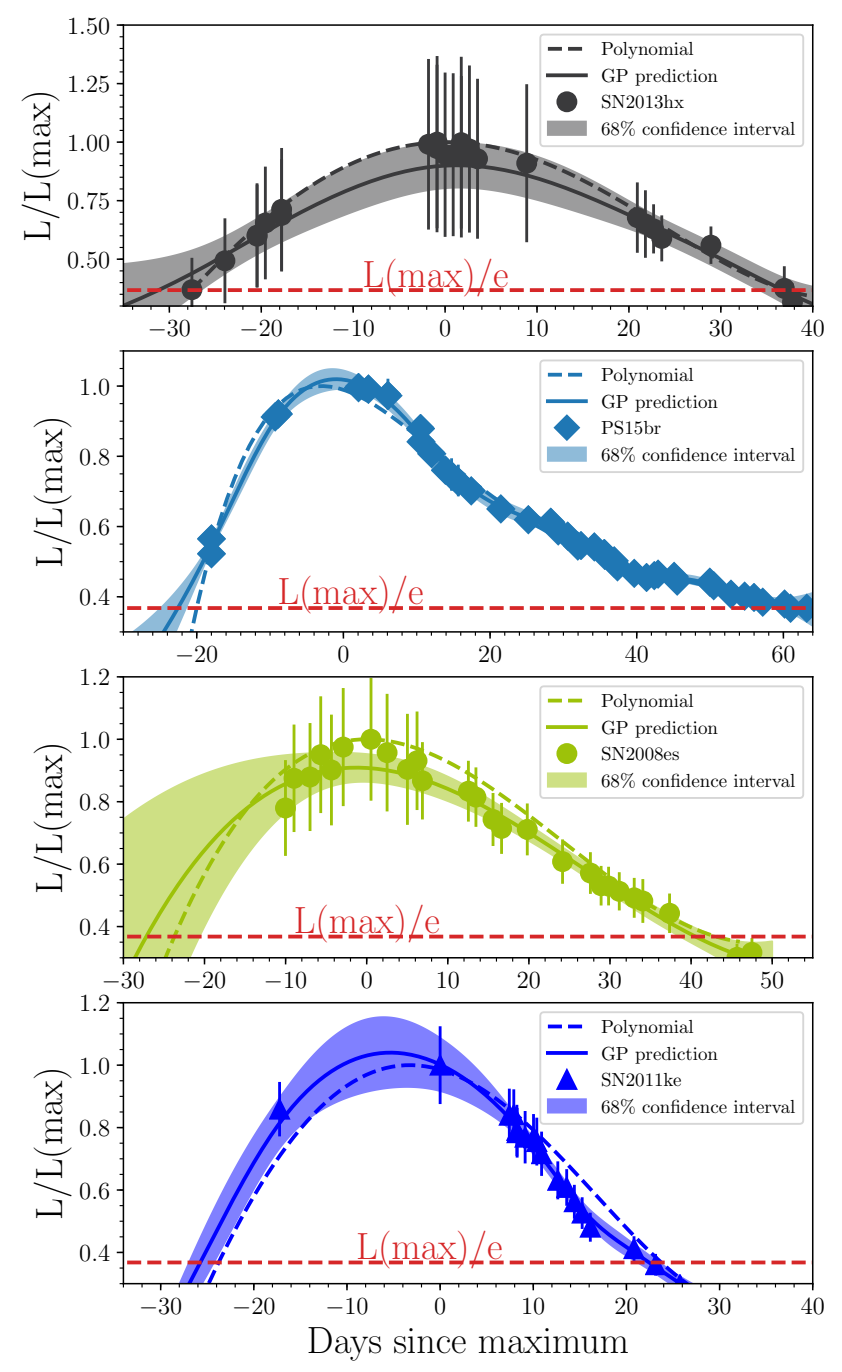

Figure 7. Pseudo bolometric light curves (ugriz) normalized to the maximum of the data, which is at zero days. The luminosity equivalent to $\mathrm{L}(\max ) / \mathrm{e}$ is highlighted by a red dashed line. We note that the GP peak for SN2008es and SN2011ke occurs earlier in time than that of the polynomial and data

for SN2011ke. We used the same techniques to measure the width of CSS121015 (SLSN I interacting with a hydrogen shell) and found it to be similar to that of SN2011ke. That is a consequence of the sharp rise and decline from peak luminosity observed in the bottom panel of Fig. 6. We also note that SN2013hx and SN2008es have a similar decline from 20 to 50 days. The width of the main peak in PS15br is also not comparable to that of the SLSN IIn SN2006gy (Smith et al. 2007), as the latter has a much wider light curve of $\sim 110$ days (see bottom panel of Fig. 6). Furthermore, the PS15br peak is strongly asymmetric (see Fig. 6, Fig. 7 \& Table 3), a detail conveying against a strong interaction with a hydrogen-rich massive shell typical of IIn (and SLSN IIn) which would have resulted in a wide, almost symmetric light curve peak. The slower decline experienced by PS15br is comparable to that of the bright type II SN2009kf and could be the consequence of a more massive hydrogen layer - and hence higher contribution from hydrogen recombination - than those of the other SLSNe II. Alternatively it could be due to interaction in a similar manner to what has been observed in weakly or mildly interacting type II such as SNe 2007pk (Pritchard et al. 2012; Inserra et al. 2013a), PTF11iqb (Smith et al. 2015) and 2013fc (Kangas et al. 2016). These SNe can be explained with an interaction, weaker than of SNe IIn, with a dense CSM most likely caused by wind acceleration (Morozova et al. 2017; Moriya et al. 2017), which affects spectra and light curve evolution in a less dramatic way than what observed in SN IIn/SLSN IIn such as SN2006gy.

\subsection{Spectroscopic resemblance to other SLSNe and type II SNe}

In the top panel of Fig. 8 the comparison of the spectra around $+20 \mathrm{~d}$ post maximum with those of the other SLSNe such as SN2008es, SN2011ke and CSS121015 highlights a difference in the line evolution between SLSNe II and both a prototypical (SN2011ke) and peculiar (CSS121015) SLSN I. CSS121015 shows weaker H than SN2013hx and SN2008es with an equivalent width $(\mathrm{EW}) \mathrm{EW}(\mathrm{H} \alpha)_{\mathrm{SN} 2013 \mathrm{hx}, \text { SN2008es }}$ $10 \times \mathrm{EW}(\mathrm{H} \alpha)_{\mathrm{CSS} 121015}$. CSS121015 also exhibits Fe II lines which are more prominent than the type II SLSNe at similar phase. Indeed the Fe absorption lines in CSS121015 are stronger than those of SLSNe II SN2013hx, PS15br and $\mathrm{SN2008}$ es with $\mathrm{EW}(\mathrm{Fe})_{\mathrm{CSS} 121015} \approx \mathrm{EW}(\mathrm{Fe})_{\mathrm{SLSNeI}} \sim 5-10 \times$ $\mathrm{EW}(\mathrm{Fe})_{\text {SLSNeII }}$. Both SN2013hx and PS15br are also different from typical SLSNe I spectra. These show the same metal lines observed in CSS121015 at similar epochs. The difference between SLSNe I, dominated by broad metal lines, and SLSNe II (SN2008es, SN2013hx, PS15br), exhibiting H lines with weaker metal lines with respect to the SLSNe I, is highlighted in the top panel of Fig. 8. To investigate if SLSNe II spectroscopically behave in a similar fashion of what observed for SLSNe I (Pastorello et al. 2010; In- 

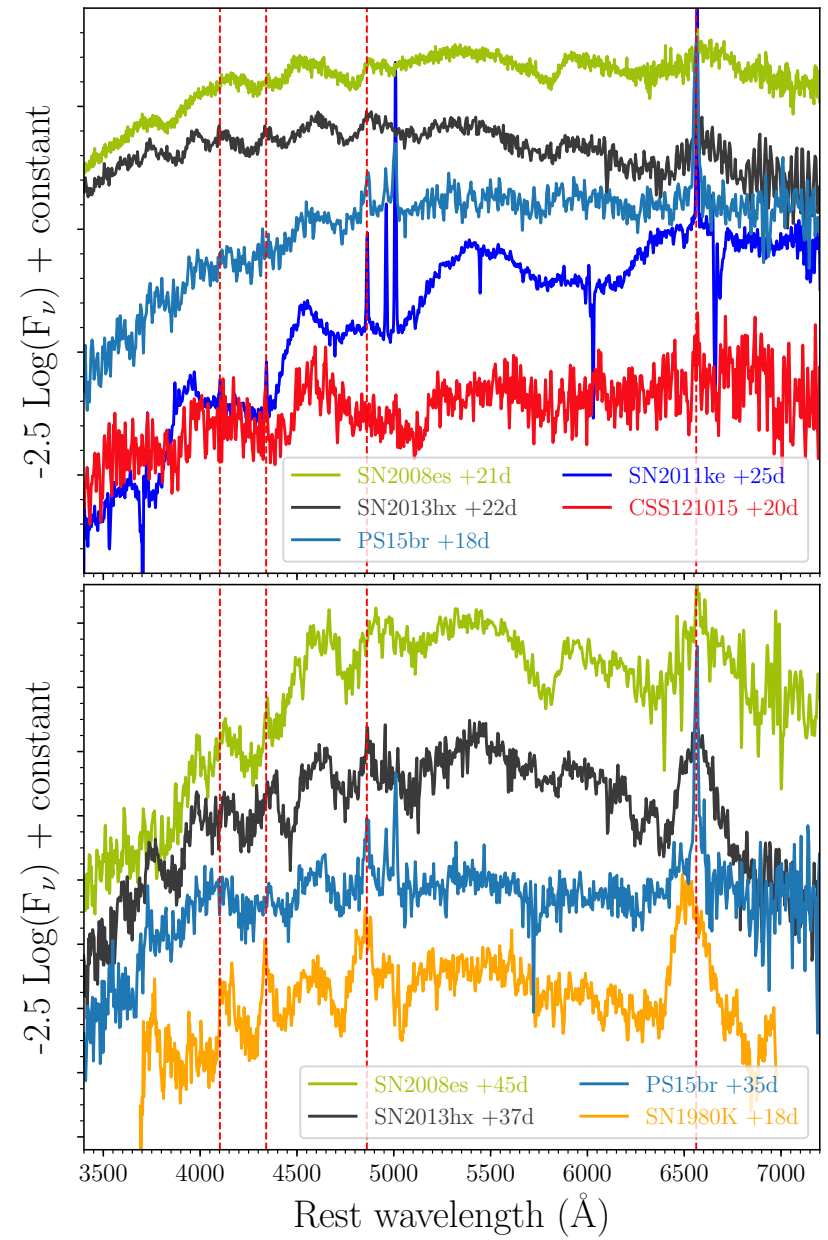

Figure 8. Top: comparison in $\log \left(\mathrm{F}_{v}\right)$ space, which highlights spectral features, of SN2013hx and PS15br spectra at $~ 20$ days from maximum with those of SLSNe showing Hydrogen in their spectra SN2008es (Miller et al. 2009) and CSS121015 (Benetti et al. 2014), together with SLSN I SN2011ke (Inserra et al. 2013b). Bottom: Comparison of the spectra of SN2013hx and PS15br at $\sim 40$ days from maximum together with that of SN2008es at similar epoch and that of the type II SN1980K at $~ 20$ day after maximum light. The spectra of the three SLSNe II are similar to that of the type II in a similar fashion to SLSNe I at 30d resembling type Ic at maximum. $\mathrm{H} \alpha$ to $\mathrm{H} \delta$ are marked with red vertical dashed lines in all panels.

serra et al. 2013b) in Fig. 8 (bottom panel) we compare the spectra of SN2013hx and PS15br with that of SN2008es at around 40 days, together with that of the type II SN $1980 \mathrm{~K}^{5}$ at 20 days past maximum light. There is an overall resemblance between SLSNe II and SN1980K. At these epochs SNe II/IIL exhibit $v \sim 8200 \mathrm{~km} \mathrm{~s}^{-1}$ components in $\mathrm{H} \alpha$ while SN2013hx shows $v \sim 8000 \mathrm{~km} \mathrm{~s}^{-1}$. Similar trends are visible for $\mathrm{H} \beta$, with $v(\mathrm{H} \beta)_{\mathrm{SN} 1980 \mathrm{~K}} \sim 7900 \mathrm{~km} \mathrm{~s}^{-1}$, which is similar to $v(\mathrm{H} \beta)_{\mathrm{SN} 2013 \mathrm{hx}} \sim 7000 \mathrm{~km} \mathrm{~s}^{-1}$. In addition, the strength of the He I $\lambda 5876$ line is similar between the objects $\left(\mathrm{EW}_{\mathrm{SN} 1980 \mathrm{~K}}=10 \AA\right.$ compared to $\mathrm{EW}_{\mathrm{SN} 2013 \mathrm{hx}}=11 \AA$, while

5 Some of such spectra were digitalised and flux calibrated in Benetti PhD thesis, 1992.
$\mathrm{EW}_{\mathrm{SN} 2008 \mathrm{es}}=18 \AA$ ). This also holds true for the Fe II feature, which has $\mathrm{EW}_{\mathrm{SN} 1980 \mathrm{~K}}=16 \AA$ and $\mathrm{EW}_{\mathrm{SN} 2013 \mathrm{hx}}=18 \AA$. Blueward of $\mathrm{H} \beta$ the profiles begin to differ, mainly due to Fe I/Ti II shown around $4000 \AA$ in SLSNe and a stronger Ca H\&K $\left(\mathrm{EW}_{\text {SLSNeII }} \sim 2-3 \times \mathrm{EW}_{\mathrm{SN} 1980 \mathrm{~K}}\right)$ The line profiles of PS15br are similar to those of SN2013hx and SN2008es, with the only exception of the $\mathrm{H} \alpha$ profile that does not show an absorption component and a rather weak He I. This support the theory that a certain degree of weak/mild interaction is at play in PS15br. PS15br does not show a blue pseudo-continuum, which would result in a rise of the flux bluewards than $\sim 5400 \AA$ (e.g. Turatto et al. 2000; Benetti et al. 2014; Inserra et al. 2016a) that characterises certain types of IIn spectra or the blue spectra with only lorentian Balmer emission line typical of SLSNe IIn such as SN2006gy (Smith et al. 2007). Indeed, bluewards than $\mathrm{H} \alpha$, PS15br is similar to SN2013hx and SN2008es in line strength.

\subsection{Velocity and temperature evolution}

In the left panel of Fig. 9 the evolution of the temperature is plotted in logaritmic space. It is derived from the blackbody fit to the continuum of our rest-frame spectra (big symbols) and colour temperatures (small symbols). Only SN2013hx has multi-band coverage before peak allowing us to estimate the temperature pre-peak (17000 to $15000 \mathrm{~K}, \sim 4.2 \mathrm{dex})$. SN2013hx shows a monotonic decline from $14000 \mathrm{~K}(4.15$ dex) at peak to $6600 \mathrm{~K}$ (3.82 dex) at 40 days, while PS15br declines from $12000 \mathrm{~K}$ (4.08 dex) to $6000 \mathrm{~K}$ (3.78 dex) showing similar temperatures to SN2013hx and SN2008es in the same time coverage. After 50 days, the temperatures of the objects flatten at $\sim 5500 \mathrm{~K}$ (3.74 dex). SN2008es shows a similar temperature evolution to that of SN2013hx. The overall temperature evolution of SN2013hx, PS15br and SN2008es is similar to those observed in SLSNe I and normal type II $\mathrm{SNe}$ with a similar decline to those of type II. We note that in the first 10 days after maximum PS15br shows a drop in temperature similar to those of type II and SN2013hx, but $\sim 0.1$ dex fainter than the latter.

In the right panel of Fig. 9 the expansion velocities for our SLSNe, as inferred from the position of the absorption minima of the P-Cygni profiles for $\mathrm{H} \alpha$ and Fe II (average of $\lambda \lambda 4924,5018,5169)$ are compared with those of the only other SLSN II SN2008es, an average of SLSNe I and type II SNe. The uncertainties were estimated from the scatter between several measurements. In the case of $\mathrm{H} \alpha$, the velocities were computed from the FWHM of the emission lines for PS15br and for SN2008es in spectra after 40 days. $\mathrm{H} \alpha$ velocities decline monotonically from $12000 \mathrm{~km} \mathrm{~s}^{-1}$ to 7500 $\mathrm{km} \mathrm{s}^{-1}$ on the time scale available for SN2013hx. An almost identical decrease is observed in SN2008es and PS15br in the available period $(20<$ phase $($ day $)<45)$ even if PS15br values are due to the broad emission component. The decline rate is qualitatively similar to normal luminosity type II SNe which have average velocities that are lower by about $1200 \mathrm{~km} \mathrm{~s}^{-1}$ at similar epochs, whereas SN1980K H $\alpha$ velocity evolution resembles those of SLSNe II. The velocities measured from the full width at half maximum (FWHM) of the emission components appear to be systematically higher than those inferred from the minima. The Fe II velocities are usually assumed to be better tracers of the photospheric velocity and they are similar in SN2013hx, SN2008es and 

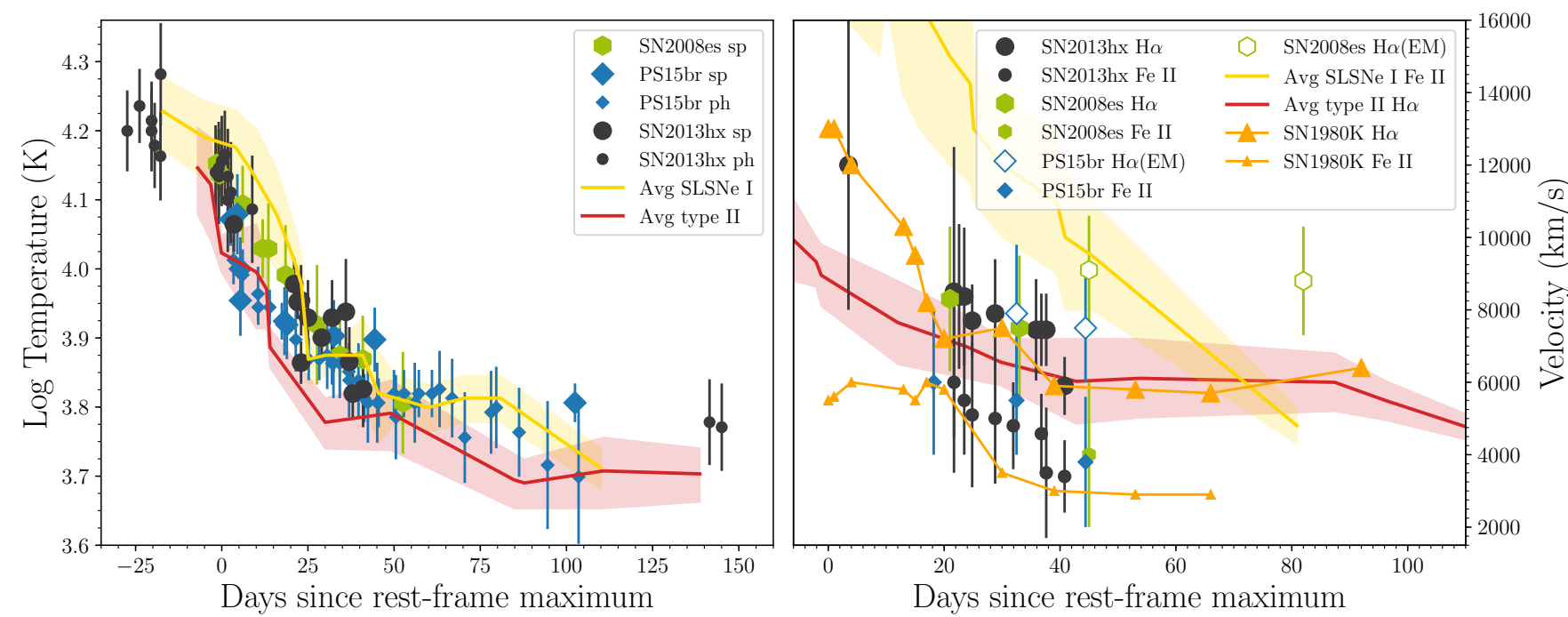

Figure 9. Left: SN2013hx (black) and PS15br (blue) temperature evolution compared with that of SN2008es (green). The gold region represent the $99.73 \%$ region of the temperature space of SLSNe I (Inserra et al. 2013b; Nicholl et al. 2015), while the red that of a type II collection (Elmhamdi et al. 2003; Maguire et al. 2010; Inserra et al. 2011, 2012a; Botticella et al. 2010; Tomasella et al. 2013; Gutiérrez et al. 2017b). Right: $\mathrm{H} \alpha$ and Fe II evolution of SN2013hx (black) and PS15br (blue) compared with that of SN2008es (green), the type II SN1980K (orange triangle and line for $\mathrm{H} \alpha$ and smaller symbols for Fe II; Barbon et al. 1982). The gold region represents the velocity space of Fe II line in SLSNe I, while the red that of $\mathrm{H} \alpha$ in type II SNe. Open symbols refer to velocities computed with the FWHM of the emission lines.

PS15br decreasing from $6000 \mathrm{~km} \mathrm{~s}^{-1}$ to $4000 \mathrm{~km} \mathrm{~s}^{-1}$ (we note that our measurements are in agreement with those presented by Nicholl et al. 2015). These show the same decline as displayed in normal faster-declining type II, but with a shift or delay by of $\sim 15-20$ days. SLSNe II Fe II velocities are quite different from those of SLSNe I, in that they are $\sim 7000 \mathrm{~km} \mathrm{~s}^{-1}$ slower.

\section{LATE TIME SPECTRA AND SIGNS OF INTERACTION IN SLSNE II}

In Fig. 10 we show the late VLT+FORS2 spectrum of SN2013hx and those of PS15br obtained with VLT+Xshooter and NTT+EFOSC2, which are the latest spectra and the only at late ( $>150$ d from peak) phase for SLSNe II obtained to date. For SN2013hx, a lower signalto-noise spectrum was taken by PESSTO on $+210 \mathrm{~d}$, and is also shown in Fig. 10. This NTT spectrum does not appear to be significantly different to the FORS2 $+237 \mathrm{~d}$ spectrum and as it is much lower quality, we will not discuss it further. In the case of PS15br, PESSTO spectra do not show any substantial evolution with respect to those of XShooter and have lower $\mathrm{S} / \mathrm{N}$, hence we will focus our analysis only on the XShooter spectra.

There is no distinguishable line at the position of [O I] $\lambda \lambda 6300,6364 \AA$, which is usually prominent in core-collapse supernovae. There is perhaps some hint of weak and broad emission in SN2013hx that could be attributed to this line, but the the detection is $\lesssim 2 \sigma$ with respect to the continuum flux and is not convincing (see Fig. 11). In Figure 11 we compared our SN2013hx spectrum with those of the fastdeclining type II (or type IIL) SN1980K and the broadline type Ic SN1998bw at late epochs and scaled to the same luminosity distance of SN2013hx $(570.3 \mathrm{Mpc})$ in order to match the rest-frame spectral luminosity exhibited by SN2013hx. The spectra of SN2013hx and SN1980K are similar, although they differ by 135 days with respect to the peak epochs, and as consequence also similar to that of PS15br shown in the bottom plot. The overall similarity with type IIL SNe suggest that also SLSNe II are consistent with a star explosion similar in mass to those of normal core-collapse SNe. The $\mathrm{H} \alpha$ profile and the blueward features are also different from that of iPTF13ehe, a SLSN I with late-time interaction with a hydrogen shell. The width of the central and blue components of SN2013hx $\mathrm{H} \alpha$ are comparable with those of PS15br at $\sim 337$ d. PS15br $\mathrm{H}_{\beta}$ emission component is stronger than those almost non-existent of SN2013hx and SN1980K.

The $+247 \mathrm{~d}$ SN2013hx spectrum and all the late PS15br spectra are dominated by a strong and multicomponent $\mathrm{H} \alpha$ profile, and the simplest explanation would be that the SN ejecta is interacting with an H-rich, highly asymmetric CSM (as previously suggested for similar late time interactions; e.g. Benetti et al. 2016). There are no other lines visible in the optical with the exception of $\mathrm{H} \beta$, and the continuum is faint. However, as shown in Fig. 10 (bottom panel), PS15br NIR spectra exhibit Paschen lines in emission, as well as He I $\lambda 10830$ that obscures $\mathrm{Pa} \gamma$. We note that both $\mathrm{H} \beta$ and $\mathrm{Pa} \alpha$ profiles resemble that of $\mathrm{H} \alpha$ with a prominent blue shoulder. In SN2013hx the $\mathrm{H} \beta$ flux is $\sim 50$ times less than that of the $\mathrm{H} \alpha$ profile and $\sim 30$ times less than $\mathrm{H} \alpha$ central component (see Table 4). For case B recombination in the temperature regime $2500 \leq \mathrm{T}(\mathrm{K}) \leq 10000$ and electron density $10^{2} \leq$ $n_{e} \leq 10^{6}$ the $\mathrm{H} \alpha$ line should be 3 times stronger than $\mathrm{H} \beta$. However, the case $\mathrm{B}$ recombination is never observed in type II before a couple of years since both the $n=4 \rightarrow n=2(\mathrm{H} \beta)$ 


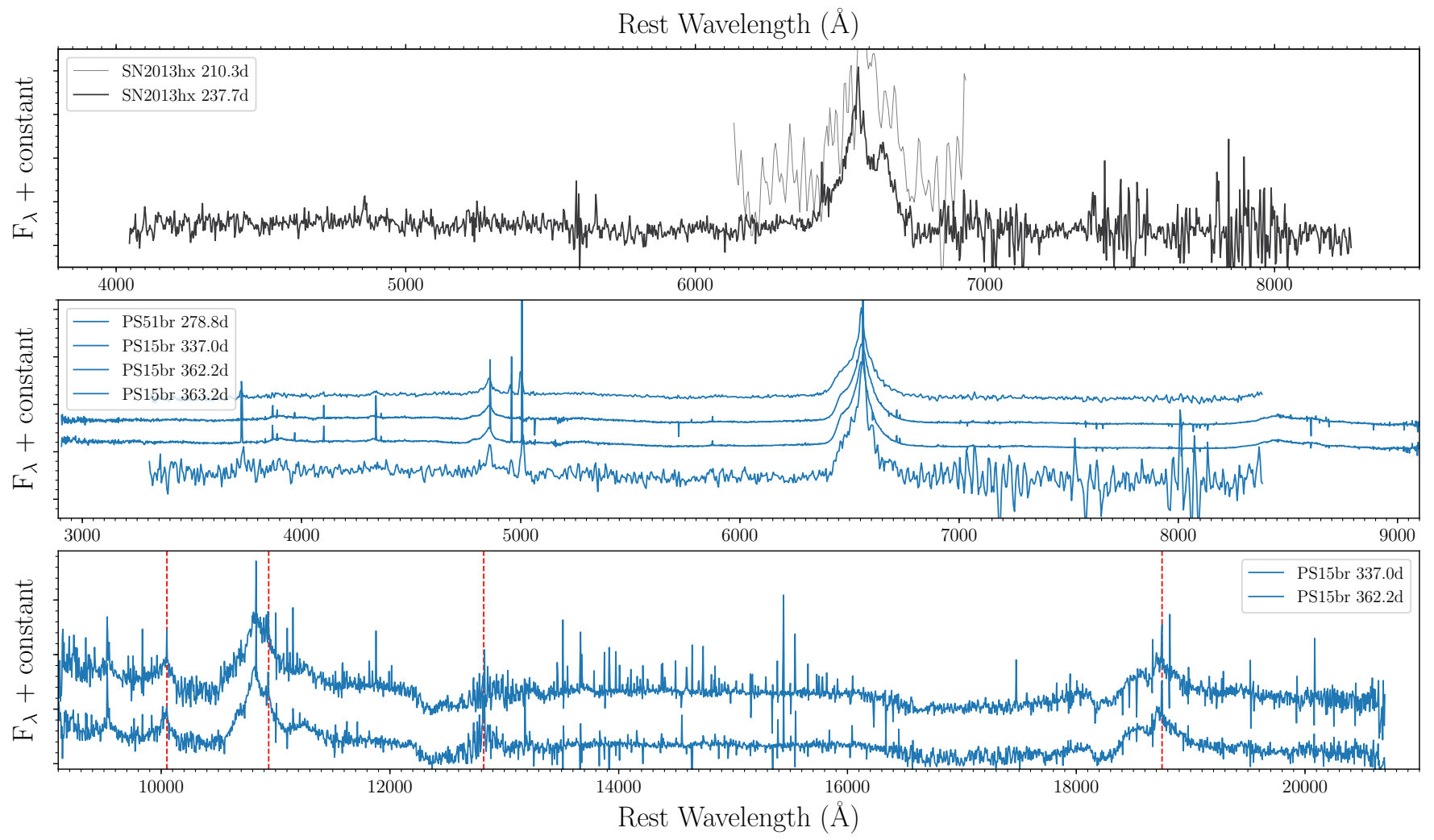

Figure 10. Top: Last two spectra of SN2013hx. Middle: Late time optical spectra of PS15br. Bottom: Late time NIR spectra of PS15br. Paschen lines from $\alpha$ to $\delta$ are identified by vertical dashed red lines.

and $n=3 \rightarrow n=2(\mathrm{H} \alpha)$ transitions are optically thick (Xu et al. 1992). In this case the $\mathrm{P} \alpha(n=4 \rightarrow n=3)$ transition will depopulate the $n=4$ state, with the photons escaping to produce the $\mathrm{P} \alpha$ emission line. $\mathrm{H} \alpha$ however is produced strongly as the only other alternative for depopulation of the the $n=3$ state is Lyman $\beta$. In PS15br the $\mathrm{H} \beta$ flux is $\sim 10$ times less than that of the $\mathrm{H} \alpha$ profile and as expected the $\mathrm{P} \alpha$ emission line is visible. The integrated luminosity of the overall profile of $\mathrm{H} \alpha$ line are $\sim 9$ and $\sim 20$ times those of a normal type II at similar phase (Kozma \& Fransson 1998) for SN2013hx and PS15br, respectively. This suggests an additional contribution to $\mathrm{H} \alpha$ luminosity in both cases. What powers the decline rate of the late time light curve is an open question. One could speculate that the signature of interaction we see at this late phase is indicative of the same powering source for the light curves between 100-260 days. Late interaction has been observed in bright type IIL such as SNe 1979C, 1980K and 1986E (Fesen et al. 1999; Cappellaro et al. 1995; Benetti et al. 2016, and references therein).

The SN2013hx $\mathrm{H} \alpha$ profile in Fig. 12 has a triple peak structure spanning $7400 \mathrm{~km} \mathrm{~s}^{-1}$ across the base (cfr. Tab 4), which is similar to the triple peak shown by the interacting type II PTF11iqb after 500 days (Smith et al. 2015). The central component is consistent with having zero rest-frame velocity, and the blue component peaks at $-4700 \mathrm{~km} \mathrm{~s}^{-1}$ with respect to this $\mathrm{H} \alpha$ rest-frame velocity. This blue component appears to be the weakest of the three. The red component is at $+4000 \mathrm{~km} \mathrm{~s}^{-1}$. We analysed the feature with gaussian, lorentzian and pseudo voigt line profiles having set a priori the number of components (three) but letting vary the width and intensity of each component. We retrieved the best fit with three voigt profiles with widths of $\sim 3400 \mathrm{~km} \mathrm{~s}^{-1}$. However, gaussians have a similar goodness of fit and produce similar line widths but somewhat different line fluxes. We performed the same analysis on the 337 days spectrum of PS15br. In this case, $\mathrm{H} \alpha$ shows a two components profile, a blue at $-4700 \mathrm{~km} \mathrm{~s}^{-1}$ w.r.t. $\mathrm{H} \alpha$ and the central at almost zero rest-frame velocity $\left(-390 \mathrm{~km} \mathrm{~s}^{-1}\right)$. This profile is similar to that shown by SN2013hx, with the exclusion of the red component, and it is also shown in the $\mathrm{Pa} \alpha$ (see middle panel of Fig. 13) but not in Pay, which is blended with the more prominent He I $\lambda 10830$. The best fit for these components is retrieved with voigt profiles of width $\sim 3100$ and $\sim 4800$ $\mathrm{km} \mathrm{s}^{-1}$ for the blue and central component, respectively (see Fig. 13).

In general, the line profile could be schematically explained by:

(i) a relatively broad emission from the $\mathrm{SN}$ ejecta with FWHM $\sim 3400 \mathrm{~km} \mathrm{~s}^{-1}$;

(ii) interaction of the H-rich ejecta with:

(a) a ring/disk edge-on,

(b) a dense, clumpy CSM;

(iii) a likely unshocked CSM that could explain the narrow, unresolved emission on the top of the central component.

The blue wing of the $\mathrm{H} \alpha$ emission of SN2013hx and 

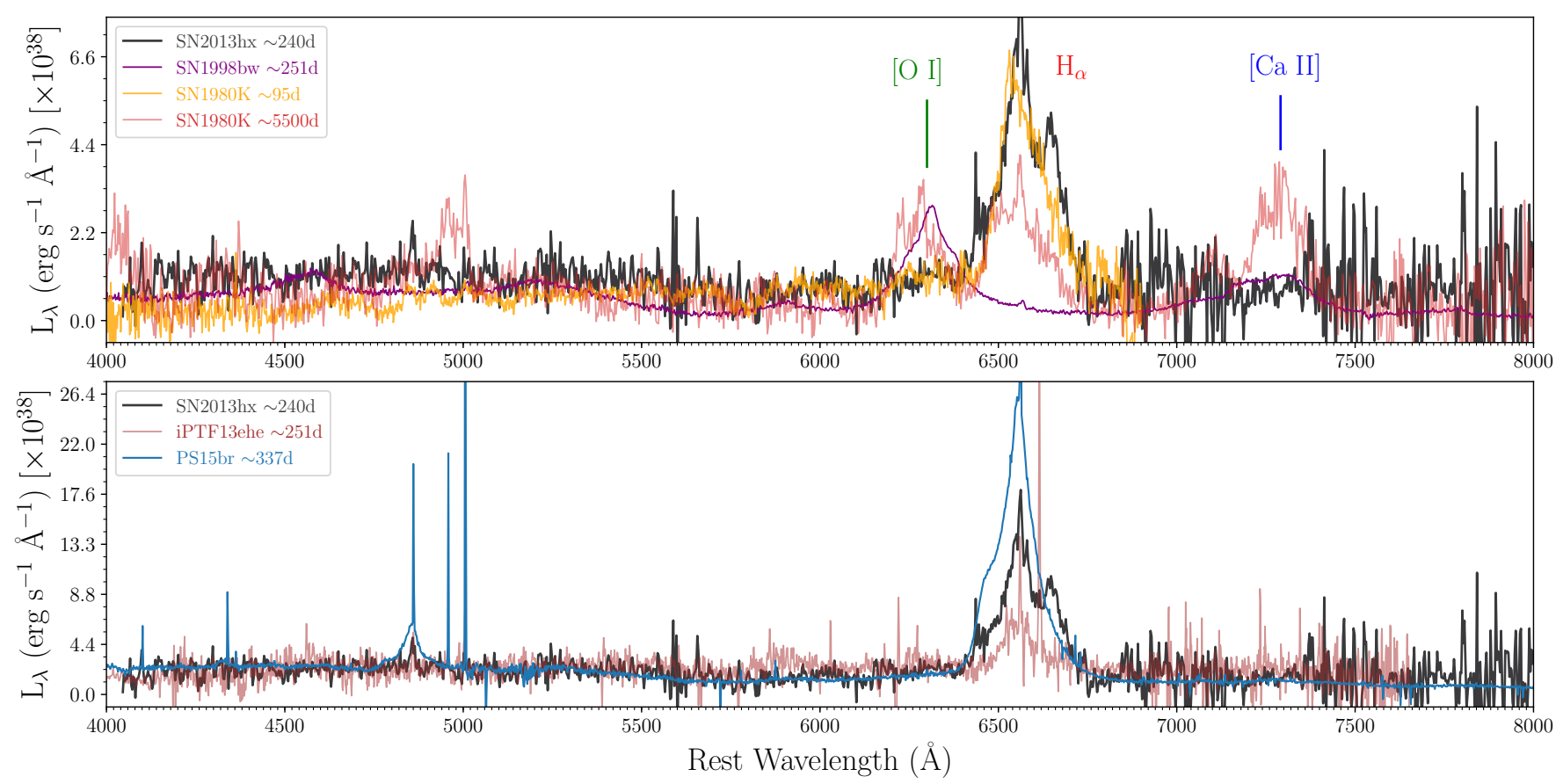

Figure 11. Top: the VLT+FORS2 spectrum of SN2013hx compared with late spectra of the type IIL SN1980K (Barbon et al. 1982) and a late spectrum of the broad-line type Ic SN1998bw (Patat et al. 2001). All the spectra have been scaled to the continuum flux level of SN2013hx. The most prominent line of nebular core-collapse spectra are labelled. Bottom: the VLT+FORS2 spectrum of SN2013hx and the VLT+XShooter (337d) compared with SLSNe I iPTF13ehe, a slow SLSN I showing late H $\alpha$ emission.

Table 4. Late $\mathrm{H} \alpha$ profile measurements of SLSNe II SN2013hx ( 240 day) and PS15br ( 337 day)

\begin{tabular}{lccccc}
\hline \hline Feature & $\begin{array}{c}\text { Peak } \\
(\AA)\end{array}$ & $\begin{array}{c}\text { Width } \\
(\AA)\end{array}$ & $\begin{array}{c}\text { Width } \\
\left(\mathrm{km} \mathrm{s}^{-1}\right)\end{array}$ & $\begin{array}{c}\text { Velocity w.r.t. H } \alpha \text { peak } \\
\left(\mathrm{km} \mathrm{s}^{-1}\right)\end{array}$ & $\begin{array}{c}\text { Flux } \\
\left(\mathrm{erg} \mathrm{s}^{-1} \mathrm{~cm}^{-2}\right)\end{array}$ \\
\hline \multicolumn{5}{c}{ SN2013hx } \\
\hline Blue & 6459.8 & 73.8 & 3427 & -4700 & $1.1 \times 10^{-16}$ \\
Central & 6558.9 & 73.8 & 3376 & -190 & $4.0 \times 10^{-16}$ \\
Red & 6650.8 & 73.8 & 3329 & 4000 & $2.2 \times 10^{-16}$ \\
\hline \multicolumn{7}{c}{ PS15br } \\
\hline Blue & 6460.7 & 66.8 & 3102 & -4700 & $2.4 \times 10^{-16}$ \\
Central & 6556.5 & 105.2 & 4813 & -320 & $9.3 \times 10^{-16}$ \\
\hline
\end{tabular}

PS15br roughly coincides in wavelength with the corresponding blue wing of the $\mathrm{H} \alpha$ profile at 37 and 102 days as shown by the top panel of Figs. $12 \& 13$. If the absorption minimum maintains the same velocity shown at early time, it will be at a similar wavelength to the peak of the blue component. However, if SLSNe II evolution follows closely that of fast-declining type II as suggested by the spectroscopic evolution (see Section 3.2 and Fig. 11), we would not expect any absorption component from the $\mathrm{H} \alpha$ able to dim the blue peak. Instead an asymmetric, clumpy CSM could explain the difference in intensity between the blue and the red component in SN2013hx and the absence of a red component in PS15br. If we considered the blue component of SN2013hx to be a consequence of a boxy profile, and hence more a shoulder than a peak, we would still need to invoke an asymmetric configuration. Such CSM structure would have a two-component wind with a spherically symmetric region responsible for the boxy profile and the presence of a denser clump in the direction opposite to that of the observer in order to explain the intensity of the red component in SN2013hx. Hence both the scenarios would suggest an asymmetric configuration of the CSM. Such configurations have been invoked to explain late time multicomponent $\mathrm{H} \alpha$ profiles for several type II SNe (e.g. Uomoto \& Kirshner 1986; Fesen et al. 1999; Pozzo et al. 2004; Inserra et al. 2011; Benetti et al. 2016). 


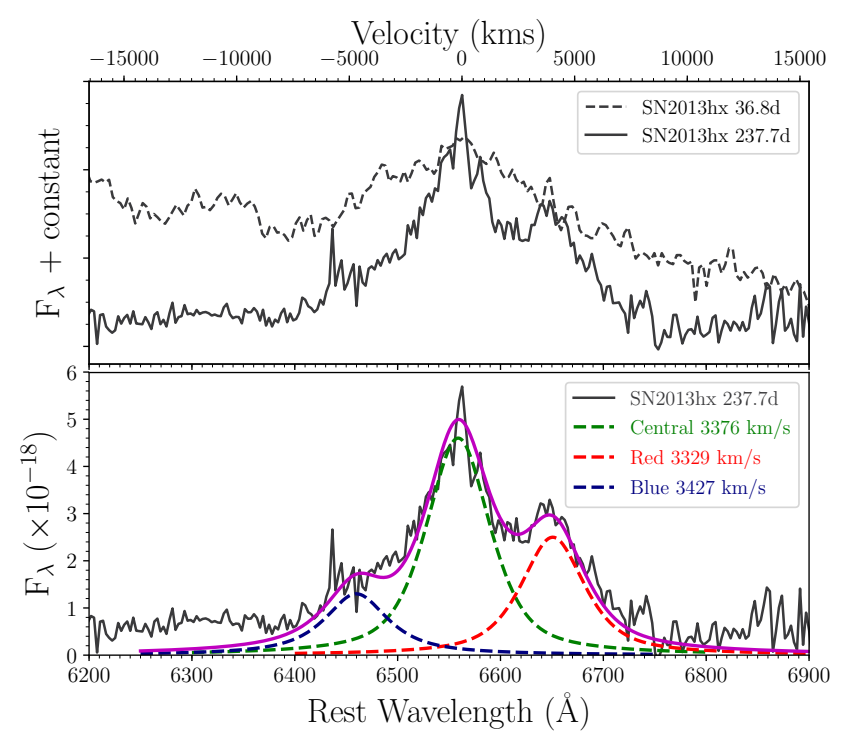

Figure 12. Top: $\mathrm{H} \alpha$ multicomponent profile of SN2013hx spectrum at 238 days (black solid line) with that exhibited at 37 days (black dashed line) scaled in flux to match the previous spectrum. Bottom: $\mathrm{H} \alpha$ multicomponent profile of the spectrum at 238 days since maximum. The spectrum is dominated by interaction with a H-rich CSM.

\section{DISCUSSION}

SN2013hx and PS15br provide, for the first time, a small sample of SLSNe II useful for the understanding of the hydrogen-rich SLSNe. These SLSNe are different than those undergoing strong interaction with an optically thick CSM showing spectra with strong optical lines having multiple emission components such as SN2006gy (Smith et al. 2007). They have luminosity spanning from $-20>\mathrm{M}_{g}>-22$, similar spectral evolution and an origin in faint host galaxies. The latter is surprisingly similar to the characteristic locations of their hydrogen-free counterparts (Lunnan et al. 2014; Leloudas et al. 2015a; Chen et al. 2015). Their photospheric $(<100 \mathrm{~d})$ spectroscopic evolution resembles those of bright type II (or type IIL) SNe with a delay of 15-20 days and their light curves show a linear decline over 100 days post peak. Hence they are more luminous and evolve slower than normal type IIL SNe. These two characteristics (more luminous, slower evolution) also link SLSNe I to normal type Ic SNe in a similar way. Therefore, it might be that the power source responsible for the differences between SLSNe I and SNe Ic is also accountable for those between SLSNe II and SNe II.

The extreme luminosity of SLSNe I has been explained with a few scenarios and we use our dataset to constrain similar models for the origin of SLSNe II. The light curves that we will use in the following Sections are the full, estimated, bolometric light curves that have been corrected for flux missed in both UV and NIR as described in Section 4.2.

\section{$6.1 \quad{ }^{56} \mathrm{Ni}$ driven explosion}

To test if radioactive heating could be the main source of energy, we can assume that $\gamma$-rays from ${ }^{56} \mathrm{Co}$ decay are fully

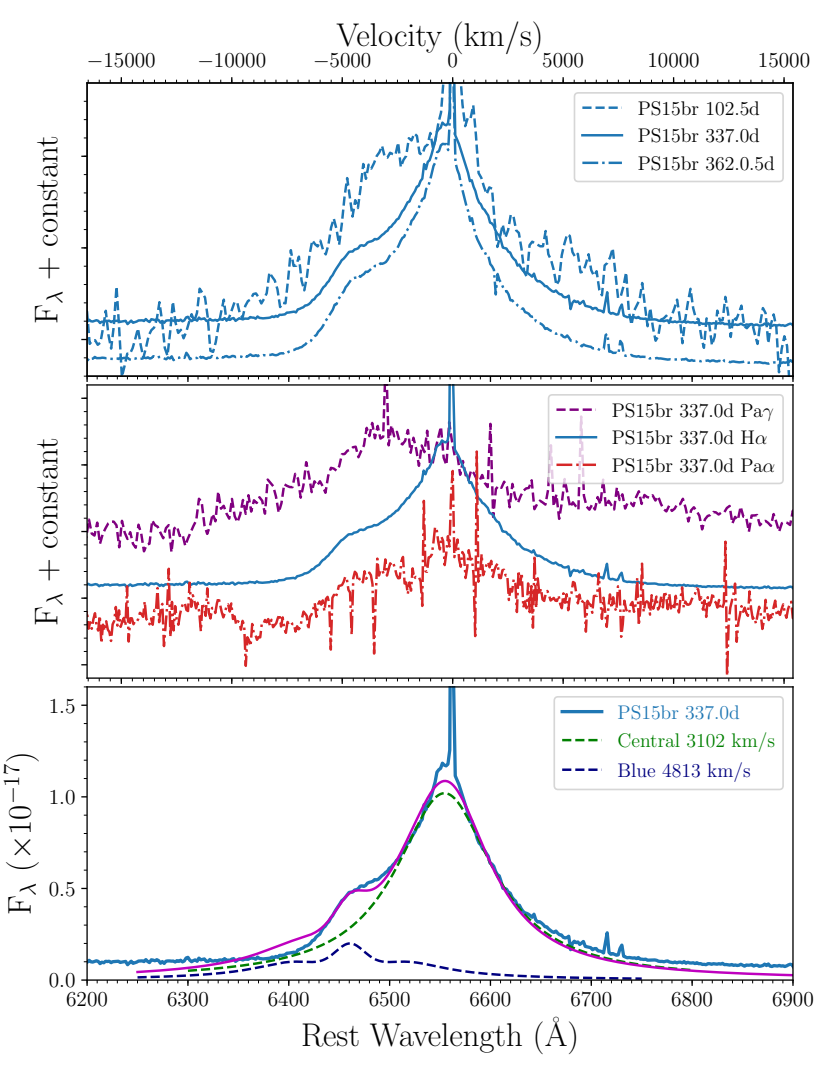

Figure 13. Top: $\mathrm{H} \alpha$ multicomponent profile of PS15br spectrum at 337 and 362 days (blue lines) with that exhibited at 102 days (cyan line) scaled in flux to match the previous spectrum. Middle: $\mathrm{H} \alpha$ (blue line), $\mathrm{Pa} \gamma$ (magenta line) and $\mathrm{Pa} \alpha$ (red line) multicomponent profiles of the PS15br spectrum at 337 days. Bottom: $\mathrm{H} \alpha$ multicomponent profile of the PS15br spectrum at 337 days since maximum. The spectrum is dominated by interaction with a $\mathrm{H}-$ rich CSM.

thermalized and can hence estimate the ${ }^{56} \mathrm{Ni}$ mass needed for these events comparing their bolometric luminosity with that of SN1987A. For SN2013hx, the peak would require a ${ }^{56} \mathrm{Ni}$ mass of $M\left({ }^{56} \mathrm{Ni}\right) \sim 8.0 \mathrm{M}_{\odot}$ and the luminosity in the tail phase at around 140 days would be $M\left({ }^{56} \mathrm{Ni}\right) \sim 1.0 \mathrm{M}_{\odot}$. For PS15br, the luminosity at 100 days would require $M\left({ }^{56} \mathrm{Ni}\right) \sim$ $2.2 \mathrm{M}_{\odot}$. Although these objects are $\mathrm{H}$-rich and hence hydrogen recombination should play some role in the photospheric phase of the light curve, we applied our toy model (see Appendix D of Inserra et al. 2013b) to have an alternative estimate of the ${ }^{56} \mathrm{Ni}$ mass required to power the late light curves. We retrieved values of $4.3<M\left({ }^{56} \mathrm{Ni}\right)<8.4 \mathrm{M}_{\odot}$ for $\mathrm{SN} 2013 \mathrm{hx}$ (depending on whether or not we included the tail phase data after 142 days) and $M\left({ }^{56} \mathrm{Ni}\right) \sim 4.4 \mathrm{M}_{\odot}$ for PS15br, for which we do exclude the tail since our first late spectrum showing interaction is coincident with the first photometric point of the tail.

Although there is some uncertainty in these numbers, the large ${ }^{56} \mathrm{Ni}$ masses could suggest a pair instability explosion (Barkat et al. 1967; Fraley 1968). However, due to the large ejecta masses expelled in pair instability explosions, the rise times of the light curves is a factor $2-3$ greater than that observed in our objects (Woosley et al. 2007). In case of pair instability, the ejecta masses should be $2-5$ 

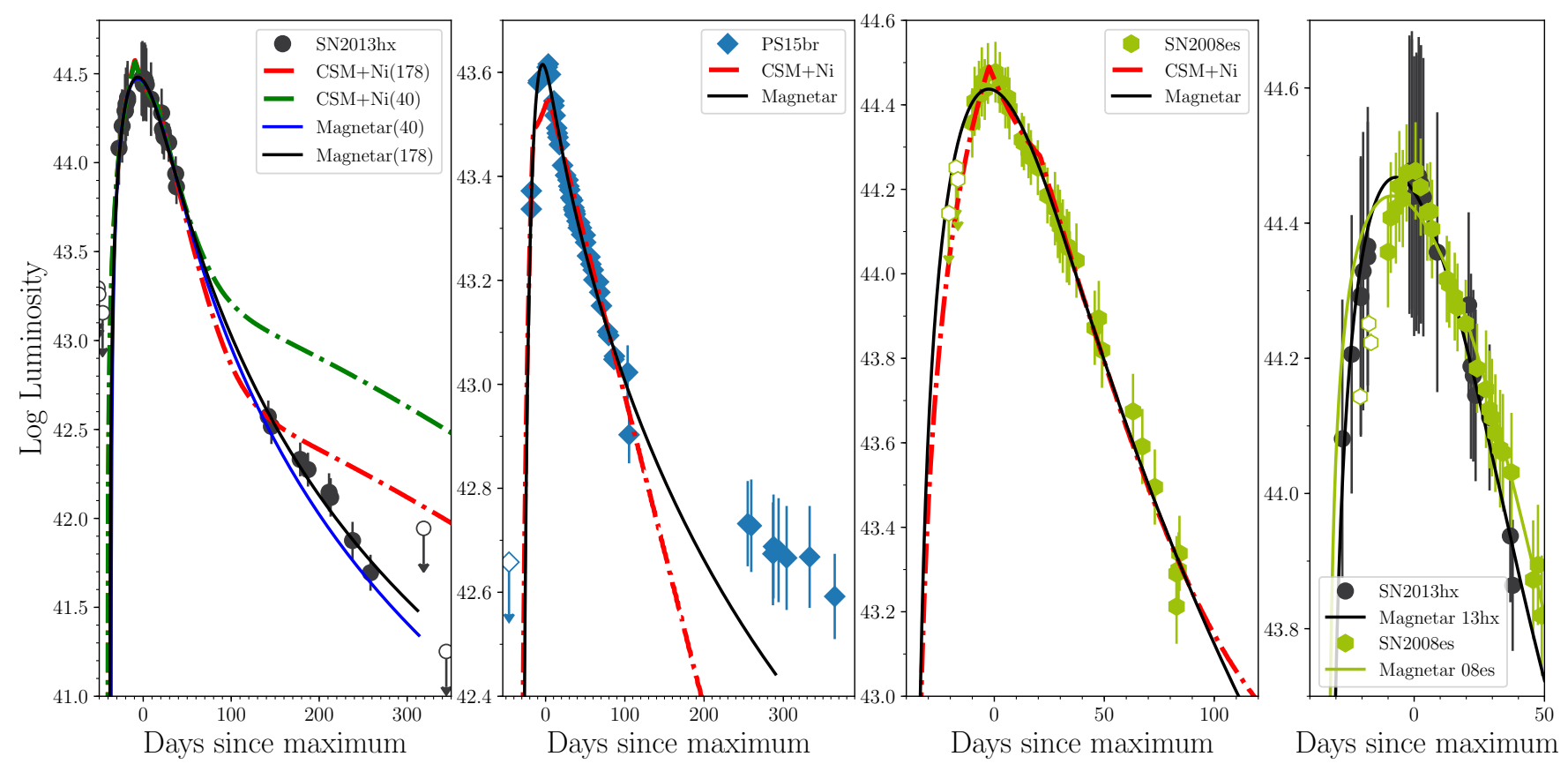

Figure 14. Bolometric light curves of SN2013hx, PS15br and SN2008es compared with the best-fit models. The black solid line (and also the blue for SN2013hx) refers to the magnetar model, while the red dot-dashed (and also the green for SN2013hx) refers to the $\mathrm{CSM}+\mathrm{Ni}$ model. Limits are shown as empty symbols. For the case of SN2013hx, the (40) models consider up to 40 days past maximum, while the (178) refer to the models considering up to 178 days. the plot on the far right shows a comparison of bolometric light curves and magnetar fit around maximum light for SN2013hx and SN2008es. This highlights how the difference time baseline coverage gives slightly different magnetar parameters (see Table 6)

times greater than the ${ }^{56} \mathrm{Ni}$ mass (Umeda \& Nomoto 2008) and such amount of material would be mainly composed of $\mathrm{He}\left(>13 \mathrm{M}_{\odot}\right)$ and $\mathrm{H}\left(>11 \mathrm{M}_{\odot}\right)$ leading to a long plateau in their light curves (Woosley \& Weaver 1995). This has not been observed, implying that the late-time evolution of the light curve of SN2013hx and PS15br is not driven by ${ }^{56} \mathrm{Ni}$ but is due to some late interaction (see Section 5).

\subsection{Circumstellar medium interaction}

In SN2013hx, PS15br and SN2008es we do not observe narrow and intermediate features suggesting an ongoing interaction with a CSM. However, we cannot exclude a priori such scenario since if the CSM is opaque and the shock encounters no further CSM at larger radii, the usual signatures of interaction spectra can be avoided (Smith \& McCray 2007; Chevalier \& Irwin 2011). This could, in principle, explain the spectra evolution in the the objects.

The dense CSM can be produced by a wind or by a shell ejected prior the SN explosion. In the former case, following the model of Blinnikov \& Bartunov (1993) used to explain type IIL, a dense stellar wind that reprocesses the UV photons produced at shock breakout can boost the luminosity up to superluminous magnitudes. That would need an increase in the mass-loss rate by one to two orders of magnitude, with respect to those observed in red supergiant (RSG) and hence reaching $\dot{M} \sim 10^{-3} \mathrm{M}_{\odot} \mathrm{yr}^{-1}$. However, in this scenario after $\sim 50-80$ days from peak, the light curve should show a drop in magnitude. The higher the peak luminosity, the greater the drop with at least a decrease of two mag in a superluminous case - and a subsequent settling onto a ${ }^{56} \mathrm{Co}$ tail. Our dataset, including SN2008es, does not show such a drop around that phase or even later.

From the blackbody fit to the SED (see Section 4.4) and the bolometric luminosity (see Section 4.2), we can estimate the peak radius of SN2013hx and PS15br to be $3 \times 10^{15} \mathrm{~cm}$ and $2.3 \times 10^{15} \mathrm{~cm}$, respectively. These radii are a factor 30 and 10 greater than that of a large RSG (Smith et al. 2001) and as a consequence the opaque CSM shell forming the envelope is not bound to the star. Following the formalism of Quimby et al. (2007); Smith \& McCray (2007), the peak luminosity produced by the radiation emitted by a shocked, thermalised shell is

$L \propto \frac{\mathrm{M}_{\mathrm{sh}} v_{\mathrm{ph}}^{2}}{2 \mathrm{t}_{\max }}$,

where $\mathrm{M}_{\mathrm{sh}}$ is the mass of the dense CSM shell, $v_{\mathrm{ph}}$ is the velocity of the pseudo-photosphere and $t_{\max }$ is the rise time. Assuming a rise time of 37 days for SN2013hx and 35 days for PS15br (cfr. Section 3.1) and a pseudo photosphere of 6000 $\mathrm{km} \mathrm{s}^{-1}$ (cfr. Section 4.4) we can infer $2.5 \mathrm{M}_{\odot}$ and $0.5 \mathrm{M}_{\odot}$ for the CSM masses of SN2013hx and PS15br, respectively.

The aforementioned radii imply a wind distance of 300 and $150 \mathrm{AU}$ for the two objects and inferring a wind velocity of $10-100 \mathrm{~km} \mathrm{~s}^{-1}$ (which brackets RSG and faster luminous blue variable wind velocities), the wind must have begun $\sim 10-100$ yr before the explosion of SN2013hx and $\sim 7-70 \mathrm{yr}$ before that of PS15br. Hence, the mass-loss rates would be 0.03-0.25 $\mathrm{M}_{\odot} \mathrm{yr}^{-1}$ and $0.007-0.07 \mathrm{M}_{\odot} \mathrm{yr}^{-1}$, which are too large to be produced by a normal steady stellar wind (Smith 
2014). Therefore if a a dense CSM scenario is invoked as the explanation of the peak luminosity, it would suggest that an unbound CSM shell configuration.

In order to reproduce the bolometric light curve of SN2013hx, PS15br and also SN2008es we use a semi-analytic code based on the equations of Chatzopoulos et al. (2012) and implemented by Nicholl et al. (2014). We use the model considering both CSM shell and radioactive decay of ${ }^{56} \mathrm{Ni}$ contribution to the light curves, setting $\delta=2$ and $n=12$ for the SN ejecta inner and outer power-law density profile slopes, respectively, in all cases. We assume Thomson scattering to be the dominant source of opacity with a solar mixture of mass fraction $(X=0.7)$, which gives an opacity $\kappa=0.2(1+X)=0.34 \mathrm{~cm}^{2} \mathrm{~g}^{-1}$ that is the standard value for a hydrogen and helium rich ejecta. An identical configuration was previously used by Chatzopoulos et al. (2013) to fit the bolometric light curve of SN2008es. Our best fits are shown in Fig. 14 (red dot-dashed line), while their parameters are reported in Table 5 . We did not fit the tail of PS15br since that is due to interaction. Since the late SN2013hx tail may be dominated by interaction with a different and probably less dense component see Section 5 - we made two illustrative fits. First, we fitted the light curve using data up to 40 days after maximum and then a second using all data out to $178 \mathrm{~d}$ (before the clear inflection in slope; see Sections $3.1 \& 4.2$ ) and before the first spectrum showing interaction with another CSM (at $210 \mathrm{~d}$, see Section 3.2$)$. We derive $2.1 \lesssim \mathrm{M}_{\mathrm{CSM}} \lesssim 2.7 \mathrm{M}_{\odot}$, $0.0 \lesssim \mathrm{M}\left({ }^{56} \mathrm{Ni}\right) \lesssim 2.0 \mathrm{M}_{\odot}$ and $9.5 \lesssim \mathrm{M}_{\mathrm{ej}} \lesssim 12.9 \mathrm{M}_{\odot}$. Following the same methodology we also estimate that a wind of 2.6 and $2.3 \mathrm{M}_{\odot}$ (or 4.7 and $3.9 \mathrm{M}_{\odot}$ in case of dense shell configuration) are responsible for the late interaction of SN2013hx and PS15br, respectively. The ${ }^{56} \mathrm{Ni}$ masses are consistent with the aforementioned limit and the CSM mass is consistent with observation of a pseudo-photosphere at the velocity reported in Section 4.4. This mass is of the order of that associated with impulsive mass ejections as discussed in Smith \& Owocki (2006). However, the ejecta masses are at least a factor two greater than what is expected to avoid an optical plateau from hydrogen recombination, while the total masses (ejecta+CSM+remnant) lie in the upper end of those observed and theorised for type II SNe (Smartt 2009; Janka 2012; Smartt 2015). Such CSM masses are more similar to those of stripped-envelope SNe and not of type II. This two component wind CSM configuration that is required to explain the late interaction of SN2013hx and PS15br, is difficult to explain with a single steady wind.

\subsection{Internal energy source}

Despite the potential of the CSM scenario to describe the main observational data - light curves and spectra evolution - and the overall picture observed in SLSNe II, there are still some details that would be easier to explain with a central engine that deposits its energy into a supernova explosion and significantly enhance the luminosity.

As observed in Section 4.2, PS15br has an asymmetric light curve may be difficult to explain with a CSM interaction, as also shown in Fig. 14. In addition SLSNe II have bell shaped light curves around peak only a factor 1.2 wider than those of SLSNe I. Furthermore, they show similar spec-

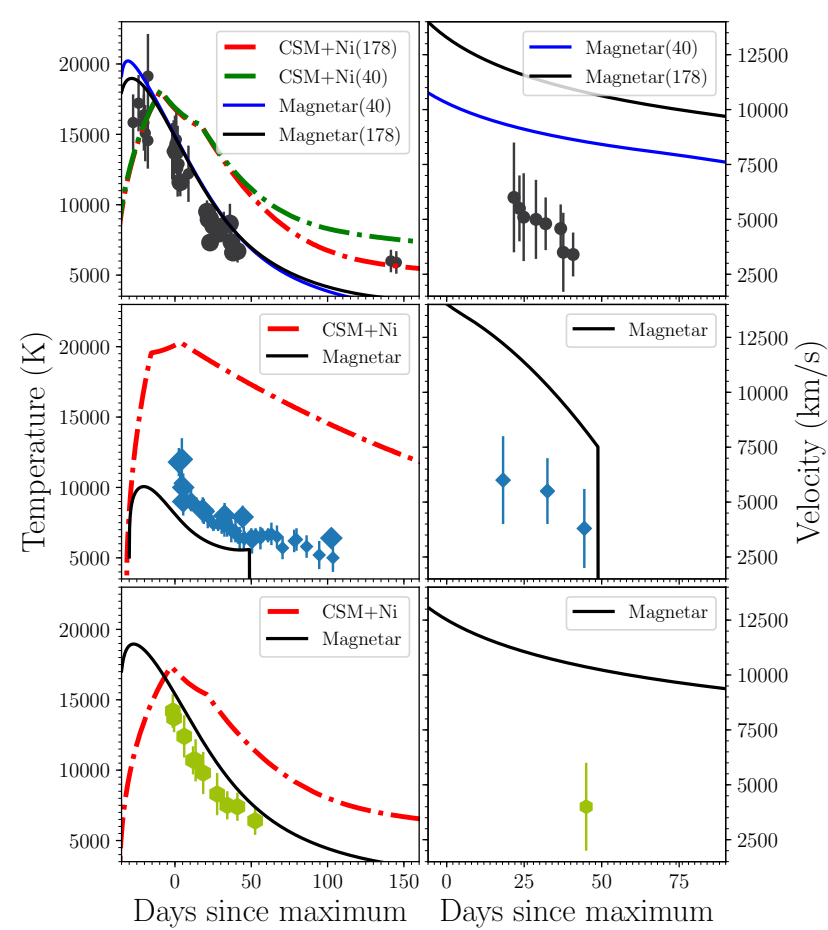

Figure 15. Temperature and velocity evolution (only for the Fe II lines tracing the photospheric evolution) of SN2013hx, PS15br and SN2008es compared with the best-fit models. The black solid line (and also the blue for SN2013hx) refers to the magnetar model, while the red dot-dashed (and also the green for SN2013hx) refers to the CSM+Ni model. Symbols are the same of Fig. 15.

tra and temperature evolution to those of fast-declining type II SNe but delayed by 15-20 days in a similar fashion to what shown between SLSNe I and stripped envelope SNe. In addition they share similar faint host galaxies as those of SLSNe I.

For these reasons we fit our light curves with our semianalytical diffusion model presented in Inserra et al. (2013b) in which a rapidly spinning magnetar deposits its rotational energy into a supernova explosion, through magnetic dipole radiation, and significantly enhances the luminosity ${ }^{6}$. Then, we treat for $\gamma$-ray leakage from ejecta as done in Chen et al. (2015), which is similar to the prescription of Wang et al. (2015). The magnetar luminosity depends primarily on two parameters, the magnetic field strength $\mathrm{B}_{14}$ (expressed in terms of $10^{14} \mathrm{G}$ ) and the initial spin period $\mathrm{P}_{\mathrm{ms}}$ (in milliseconds). As previously done with the interaction model, we used an opacity $\kappa=0.34 \mathrm{~cm}^{2} \mathrm{~g}^{-1}$. Table 6 lists the bestfit parameters for each object, and Fig. 14 shows the fits (black solid line). As the reduced $\chi^{2}\left(\chi_{\text {red }}^{2}=\chi^{2} /\right.$ d.o.f. $)$ fitting gives good matches to models without ${ }^{56} \mathrm{Ni}$, we have no need to introduce ${ }^{56} \mathrm{Ni}$ as an additional free parameter. As in the case of CSM model fitting for SN2013hx, we do two fits (only using data up to 40 days and then using all data up to 178 days), which in this case are very similar. On

6 The semi-analytical code is available at https://star.pst. qub.ac.uk/wiki/doku.php/users/ajerkstrand/start 
Table 5. Best-fit parameters for the CSM+Ni model, which has nine free parameters.

\begin{tabular}{lccccc}
\hline \hline Object & $\begin{array}{c}\mathrm{t}_{\text {rise }} \\
(\text { days })\end{array}$ & $\begin{array}{c}\mathrm{M}_{\mathrm{CSM}} \\
\left(\mathrm{M}_{\odot}\right)\end{array}$ & $\begin{array}{c}\mathrm{M}\left({ }^{56} \mathrm{Ni}\right) \\
\left(\mathrm{M}_{\odot}\right)\end{array}$ & $\begin{array}{c}\mathrm{M}_{\mathrm{ej}} \\
\left(\mathrm{M}_{\odot}\right)\end{array}$ & $\chi^{2} /$ d.o.f. \\
\hline SN2013hx* & $40.35 / 40.35$ & $2.35 / 2.37$ & $2.02 / 0.61$ & $9.81 / 10.48$ & $0.8 / 20$ \\
PS15br & 31.90 & 2.09 & 0.00 & 12.94 & $500.5 / 55$ \\
SN2008es & 36.41 & 2.75 & 1.14 & 9.46 & $5.4 / 30$ \\
\hline
\end{tabular}

* The first values refer to the model considering only the first 40 days, while the second refer to the model considering up to 178 days.

Table 6. Best-fit parameters for magnetar modelling, which has four free parameters, of the bolometric light curves and $\chi^{2} /$ d.o.f. value, together with the derived parameters (last two columns).

\begin{tabular}{|c|c|c|c|c|c|c|c|}
\hline Object & $\begin{array}{c}\tau_{\mathrm{m}} \\
\text { (day) }\end{array}$ & $B_{14}$ & $P_{\mathrm{ms}}$ & $\begin{array}{c}t_{0} \\
\text { (MJD) }\end{array}$ & $\chi^{2} /$ d.o.f. & $\begin{array}{c}E^{\mathrm{mag}} \\
\left(10^{51} \mathrm{erg}\right)\end{array}$ & $\begin{array}{c}M_{\mathrm{ej}} \\
\left(\mathrm{M}_{\odot}\right)\end{array}$ \\
\hline SN2013hx * & $36.71 / 38.15$ & $1.31 / 0.89$ & $2.22 / 2.29$ & $56643.20 / 56641.57$ & $0.6 / 151.61 / 23$ & $3.38 / 5.24$ & $6.95 / 5.20$ \\
\hline PS15br & 17.60 & 1.61 & 6.38 & 57056.27 & $161.5 / 50$ & 0.39 & 1.93 \\
\hline SN2008es & 36.58 & 0.97 & 2.35 & 54578.28 & $5.0 / 25$ & 2.66 & 5.71 \\
\hline
\end{tabular}

* The first values refer to the model considering only the first 40 days, while the second refer to the model considering up to 178 days.

the other hand, for PS15br we only fit the early light curve up to 102 days. The light curves of our two objects are quite well reproduced but the late luminosity of the model for SN2013hx is better reproduced by the 40 days magnetar model. These data points may already have an additional luminosity contribution due to some late interaction (as seen in the late spectra), and hence a light curve dimmer than these points would favour such a model. This is also true for PS15br where the magnetar model is dimmer than the late time points where the luminosity is driven by interaction. The CSM models are too bright to describe SN2013hx data after $\sim 180$ days. Furthermore if we remove the ${ }^{56} \mathrm{Ni}$ contribution, we get a worse $\chi_{\text {red }}^{2}$ and a luminosity at late time still brighter than the data. The magnetar fit for PS15br is better than that of the CSM as testified by the $\chi_{\text {red }}^{2}$ values of Tables $5 \& 6$, despite the latter having a higher number of free parameters. The magnetar fit to SN2008es is not as satisfactory as the CSM one, as the data show a faster rise than the best fit model. However, the results of SN2008es fit are in agreement with that of Kasen \& Bildsten $(2010)^{7}$. One may ask why SN2013hx and SN2008es end up with different results if their light curve behaviour is so similar. As shown in the right panel of Fig. 14, the reason is that we have different time coverage for each. The light curve data for SN2008es stretch to 80 days after peak where as the data for SN2013hx stops at 50 days, and there are more pre-peak detection points for SN2013hx than for SN2008es (which has only limits before -10 days).

We further estimated velocity and temperature evolution from the light curve fits to ejecta mass and kinetic energy. In Fig. 15 we show that the temperature evolution of the magnetar model matches the observed ones reasonably well and better than the interaction model, which produces results which are always too hot. Photospheric velocities are slightly faster than the Fe II velocities, which trace

7 This is expected, since in Inserra et al. (2013b) we already tested our code with respect to that of Kasen \& Bildsten (2010) the photospheric evolution. The sudden drop to zero of both temperature and velocity evolution of the best fit model for PS15br happens because more than half of the core has been exposed. This is a consequence of the low energy and ejecta mass needed to fit the light curve.

Another possible central engine is that of the fall-back accretion (Dexter \& Kasen 2013), which can give a similar asymptotic behaviour of the light curve $\left(\mathrm{L}_{\mathrm{t}} \propto t^{-5 / 3}\right)$ with respect to the magnetar engine but would have lower luminosity than that observed after 180 days in SN2013hx. However, this scenario implies photospheric velocities higher than that shown by our sample and would fail to reproduce PS15br light curve since models considering $\mathrm{H}$ recombination have longer rise times and a bump 200 or 300 days after the explosion. To better investigate this scenario a dedicated fit for these objects is required.

\section{CONCLUSION}

We have presented extensive photometric and spectroscopic coverage of two superluminous, hydrogen-rich supernovae with light curves covering from weeks before peak up to 260 (SN2013hx) and 365 (PS15br) days. We then analysed the two objects together with the only other SLSN II (SN2008es) to search for similar observational behaviours suggesting their existence as superluminous events different than superluminous interacting event (i.e. SLSNe IIn) or SLSNe I.

SN2013hx has $\mathrm{M}_{g}=-21.7 \mathrm{mag}$ and $\mathrm{L}_{\text {bolometric }} \approx 2.75 \times$ $10^{44} \mathrm{erg} \mathrm{s}^{-1}$ at peak, while PS15br shows $\mathrm{M}_{g} \sim-20.2$ and $\mathrm{M}_{U} \leq-20.8 \mathrm{mag}$ and $\mathrm{L}_{\text {bolometric }} \approx 4.15 \times 10^{43} \mathrm{erg} \mathrm{s}^{-1}$ at maximum light. The former has a post peak light curve decline similar to that of SN2008es and normal type IIL, whereas PS15br shows an initial decline comparable to that of SN2013hx and SN2008es/ and a second, slower decline similar to those shown by transitional type IIn/II events at normal luminosity. Although PS15br luminosity is fainter than SN2013hx and SN2008es, we have consider it a "bona fide" SLSN II due to its spectroscopic evolution similar to 
that of SN2013hx and PS15br and a spectrophotometric evolution dissimilar to any other H-rich SN in a similar luminosity space.

For PS15br we also obtained an epoch of broadband polarimetry at 6 days after maximum. After correcting for the ISP and the polarisation bias, we retrieved a polarised value of $P=0.94 \pm 0.17 \%$. However, this value strongly depends on the assumed ISP and hence no conclusive evidence on the source of the polarisation associated with PS15br can be derived.

SLSNe II (namely SN2008es, SN2013hx, PS15br) show a spectroscopic evolution dominated by Balmer, He, Ca, $\mathrm{Fe}$ and other metal lines and an emission feature at early times at $\sim 4600 \AA$ identified as C III/N III. SN2013hx and PS15br show a late interaction (phase $>140 \mathrm{~d}$ and $>250 \mathrm{~d}$, respectively) due to an asymmetric, clumpy CSM that dominates the late light curve behaviour. Regardless of the peak luminosity, SLSNe II spectroscopic and temperature evolution resembles those of fast-declining type II (or type IIL) $\mathrm{SNe}$ with a delay of 15-20 days. This suggest as the transients are linked to type II SNe in a similar fashion as SLSNe I are linked to type Ic SNe.

We applied two semi-analytical codes to fit the light curves of all SLSNe II. The first based on the interaction between the ejecta and a dense uniform shell of H-rich material, while the second based on a diffusion model with energy input from a spinning down magnetar. We were able to reproduce the available bolometric light curve with $2.1 \lesssim \mathrm{M}_{\mathrm{CSM}}\left(\mathrm{M}_{\odot}\right) \lesssim 2.8$ and $9.5 \lesssim \mathrm{M}_{\mathrm{ej}}\left(\mathrm{M}_{\odot}\right) \lesssim 13.0$. While the late time light curve exhibited by SN2013hx and PS15br and driven by interaction can be fitted with a wind of 2.6 and 2.3 $\mathrm{M}_{\odot}$ or a dense shell of 4.7 and $3.9 \mathrm{M}_{\odot}$, respectively All light curves are also reproduced with feasible physical values for a magnetar including the data at 140-180 days of SN2013hx, when the luminosity of our best fit model is lower than the data, which are already due to the aforementioned late interaction. To fit them we require $0.9 \lesssim B_{14} \lesssim 1.6,2.2 \lesssim P_{\mathrm{ms}} \lesssim 6.4$ consistent with $B$ of known galactic magnetars $\left(B_{14} \sim 1-10\right)$ and with physically plausible periods $\left(P_{\mathrm{ms}}>1\right)$. We derived energies of $0.4 \lesssim E^{\mathrm{mag}}\left(10^{51} \mathrm{erg}\right) \lesssim 5.2$ and ejected masses of $1.9 \lesssim M_{\mathrm{ej}}\left(\mathrm{M}_{\odot}\right) \lesssim 6.9$. Both scenarios have their weaknesses. The high ejecta masses retrieved by the interaction model and the spectroscopic similarities to bright, linear type II regardless of the peak luminosity, would tend to disfavour of the interaction scenario. On the other hand, these characteristics can be better explained by the magnetar scenario. However, at least for the case of PS15br, some weak interaction is present for the majority of the photospheric evolution in a similar fashion to hat found for same SLSN I (Inserra et al. 2017c). In addition, the interaction shown at late phase by SN2013hx and PS15br does not allow a firm conclusion and suggests as interaction could play a role in the evolution of SLSNe II.

Despite our dataset and analysis of this first sample of SLSNe II, open questions remain

- Do SLSNe II with interaction-free light curves exist? What should be their luminosity and what are the line profiles expected in a truly nebular spectrum?

- What is the role of metallicity in the progenitor stars evolution that will produce a H-rich SLSNe? It appears that they are all associated with faint dwarf galaxies and possibly low metallicity progenitors as those of SLSNe I.

- Can the geometry of the explosion play a role in the observables and/or shed light on the powering mechanism or their light curves?

As for the case of SLSNe I, to address these topics, high quality data and their modelling at early phase or in an interaction-free nebular phase are needed to determine the ejecta masses, composition and the mass of ${ }^{56} \mathrm{Co}$ contributing to the luminosity. This seems the most likely way to make progress.

\section{ACKNOWLEDGMENTS}

SJS acknowledges funding from the European Research Council under the European Union's Seventh Framework Programme (FP7/2007-2013)/ERC Grant agreement $\mathrm{n}^{\mathrm{o}}$ [291222] and STFC grants ST/I001123/1 and $\mathrm{ST} / \mathrm{L} 000709 / 1$. MF is supported by a Royal Society - Science Foundation Ireland University Research Fellowship EEEG acknowledges support for this work by the Deutsche Forschungsgemeinschaft through the TransRegio project TRR33 'The Dark Universe'. SB is partially supported by the PRIN-INAF 2014 project Transient Universe: unveiling new types of stellar explosions with PESSTO. Support for I.A. was provided by NASA through the Einstein Fellowship Program, grant PF6-170148. KM acknowledges support from the STFC through an Ernest Rutherford Fellowship. SP and RC acknowledge support from EU/FP7ERC grant no [615929]. SS acknowledges support from CONICYT-Chile FONDECYT 3140534, Basal-CATA PFB06/2007, and Project IC120009 "Millennium Institute of Astrophysics (MAS)" of Iniciativa Científica Milenio del Ministerio de Economía, Fomento y Turismo. This work was partly supported by the European Union FP7 programme through ERC grant number 320360. Part of this research was conducted by the Australian Research Council Centre of Excellence for All-sky Astrophysics (CAASTRO), through project number CE110001020 Pan-STARRS is supported by the University of Hawaii and the National Aeronautics and Space Administration's Planetary Defense Office under Grant No. NNX14AM74G. The Pan-STARRS1 Sky Surveys have been made possible through contributions by the Institute for Astronomy, the University of Hawaii, the PanSTARRS Project Office, the Max Planck Society and its participating institutes, the Max Planck Institute for Astronomy, Heidelberg and the Max Planck Institute for Extraterrestrial Physics, Garching, The Johns Hopkins University, Durham University, the University of Edinburgh, the Queen's University Belfast, the Harvard-Smithsonian Center for Astrophysics, the Las Cumbres Observatory Global Telescope Network Incorporated, the National Central University of Taiwan, the Space Telescope Science Institute, and the National Aeronautics and Space Administration under Grant No. NNX08AR22G issued through the Planetary Science Division of the NASA Science Mission Directorate, the National Science Foundation Grant No. AST1238877, the University of Maryland, Eotvos Lorand University (ELTE), and the Los Alamos National Laboratory. This work is based (in part) on observations collected at the European Organisation for Astronomical Research in the 
Southern Hemisphere, Chile as part of PESSTO, (the Public ESO Spectroscopic Survey for Transient Objects Survey) ESO programs188.D-3003, 191.D-0935. Some of the data presented herein were obtained at the Palomar Observatory, California Institute of Technology. The Liverpool Telescope is operated on the island of La Palma by Liverpool John Moores University in the Spanish Observatorio del Roque de los Muchachos of the Instituto de Astrofisica de Canarias with financial support from the UK Science and Technology Facilities Council.

\section{REFERENCES}

Agnoletto I., et al., 2009, ApJ, 691, 1348

Alam S., et al., 2015, ApJS, 219, 12

Alard C., 2000, A\&AS, 144, 363

Ambikasaran S., Foreman-Mackey D., Greengard L., Hogg D. W., O'Neil M., 2014, preprint, (arXiv: 1403.6015)

Anderson J. P., et al., 2014a, MNRAS, 441, 671

Anderson J. P., et al., 2014b, ApJ, 786, 67

Arcavi I., et al., 2016, ApJ, 819, 35

Baltay C., et al., 2007, PASP, 119, 1278

Barbon R., Ciatti F., Rosino L., 1982, A\&A, 116, 35

Barkat Z., Rakavy G., Sack N., 1967, Physical Review Letters, 18,379

Benetti S., et al., 2014, MNRAS, 441, 289

Benetti S., et al., 2016, MNRAS, 456, 3296

Bessell M., Bloxham G., Schmidt B., Keller S., Tisserand P., Francis P., 2011, PASP, 123, 789

Bishop C., 2006, Pattern Recognition and Machine Learning. 1613-9011, Springer-Verlag New York

Blanton M. R., Roweis S., 2007, AJ, 133, 734

Blinnikov S. I., Bartunov O. S., 1993, A\&A, 273, 106

Bose S., et al., 2015, ApJ, 806, 160

Botticella M. T., et al., 2010, ApJ, 717, L52

Brown T. M., et al., 2013, PASP, 125, 1031

Campbell H., et al., 2014, The Astronomer's Telegram, 5912

Cappellaro E., Danziger I. J., Turatto M., 1995, MNRAS, 277, 106

Chatzopoulos E., Wheeler J. C., Vinko J., 2012, ApJ, 746, 121

Chatzopoulos E., Wheeler J. C., Vinko J., Horvath Z. L., Nagy A., 2013, ApJ, 773, 76

Chen T.-W., et al., 2015, MNRAS, 452, 1567

Chevalier R. A., Irwin C. M., 2011, ApJ, 729, L6

Childress M. J., Vogt F. P. A., Nielsen J., Sharp R. G., 2014, Ap\&SS, 349, 617

Chugai N. N., 1985, Soviet Astronomy Letters, 11, 148

Chugai N. N., Chevalier R. A., Utrobin V. P., 2007, ApJ, 662, 1136

Dessart L., Hillier D. J., 2005, A\&A, 437, 667

Dexter J., Kasen D., 2013, ApJ, 772, 30

Dopita M., et al., 2010, Ap\&SS, 327, 245

Drake A. J., et al., 2009, ApJ, 696, 870

Elmhamdi A., et al., 2003, MNRAS, 338, 939

Faran T., et al., 2014, MNRAS, 445, 554

Fassia A., et al., 2001, MNRAS, 325, 907

Fesen R. A., et al., 1999, AJ, 117, 725

Fraley G. S., 1968, Ap\&SS, 2, 96

Fraser M., et al., 2015, The Astronomer's Telegram, 7209

Freudling W., Romaniello M., Bramich D. M., Ballester P., Forchi V., García-Dabló C. E., Moehler S., Neeser M. J., 2013, A\&A, 559, A96

Gal-Yam A., 2012, Science, 337, 927

Gal-Yam A., et al., 2007, ApJ, 656, 372

Gal-Yam A., et al., 2014, Nature, 509, 471

Galbany L., et al., 2016, AJ, 151, 33
Gall E. E. E., et al., 2015, A\&A, 582, A3

Gezari S., et al., 2009, ApJ, 690, 1313

Gutiérrez C. P., et al., 2017a, preprint, (arXiv:1709.02487)

Gutiérrez C. P., et al., 2017b, preprint, (arXiv:1709.02799)

Hoflich P., 1991, A\&A, 246, 481

Hogg D. W., Baldry I. K., Blanton M. R., Eisenstein D. J., 2002, ArXiv Astrophysics e-prints,

Huber M., et al., 2015, The Astronomer's Telegram, 7153

Inserra C., Smartt S. J., 2014, ApJ, 796, 87

Inserra C., et al., 2011, MNRAS, 417, 261

Inserra C., et al., 2012a, MNRAS, 422, 1122

Inserra C., Baron E., Turatto M., 2012b, MNRAS, 422, 1178

Inserra C., et al., 2013a, A\&A, 555, A142

Inserra C., et al., 2013b, ApJ, 770, 128

Inserra C., et al., 2015, ApJ, 799, L2

Inserra C., et al., 2016a, MNRAS, 459, 2721

Inserra C., Bulla M., Sim S. A., Smartt S. J., 2016b, ApJ, 831, 79

Inserra C., Prajs S., Gutierrez C. P., Smith C. A. M., Sullivan M., 2017a, preprint, (arXiv:1711.03787)

Inserra C., et al., 2017b, preprint, (arXiv:1710.09585)

Inserra C., et al., 2017c, MNRAS, 468, 4642

Ivezic Z., Connolly A. J., VanderPlas J. T., Gray A., 2014, Statistics, Data Mining, and Machine Learning in Astronomy: A Practical Python Guide for the Analysis of Survey Data. Princeton University Press, Princeton, NJ, USA

Janka H.-T., 2012, Annual Review of Nuclear and Particle Science, 62,407

Kangas T., et al., 2016, MNRAS, 456, 323

Kasen D., Bildsten L., 2010, ApJ, 717, 245

Keller S., Bessell M., Schmidt B., Francis P., 2007, in Sterken C., ed., Astronomical Society of the Pacific Conference Series Vol. 364, The Future of Photometric, Spectrophotometric and Polarimetric Standardization. p. 177

Kim A., Goobar A., Perlmutter S., 1996, PASP, 108, 190

Kim A. G., et al., 2013, ApJ, 766, 84

Kozma C., Fransson C., 1998, ApJ, 497, 431

Krühler T., et al., 2015, A\&A, 581, A125

Lantz B., et al., 2004, in Mazuray L., Rogers P. J., Wartmann R., eds, Proc. SPIEVol. 5249, Optical Design and Engineering. pp 146-155, doi:10.1117/12.512493

Leloudas G., et al., 2015a, MNRAS, 449, 917

Leloudas G., et al., 2015b, ApJ, 815, L10

Leloudas G., et al., 2017, ApJ, 837, L14

Li W., et al., 2011, MNRAS, 412, 1441

Lunnan R., et al., 2014, ApJ, 787, 138

Lunnan R., et al., 2016, ApJ, 831, 144

Magnier E. A., et al., 2013, ApJS, 205, 20

Maguire K., et al., 2010, MNRAS, 404, 981

Mazzali P. A., Sullivan M., Pian E., Greiner J., Kann D. A., 2016, MNRAS, 458, 3455

Miller A. A., et al., 2009, ApJ, 690, 1303

Moriya T. J., Yoon S.-C., Gräfener G., Blinnikov S. I., 2017, MNRAS, 469, L108

Morozova V., Piro A. L., Valenti S., 2017, ApJ, 838, 28

Nicholl M., et al., 2014, MNRAS, 444, 2096

Nicholl M., et al., 2015, MNRAS, 452, 3869

Ofek E. O., et al., 2007, ApJ, 659, L13

Papadopoulos A., et al., 2015, MNRAS, 449, 1215

Pastorello A., et al., 2010, ApJ, 724, L16

Pastorello A., et al., 2013, ApJ, 767, 1

Pastorello A., et al., 2015, MNRAS, 449, 1954

Patat F., Romaniello M., 2006, PASP, 118, 146

Patat F., et al., 2001, ApJ, 555, 900

Pignata G., et al., 2004, MNRAS, 355, 178

Polshaw J., et al., 2016, A\&A, 588, A1

Poole T. S., et al., 2008, MNRAS, 383, 627 
Pozzo M., Meikle W. P. S., Fassia A., Geballe T., Lundqvist P., Chugai N. N., Sollerman J., 2004, MNRAS, 352, 457

Prajs S., et al., 2017, MNRAS, 464, 3568

Pritchard T. A., et al., 2012, ApJ, 750, 128

Quimby R. M., Aldering G., Wheeler J. C., Höflich P., Akerlof C. W., Rykoff E. S., 2007, ApJ, 668, L99

Quimby R. M., et al., 2011, Nature, 474, 487

Quimby R. M., Yuan F., Akerlof C., Wheeler J. C., 2013, MNRAS, 431, 912

Rasmussen C. E., Williams C. K. I., 2006, Gaussian Processes for Machine Learning. The MIT Press, Cambridge, MA

Sanders N. E., et al., 2015, ApJ, 799, 208

Scalzo R., Yuan F., Childress M., Tucker B., Schmidt B., 2013, The Astronomer's Telegram, 5480

Scalzo R., et al., 2014a, MNRAS, 440, 1498

Scalzo R., et al., 2014b, Central Bureau Electronic Telegrams, 3836

Schlafly E. F., Finkbeiner D. P., 2011, ApJ, 737, 103

Schlafly E. F., et al., 2012, ApJ, 756, 158

Schulze S., et al., 2016, preprint, (arXiv:1612.05978)

Smartt S. J., 2009, ARA\&A, 47, 63

Smartt S. J., 2015, Publ. Astron. Soc. Australia, 32, e016

Smartt S. J., et al., 2015, A\&A, 579, A40

Smartt S. J., et al., 2016, MNRAS, 462, 4094

Smith N., 2014, ARA\&A, 52, 487

Smith N., McCray R., 2007, ApJ, 671, L17

Smith N., Owocki S. P., 2006, ApJ, 645, L45

Smith N., Humphreys R. M., Davidson K., Gehrz R. D., Schuster M. T., Krautter J., 2001, AJ, 121, 1111

Smith N., et al., 2007, ApJ, 666, 1116

Smith N., et al., 2015, MNRAS, 449, 1876

Steele I. A., et al., 2004, in Oschmann Jr. J. M., ed., Proc. SPIEVol. 5489, Ground-based Telescopes. pp 679-692, doi: $10.1117 / 12.551456$

Stritzinger M., et al., 2002, AJ, 124, 2100

Tomasella L., et al., 2013, MNRAS, 434, 1636

Tonry J. L., et al., 2012, ApJ, 750, 99

Turatto M., et al., 2000, ApJ, 534, L57

Udalski A., Szymański M. K., Szymański G., 2015, Acta Astron., 65,1

Umeda H., Nomoto K., 2008, ApJ, 673, 1014

Uomoto A., Kirshner R. P., 1986, ApJ, 308, 685

Valenti S., et al., 2014, MNRAS, 438, L101

Valenti S., et al., 2015, MNRAS, 448, 2608

Valenti S., et al., 2016, MNRAS, 459, 3939

Wang L., Wheeler J. C., 2008, ARA\&A, 46, 433

Wang L., Howell D. A., Höflich P., Wheeler J. C., 2001, ApJ, 550, 1030

Wang S. Q., Wang L. J., Dai Z. G., Wu X. F., 2015, ApJ, 799, 107

Woosley S. E., Weaver T. A., 1995, ApJS, 101, 181

Woosley S. E., Blinnikov S., Heger A., 2007, Nature, 450, 390

Xu Y., McCray R., Oliva E., Randich S., 1992, ApJ, 386, 181

Yan L., et al., 2015, ApJ, 814, 108

Yan L., et al., 2017, ApJ, 840, 57

Yaron O., Gal-Yam A., 2012, PASP, 124, 668

Yaron O., et al., 2017, Nature Physics, 13, 510

de Jaeger T., et al., 2017, preprint, (arXiv:1709.01513) 


\section{APPENDIX A: OBSERVATIONS AND DATA REDUCTION}

Both objects were immediately selected by PESSTO as follow-up science targets and a combination of optical, near infrared (NIR) and ultraviolet (UV) photometric monitoring was carried out. The epochs of all the data, as well as the telescopes and instruments used are listed in Appendix C.

All images were reduced (trimmed, bias subtracted and flat-fielded) by the SMT, PSST, Las Cumbres Observatory (LCO, Brown et al. 2013), Liverpool Telescope (LT, Steele et al. 2004), CRTS and PESSTO pipelines. Photometric zero-points and colour terms were computed using observations of standard fields $(U, B, V, R, I$ in Vega and $g, r, i, z$ in $\mathrm{AB}$ system). We then calibrated the magnitudes of local stellar sequences shown in Appendix D. The average magnitudes of the local-sequence stars were used to calibrate the photometric zero-points in non-photometric nights. The near-infrared $J, H, K$ photometry was carried out on SOFI on the NTT and the data were again reduced as discussed in Smartt et al. (2015). Magnitudes were calibrated to the Two Micron All Sky Survey system using local sequence stars. The magnitudes of the SNe, obtained through a point spread function (PSF) fitting technique using standard procedures in $\mathrm{IRAF}^{8}$, were measured on the final images. The uncertainties reported in the Appendix C Tables C1, C2 \& C3 were estimated by combining in quadrature the errors of photometric calibration and those on the instrumental magnitudes. When the objects were not detected, limiting magnitudes were estimated by placing artificial stars of different magnitudes at the expected SN positions. When necessary - i.e. after 170d for SN2013hx and 100d for PS15br - we applied a template subtraction technique (through the HOTPANTS $^{9}$ package based on the algorithm presented in Alard 2000). The instruments used to obtain the templates were NTT for SN2013hx and PSST for PS15br. The same frames were used to measure the host magnitudes and host NIR limit magnitudes (see Sections $2.1 \& 2.2$ ).

Differences between passbands were taken into account applying a passband correction (P-correction) using the SuperNova Algorithm for P-correction (SNAP) in the S3 package (see Appendix B for further details). This P-correction is similar to the S-correction (Stritzinger et al. 2002; Pignata et al. 2004).

Ultraviolet ( $u v w 2, u v m 2, u v w 1$; see Poole et al. 2008) observations, obtained by UVOT on board of the Swift satellite (P.I. Margutti for SN2013hx and Inserra for PS15br) were reduced using the HEASARC ${ }^{10}$ software. We analysed these publicly available data independently. Swift $u, b, v$ aperture magnitudes were transformed to Johnson system by applying a shift after comparison with optical ground-based data taken close in time (see Inserra et al. 2011; Pastorello et al. 2013, for further details in the procedure).

Host galaxies photometry was carried out through aper-

8 Image Reduction and Analysis Facility, distributed by the National Optical Astronomy Observatories, which are operated by the Association of Universities for Research in Astronomy, Inc, under contract to the National Science Foundation.

9 http://www.astro.washington.edu/users/becker/hotpants.html 10 NASA's High Energy Astrophysics Science Archive Research Center ture photometry within IRAF/DAOPHOT package, and we used the same aperture size to measure the flux of local secondary standards in the field. The griz photometry was calibrated against Pan-STARRS1 sequence stars, or local secondary stars, and converted to the SDSS photometric system; while JHK was calibrated against the 2MASS catalogue.

The journal of spectroscopic observations is listed in Table C7. The majority of the follow-up spectra where taken with PESSTO and the NTT. Integral field spectra were also taken with the ANU $2.3 \mathrm{~m}$ telescope + the Wide Field Spectrograph (WiFeS, Dopita et al. 2010) at Siding Spring Observatory in New South Wales, Australia. A final spectrum of PS15br was taken with the SuperNova Integral Field Spectrograph (SNIFS, Lantz et al. 2004) at the $2.2 \mathrm{~m}$ University Hawaii Telescope. A series of spectra of SN2013hx was taken with the VLT+FORS2 ${ }^{11}$ in the second season of observations, and combined together into one deep late-time spectrum. These FORS2 exposures had a total of $11 \times 1230 \mathrm{sec}$ and $1 \times 1800$ and were taken on the nights of 22-24 October 2014 through service mode on the VLT (giving an on-sky total exposure time of $4 \mathrm{hr} 15 \mathrm{~min} 30 \mathrm{sec}$ ). On the other hand, two spectra of PS15br were taken with the VLT+XShooter in service mode. We used the custom made pipeline described in Krühler et al. (2015) which takes the ESO pipeline produced 2D spectral products (from REFLEX; Freudling et al. 2013) and uses optimal extraction with a Moffat profile fit. This pipeline produced flux calibrated spectra with rebinned dispersions of $0.4 \AA_{\text {pix }}^{-1}$ in the UVB+VIS arms and $0.6 \AA_{\mathrm{pix}}^{-1}$ in the NIR arm. The NTT spectral data were reduced using the PESSTO pipeline (Smartt et al. 2015), while the VLT+FORS2 spectra were reduced in the REFLEX environment (Freudling et al. 2013) and the SNIFS spectrum using standard IRAF procedures as described in Smartt et al. (2016). Optimal extraction of the spectra was adopted to improve the final signal-to-noise $(\mathrm{S} / \mathrm{N})$ ratio. Wavelength calibration was performed using spectra of comparison lamps acquired with the same configurations as the SN observations. Atmospheric extinction correction was based on tabulated extinction coefficients for each telescope site. The WiFeS spectra were reduced with PYWIFES $^{12}$ package (Childress et al. 2014) to produce data cubes, from which the final spectra were obtained using a PSF weighted extraction routine. Flux calibration was performed using spectro-photometric standard stars observed on the same nights with the same set-up as the SLSNe. The flux calibration was checked by comparison with the photometry, integrating the spectral flux transmitted by standard griz filters and adjusted by a multiplicative factor when necessary. The resulting flux calibration is accurate to within $0.1-0.2 \mathrm{mag}$.

All reduced spectra (and calibrated NIR images), taken at the NTT before 2014 April 30, are available from the ESO Science Archive Facility as PESSTO SSDR2 and details of data access are provided on the PESSTO website ${ }^{13}$. Data taken after this date are part of the SSDR3. All spectra will

11 Very Large Telescope + FOcal Reducer and low dispersion Spectrograph

12 http://www.mso.anu.edu.au/pywifes/

13 www.pessto.org 
also be available through WISeREP ${ }^{14}$ (Yaron \& Gal-Yam 2012).

\section{APPENDIX B: S3 PACKAGE}

The S3 package is a python suite publicly available at https: //github.com/cinserra and accepts fits, txt, dat and ascii files as input. It contains the following programs.

\section{B1 snake}

The SuperNova Algorithm for $K$-correction Evaluation (SNAKE) and its light version developed to handle multiple input files in a less interactive fashion (SNAKELOOP) allow to evaluate the $K$-correction from the observed passband (P) to the rest-frame, desired passband (R) according to the formula defined in Hogg et al. (2002); Blanton \& Roweis (2007):

$$
\begin{array}{r}
K_{\mathrm{PR}}=-2.5 \log \left[\frac{1}{1+z}\right] \\
-2.5 \log \left[\frac{\int d \lambda_{\mathrm{o}} \lambda_{\mathrm{o}} L_{\lambda}\left(\lambda_{\mathrm{o}} /(1+z)\right) P\left(\lambda_{\mathrm{o}}\right) \int d \lambda_{\mathrm{e}} \lambda_{\mathrm{e}} g_{\lambda}^{R}\left(\lambda_{\mathrm{e}}\right) R\left(\lambda_{\mathrm{e}}\right)}{\int d \lambda_{\mathrm{o}} \lambda_{\mathrm{o}} g_{\lambda}^{P}\left(\lambda_{\mathrm{o}}\right) P\left(\lambda_{\mathrm{o}}\right) \int d \lambda_{\mathrm{e}} \lambda_{\mathrm{e}} L_{\lambda}\left(\lambda_{\mathrm{e}}\right) R\left(\lambda_{\mathrm{e}}\right)}\right],
\end{array}
$$

where $\mathrm{P}(\lambda)$ and $\mathrm{R}(\lambda)$ are the filter response per unit photon, $\mathrm{g}_{\lambda}^{\text {passband }}$ are the flux densities per unit wavelength for the standard source for $\mathrm{P}$ and $\mathrm{R}, \lambda_{\mathrm{o}}$ refers to the observer frame and $\lambda_{\mathrm{e}}$ to the rest-frame. We note that the S3 package uses flux and not density flux, hence the zero points are also treated accordingly. GALEX FUV and NUV; UVOT $u v w 2$, uvm2, uvw1; Johnson $U B V R I$; Sloan $u, g, r, i, z$; 2MASS $J, H, K$; Euclid NIR $Y, J, H$ (Inserra et al. 2017b) and 4000 $\AA$ and $5200 \AA$ passbands (created for the use of SLSNe as cosmological probes in Inserra \& Smartt 2014) are recognised by the program and the cross $K$-correction - which is when the observed and rest-frame filter shapes are the most similar - is suggested. In the case that more than $50 \AA$ of one of the two filters chosen are not covered by the observer frame or rest-frame spectrum, the program allows the user to combine the spectrum with the best black-body fit to the data. The output of the program is the $K$-correction $\left(K_{\mathrm{PR}}\right)$ and it is related to the apparent and absolute magnitude as follows:

$\mathrm{M}_{\mathrm{R}}=\mathrm{m}_{\mathrm{P}}-\left(5 \log \mathrm{D}_{\mathrm{L}}+25\right)-\mathrm{A}_{\mathrm{P}}-K_{\mathrm{PR}}$,

in which $M_{R}$ is the absolute magnitude in the rest-frame filter, $m_{P}$ is the apparent magnitude in the observed filter, $D_{L}$ is the distance luminosity of the source, $A_{P}$ is the foreground reddening extinction toward the source in the observed band. Assuming as a first approximation that the terms are uncorrelated and evaluating the single term errors as their variance, the overall error on the $K$-correction is a root mean square:

$\sigma_{K}=\sqrt{\frac{\sigma_{z}^{2}+\sigma_{\mathrm{ZP}_{\lambda_{\mathrm{o}}}}^{2}+\sigma_{\mathrm{ZP}_{\lambda_{\mathrm{e}}}}^{2}+\sigma_{\mathrm{BB}}^{2}}{\mathrm{~N}}}$,

14 http://wiserep.weizmann.ac.il/home where $\sigma_{z}$ is the error on the redshift, which is set by default to be \pm 0.005 and can be changed by the user; $\sigma_{Z P_{\lambda}}$ are the errors on the zero points for the observed (o) and rest-frame (e) filters evaluated from the average of all the differences between the zero points of the standard passbands (in the Vega and AB system) and those of the filters used by the telescopes listed in SNAP (cfr. Section B2); $\sigma_{\mathrm{BB}}$ is the error on the assumption that a blackbody can be used to measure the wavelength uncovered by the spectrum for the filter chosen; $\mathrm{N}$ is the number of errors considered. Other two errors - related to the spectra template used in the case a spectrum of the object for the same, or very close in time, epoch of the photometry are not available - should be considered, but the program does not handle that since they are highly dependent to the nature and the evolutionary phase of the object for which the $K$-correction is needed.

In order to test the program reliability we compared our results with those published by Kim et al. (1996) finding similar values. A previous, beta version, based on IRAF and STDAS was used to calculate $K$-corrections in Inserra \& Smartt (2014); Inserra et al. (2015), while this version has been used by Gall et al. (2015) and Polshaw et al. (2016). Furthermore, the program can also evaluate the $K$-correction when reddening is also applied and hence directly evaluate the term $-\left(\mathrm{A}_{\mathrm{P}}+K_{\mathrm{PR}}\right)$ of equation $\mathrm{B} 2$. However, this is not the correct way and the additional errors related to the assumed reddening and reddening law are not accounted for.

\section{B2 SNAP}

The SuperNova Algorithm for P-correction (SNAP) evaluate a P-correction given by:

$\mathrm{P}_{\lambda}=\mathrm{F}_{\lambda} \times \mathrm{QE}_{\lambda}$

where $\mathrm{F}_{\lambda}$ is the filter transmission function and $\mathrm{QE}_{\lambda}$ is the quantum efficiency of the detector. In contrast with the S-correction, the program does not take in account the lens throughput since, despite the use of a few materials, they are relatively flat across the optical range. However, this might cause some problems at wavelengths bluer than $3400 \AA$, i.e. affecting the $U$ and $u$ passbands. The atmospheric transmission profile is also ignored since SN magnitudes are usually evaluated through sequence stars calibrated with Sloan stars (and hence such correction is already taken in account at this level) or with Landolt stars and subsequent use of programs that apply such correction. We tested a few mirror reflectivity functions and found small dissimilarities between them, quantifiable in magnitudes differences smaller or comparable with the usual photometric errors; hence we decide to not include such additional term. Then the $\mathrm{P}_{\lambda}$ allows to correct the apparent magnitude of a given telescope to that of the standard passband system as follows:

$\mathrm{m}_{\mathrm{P}}=\mathrm{m}_{\mathrm{F}}-\mathrm{P}_{\lambda}$,

where $m_{P}$ is the apparent magnitude with the standard passbands (Johnson $U, B, V, R, I$ or Sloan $u, g, r, i, z$ ) and $\mathrm{m}_{\mathrm{F}}$ is the one measured with photometry at a given telescope. As SNAKE also SNAP evaluate the errors of the P-correction in the same way of equation B3 but without the redshift term and with the rest-frame filter considered as the error on the zero point in flux of the standard passband. 
We retrieved the information needed for the $\mathrm{P}$ correction for the New technology Telescope (NTT; ESO website), La Silla-Quest (LSQ; Baltay et al. 2007, and D. Rabinowitz private communication), SkyMapper (SMT; Bessell et al. 2011, M. Renault and R. Scalzo private communications), the Liverpool Telescope (LT; LT website), the Las Cumbres Observatory Global Network (LCOGT; S. Valenti private communication), the Optical Gravitational Lensing Experiment IV (OGLE-IV; Udalski et al. 2015, and L. Wyrzykowski private communication), the North Optical Telescope (NOT; NOT website), the Pan-STARSS1 survey (PS1; Tonry et al. 2012, and K. W. Smith private communications), the Telescopio Nazionale Galileo (TNG, TNG website), the Copernico Telescope at Cima Ekar in Asiago (EKAR, S. Benetti private communication).

\section{B3 SMS}

The Synthetic Magnitudes from Spectra (SMS) program is just a python version of the STSDAS/HST IRAF tool calcphot. It evaluates the flux in a given passband and converts it in magnitudes (AB or Vega system) for the same filter list used by SNAKE. As SNAKE, it evaluates the errors in the same fashion of SNAKE but without the terms about the redshift and the rest-frame filter

\section{APPENDIX C: TABLES}

\section{APPENDIX D: SEQUENCE STARS AND ADDITIONAL PLOTS}

This paper has been typeset from a $\mathrm{T}_{\mathrm{E}} \mathrm{X} / \mathrm{LAT}_{\mathrm{E}} \mathrm{X}$ file prepared by the author. 
Table C1. $U, B, V, g, r, i, J, H, K$ magnitudes of SN2013hx and assigned errors in brackets.

\begin{tabular}{|c|c|c|c|c|c|c|c|c|c|}
\hline $\begin{array}{c}\text { Date } \\
\mathrm{dd} / \mathrm{mm} / \mathrm{yy}\end{array}$ & MJD & $\begin{array}{c}\text { Phase* } \\
\text { (days) }\end{array}$ & $U$ & $B$ & $V$ & $g$ & $r$ & $i$ & Inst. \\
\hline $01 / 10 / 13$ & 56566.61 & -104.32 & - & - & - & $>20.86$ & $>20.68$ & - & SMT \\
\hline $08 / 10 / 13$ & 56573.58 & -98.16 & - & - & - & - & $>20.71$ & - & SMT \\
\hline $19 / 10 / 13$ & 56584.62 & -88.39 & - & - & - & $>18.97$ & $>19.24$ & - & SMT \\
\hline $28 / 10 / 13$ & 56593.63 & -80.41 & - & - & - & $>19.89$ & $>19.65$ & - & $\mathrm{SMT}$ \\
\hline $01 / 11 / 13$ & 56597.64 & -76.87 & - & - & - & $>20.19$ & $>19.98$ & - & SMT \\
\hline $06 / 11 / 13$ & 56602.60 & -72.48 & - & - & - & $>20.24$ & $>20.02$ & - & $\mathrm{SMT}$ \\
\hline $10 / 11 / 13$ & 56606.64 & -68.90 & - & - & - & $>19.63$ & $>19.64$ & - & SMT \\
\hline $15 / 11 / 13$ & 56611.65 & -64.47 & - & - & - & $>19.20$ & - & - & SMT \\
\hline $20 / 11 / 13$ & 56616.62 & -60.07 & - & - & - & $>18.59$ & $>18.68$ & - & SMT \\
\hline $25 / 11 / 13$ & 56621.60 & -55.66 & - & - & - & $>20.03$ & $>19.95$ & - & SMT \\
\hline $30 / 11 / 13$ & 56626.58 & -51.26 & - & - & - & $>19.94$ & $>19.91$ & - & SMT \\
\hline $01 / 12 / 13$ & 56627.55 & -50.40 & - & - & - & $>20.30$ & $>19.99$ & - & SMT \\
\hline $06 / 12 / 13$ & 56632.55 & -45.97 & - & - & - & - & $>20.28$ & - & SMT \\
\hline $27 / 12 / 13$ & 56653.51 & -27.42 & - & - & - & $17.86(0.04)$ & $18.14(0.06)$ & - & SMT \\
\hline $31 / 12 / 13$ & 56657.55 & -23.85 & - & - & - & $17.50(0.06)$ & $17.83(0.08)$ & - & $\mathrm{SMT}$ \\
\hline $04 / 01 / 14$ & 56661.48 & -20.37 & - & - & - & $17.33(0.02)$ & $17.61(0.06)$ & - & SMT \\
\hline $04 / 01 / 14$ & 56661.49 & -20.36 & - & - & - & $17.32(0.02)$ & $17.62(0.06)$ & - & $\mathrm{SMT}$ \\
\hline $05 / 01 / 14$ & 56662.50 & -19.47 & - & - & - & $17.27(0.04)$ & $17.52(0.05)$ & - & SMT \\
\hline $07 / 01 / 14$ & 56664.49 & -17.71 & - & - & - & $17.09(0.26)$ & $17.47(0.08)$ & - & $\mathrm{SMT}$ \\
\hline $07 / 01 / 14$ & 56664.50 & -17.70 & - & - & - & $17.20(0.05)$ & $17.43(0.08)$ & - & $\mathrm{SMT}$ \\
\hline $25 / 01 / 14$ & 56682.47 & -1.80 & - & - & - & $16.88(0.04)$ & $17.07(0.08)$ & - & SMT \\
\hline $26 / 01 / 14$ & 56683.51 & -0.88 & - & - & - & $16.89(0.03)$ & $17.09(0.08)$ & - & SMT \\
\hline $26 / 01 / 14$ & 56683.52 & -0.87 & - & - & - & $16.88(0.03)$ & $17.06(0.09)$ & - & SMT \\
\hline $27 / 01 / 14$ & 56684.50 & 0.00 & - & - & - & $16.86(0.27)$ & $17.12(0.13)$ & - & SMT \\
\hline $28 / 01 / 14$ & 56685.49 & 0.88 & - & - & - & $16.89(0.05)$ & $17.12(0.11)$ & - & $\mathrm{SMT}$ \\
\hline $29 / 01 / 14$ & 56686.48 & 1.75 & - & - & - & $16.94(0.04)$ & $17.06(0.10)$ & - & $\mathrm{SMT}$ \\
\hline $29 / 01 / 14$ & 56686.49 & 1.76 & - & - & - & $16.95(0.05)$ & $17.13(0.11)$ & - & $\mathrm{SMT}$ \\
\hline $30 / 01 / 14$ & 56687.48 & 2.64 & - & - & - & $16.95(0.06)$ & $17.09(0.09)$ & - & $\mathrm{SMT}$ \\
\hline $31 / 01 / 14$ & 56688.47 & 3.51 & - & - & - & - & $17.14(0.11)$ & - & SMT \\
\hline $06 / 02 / 14$ & 56694.49 & 8.84 & - & - & - & $17.06(0.05)$ & $17.16(0.09)$ & - & $\mathrm{SMT}$ \\
\hline $19 / 02 / 14$ & 56708.03 & 20.82 & - & - & $16.76(0.02)$ & - & - & - & NTT \\
\hline $20 / 02 / 14$ & 56709.02 & 21.70 & - & - & $16.83(0.01)$ & - & - & - & NTT \\
\hline $21 / 02 / 14$ & 56709.88 & 22.56 & $17.08(0.09)$ & $17.67(0.09)$ & $16.89(0.12)$ & - & - & - & SWIFT \\
\hline $21 / 02 / 14$ & 56710.02 & 22.58 & - & - & $16.86(0.02)$ & - & - & - & NTT \\
\hline $21 / 02 / 14$ & 56711.02 & 23.47 & - & - & $16.87(0.02)$ & - & - & - & NTT \\
\hline $24 / 02 / 14$ & 56712.82 & 25.17 & $17.35(0.08)$ & $17.74(0.10)$ & $17.04(0.19)$ & - & - & - & SWIFT \\
\hline $27 / 02 / 14$ & 56715.36 & 27.43 & $17.48(0.08)$ & $17.91(0.10)$ & $17.21(0.31)$ & - & - & - & SWIFT \\
\hline $28 / 02 / 14$ & 56717.04 & 28.80 & - & - & $17.06(0.02)$ & - & - & - & NTT \\
\hline $03 / 03 / 14$ & 56719.16 & 30.81 & $17.73(0.08)$ & $17.78(0.10)$ & $17.15(0.14)$ & - & - & - & SWIFT \\
\hline $05 / 03 / 14$ & 56721.17 & 32.59 & $17.74(0.09)$ & $17.92(0.10)$ & $17.22(0.14)$ & - & - & - & SWIFT \\
\hline $08 / 03 / 14$ & 56724.56 & 35.61 & $17.96(0.09)$ & $18.07(0.10)$ & $17.29(0.12)$ & - & - & - & SWIFT \\
\hline $08 / 03 / 14$ & 56725.02 & 35.86 & - & - & $17.28(0.02)$ & - & - & - & NTT \\
\hline $09 / 03 / 14$ & 56726.02 & 36.74 & - & - & $17.40(0.02)$ & - & - & - & NTT \\
\hline $10 / 03 / 14$ & 56727.02 & 37.62 & - & - & $17.52(0.02)$ & - & - & - & NTT \\
\hline $06 / 07 / 14$ & 56844.40 & 141.50 & - & $22.30(0.15)$ & $21.80(0.10)$ & $22.32(0.10)$ & $21.80(0.10)$ & $20.70(0.10)$ & $\mathrm{LCO}$ \\
\hline $10 / 07 / 14$ & 56848.40 & 145.04 & - & $22.48(0.15)$ & $22.05(0.10)$ & $22.49(0.10)$ & $21.88(0.10)$ & $20.83(0.10)$ & $\mathrm{LCO}$ \\
\hline $13 / 07 / 14$ & 56851.72 & 147.98 & - & - & - & - & $>21.50$ & - & $\mathrm{LCO}$ \\
\hline $31 / 07 / 14$ & 56869.68 & 163.88 & - & - & - & $>21.29$ & $>20.94$ & - & $\mathrm{SMT}$ \\
\hline $15 / 08 / 14$ & 56885.23 & 177.64 & - & - & - & - & $21.74(0.09)$ & - & NTT \\
\hline $25 / 08 / 14$ & 56895.17 & 186.43 & - & - & - & - & $21.87^{\dagger}(0.06)$ & $21.50(0.05)$ & NTT \\
\hline $21 / 09 / 14$ & 56922.10 & 210.27 & - & - & $23.26(0.19)$ & - & $22.34^{\dagger}(0.06)$ & - & NTT \\
\hline $23 / 09 / 14$ & 56924.15 & 212.08 & - & - & - & $23.72(0.10)$ & $22.54(0.14)$ & $21.80(0.09)$ & $\mathrm{NTT}$ \\
\hline $21 / 10 / 14$ & 56952.15 & 236.86 & - & - & - & $24.75(0.17)$ & $23.23(0.24)$ & $22.35(0.14)$ & NTT \\
\hline $22 / 12 / 14$ & 56975.11 & 257.18 & - & - & - & $24.96(0.26)$ & $23.68(0.29)$ & $22.80(0.19)$ & NTT \\
\hline $\begin{array}{c}18 / 02 / 15 \\
\text { Host A }\end{array}$ & 57072.04 & 344.48 & - & - & - & - & - & $>24.00$ & NTT \\
\hline $20 / 01 / 15$ & 57043.09 & 318.75 & - & - & - & - & $23.26(0.16)$ & - & NTT \\
\hline $\begin{array}{c}10 / 12 / 15 \\
\text { Host B }\end{array}$ & 57367.12 & 606.77 & - & - & - & $24.43(0.16)$ & $23.20(0.16)$ & $21.82(0.16)$ & $\mathrm{NTT}$ \\
\hline $01 / 02 / 16$ & 57420.08 & 653.85 & - & - & - & $24.71(0.38)$ & $24.55(0.35)$ & $23.54(0.32)$ & Magellan \\
\hline $\begin{array}{c}\text { Date } \\
\mathrm{dd} / \mathrm{mm} / \mathrm{yy}\end{array}$ & MJD & $\begin{array}{c}\text { Phase* } \\
\text { (days) }\end{array}$ & & $J$ & $H$ & K & & & Telescope \\
\hline $23 / 11 / 14$ & 56985.12 & 266.03 & & $22.21(0.18)$ & $>22.50$ & $21.48(0.12)$ & & & NTT \\
\hline $20 / 12 / 14$ & 57012.10 & 289.91 & & - & - & $22.10(0.42)$ & & & NTT \\
\hline $\begin{array}{c}29 / 12 / 14 \\
\text { Host A }\end{array}$ & 57021.02 & 297.90 & & - & - & $>22.18$ & & & NTT \\
\hline $17 / 12 / 15$ & 57374.12 & 613.00 & & $>23.10$ & $>23.00$ & $>23.20$ & & & NTT \\
\hline
\end{tabular}

* Phase with respect to the $r$-band maximum.

$\dagger r$ in the AB magnitude system from R-band-filter converted through SNAP, since only these tow observations were done with the $R$ filter. 
Table C2. $g, r, i, z$ magnitudes of PS15br and assigned errors in brackets.

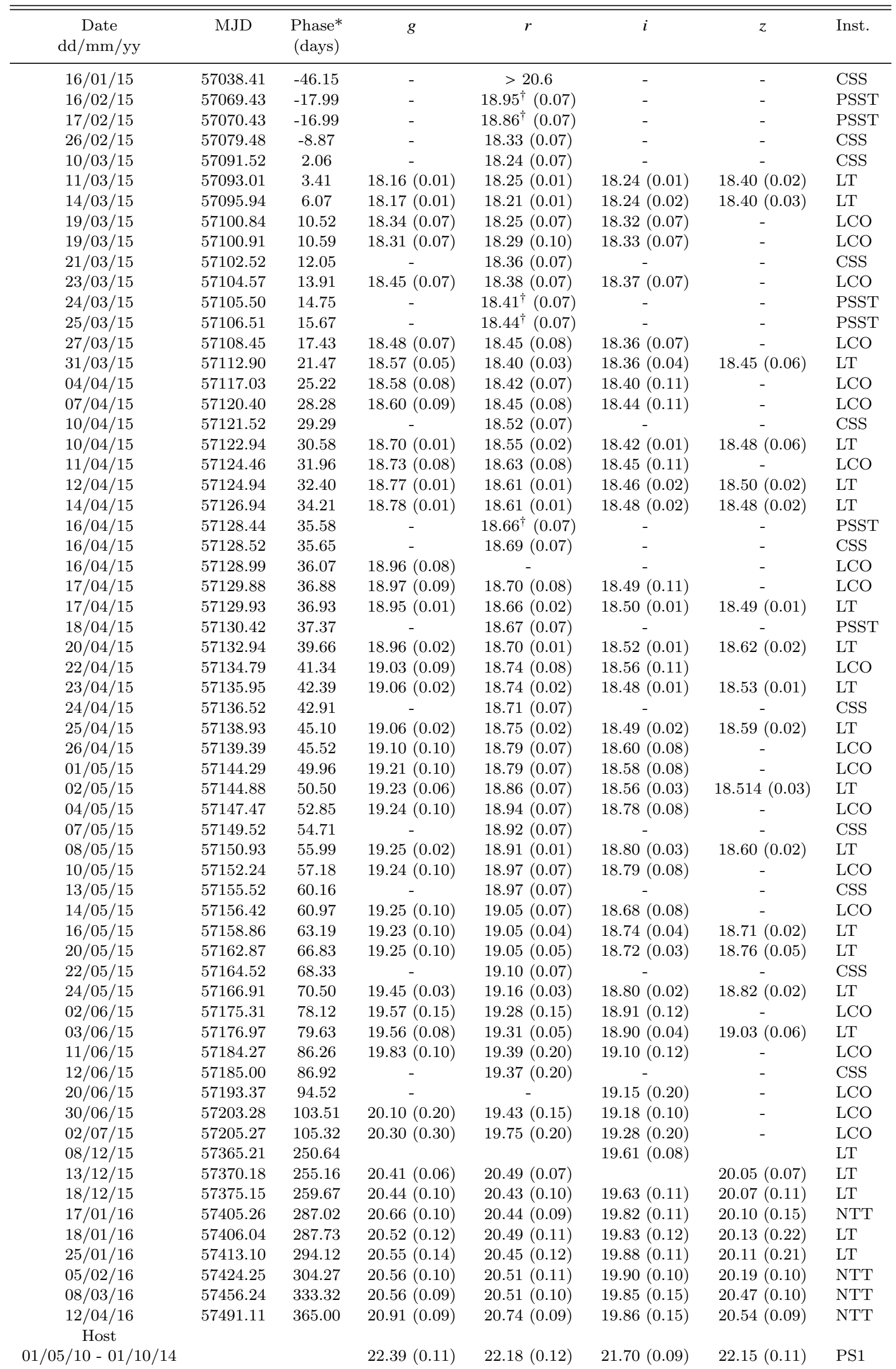


Table C3. $U, B, V, J, H, K$ magnitudes of PS15br and assigned errors in brackets.

\begin{tabular}{|c|c|c|c|c|c|c|}
\hline $\begin{array}{c}\text { Date } \\
\mathrm{dd} / \mathrm{mm} / \mathrm{yy}\end{array}$ & MJD & $\begin{array}{c}\text { Phase* } \\
\text { (days) }\end{array}$ & $U$ & $B$ & V & Inst. \\
\hline $10 / 03 / 15$ & 57092.07 & 2.56 & - & - & $18.00(0.01)$ & NTT \\
\hline $11 / 03 / 15$ & 57092.87 & 3.29 & $17.88(0.10)$ & $18.44(0.10)$ & $18.04(0.13)$ & SWIFT \\
\hline $12 / 03 / 15$ & 57094.23 & 4.52 & - & - & $17.95(0.01)$ & NTT \\
\hline $13 / 03 / 15$ & 57095.20 & 5.40 & - & - & $17.97(0.02)$ & NTT \\
\hline $14 / 03 / 15$ & 57095.96 & 6.09 & $17.95(0.13)$ & $18.48(0.13)$ & $18.05(0.19)$ & SWIFT \\
\hline $19 / 03 / 15$ & 57100.84 & 10.52 & - & $18.51(0.10)$ & $18.10(0.07)$ & $\mathrm{LCO}$ \\
\hline $19 / 03 / 15$ & 57100.91 & 10.58 & - & $18.63(0.08)$ & $18.15(0.07)$ & $\mathrm{LCO}$ \\
\hline $20 / 03 / 15$ & 57102.22 & 11.77 & $18.32(0.11)$ & $18.64(0.12)$ & $18.18(0.21)$ & SWIFT \\
\hline $23 / 03 / 15$ & 57104.57 & 13.91 & - & $18.67(0.08)$ & $18.21(0.07)$ & $\mathrm{LCO}$ \\
\hline $23 / 03 / 15$ & 57105.14 & 14.42 & $18.65(0.15)$ & $18.73(0.19)$ & $18.21(0.21)$ & SWIFT \\
\hline $26 / 03 / 15$ & 57107.93 & 16.96 & $18.80(0.15)$ & $18.83(0.19)$ & $18.30(0.23)$ & SWIFT \\
\hline $27 / 03 / 15$ & 57108.45 & 17.43 & - & $18.82(0.08)$ & $18.34(0.07)$ & $\mathrm{LCO}$ \\
\hline $27 / 03 / 15$ & 57109.30 & 18.20 & - & - & $18.35(0.06)$ & NTT \\
\hline $28 / 03 / 15$ & 57110.04 & 18.87 & - & - & $18.30(0.01)$ & NTT \\
\hline $30 / 03 / 15$ & 57112.15 & 20.79 & $19.02(0.18)$ & $19.01(0.13)$ & $18.38(0.21)$ & SWIFT \\
\hline $04 / 04 / 15$ & 57116.99 & 25.18 & $19.22(0.18)$ & $19.04(0.18)$ & $18.43(0.21)$ & SWIFT \\
\hline $04 / 04 / 15$ & 57117.03 & 25.22 & - & $19.05(0.15)$ & $18.44(0.10)$ & $\mathrm{LCO}$ \\
\hline $07 / 04 / 15$ & 57119.98 & 27.90 & $19.42(0.18)$ & $19.07(0.18)$ & $18.50(0.21)$ & SWIFT \\
\hline $07 / 04 / 15$ & 57120.40 & 28.28 & - & $19.08(0.15)$ & $18.49(0.14)$ & $\mathrm{LCO}$ \\
\hline $11 / 04 / 15$ & 57124.46 & 31.96 & - & $19.17(0.11)$ & $18.58(0.09)$ & $\mathrm{LCO}$ \\
\hline $16 / 04 / 15$ & 57128.99 & 36.07 & - & $19.19(0.13)$ & $18.76(0.10)$ & $\mathrm{LCO}$ \\
\hline $17 / 04 / 15$ & 57129.88 & 36.88 & - & $19.30(0.11)$ & $18.79(0.11)$ & $\mathrm{LCO}$ \\
\hline $18 / 04 / 15$ & 57130.81 & 37.73 & - & $19.45(0.11)$ & $18.80(0.09)$ & $\mathrm{LCO}$ \\
\hline $22 / 04 / 15$ & 57134.79 & 41.34 & - & $19.47(0.20)$ & $18.85(0.11)$ & $\mathrm{LCO}$ \\
\hline $25 / 04 / 15$ & 57138.13 & 44.37 & - & - & $18.84(0.03)$ & NTT \\
\hline $26 / 04 / 15$ & 57139.39 & 45.52 & - & $19.44(0.12)$ & $18.81(0.10)$ & $\mathrm{LCO}$ \\
\hline $01 / 05 / 15$ & 57144.29 & 49.96 & - & $19.43(0.12)$ & $18.84(0.10)$ & $\mathrm{LCO}$ \\
\hline $04 / 05 / 15$ & 57147.47 & 52.85 & - & $19.42(0.12)$ & $18.84(0.10)$ & $\mathrm{LCO}$ \\
\hline $10 / 05 / 15$ & 57152.24 & 57.18 & - & $19.59(0.12)$ & $18.86(0.10)$ & $\mathrm{LCO}$ \\
\hline $14 / 05 / 15$ & 57156.42 & 60.97 & - & $19.67(0.20)$ & $19.15(0.10)$ & $\mathrm{LCO}$ \\
\hline $\begin{array}{c}\text { Date } \\
\mathrm{dd} / \mathrm{mm} / \mathrm{yy}\end{array}$ & $\begin{array}{l}\text { MJD } \\
\text { (days) }\end{array}$ & Phase* & $J$ & $H$ & $K$ & Telescope \\
\hline $11 / 03 / 15$ & 57093.22 & 3.60 & $17.80(0.02)$ & $17.48(0.03)$ & $17.27(0.06)$ & NTT \\
\hline $16 / 04 / 15$ & 57129.14 & 36.21 & $17.76(0.01)$ & $17.23(0.01)$ & $17.01(0.02)$ & NTT \\
\hline $16 / 01 / 16$ & 57404.25 & 286.10 & $19.06(0.05)$ & $18.95(0.05)$ & $17.61(0.08)$ & NTT \\
\hline $06 / 02 / 16$ & 57425.16 & 305.09 & $19.293(0.12)$ & $19.105(0.10)$ & $17.630(0.08)$ & NTT \\
\hline $07 / 03 / 16$ & 57455.22 & 332.40 & $19.098(0.25)$ & $19.239(0.08)$ & $17.537(0.12)$ & NTT \\
\hline $13 / 04 / 16$ & 57492.09 & 365.89 & $19.425(0.12)$ & $19.514(0.18)$ & $17.659(0.08)$ & NTT \\
\hline
\end{tabular}


Table C4. Swift+UVOT uvw2, uvm2, uvw1 magnitudes of SN2013hx and PS15br and assigned errors in brackets.

\begin{tabular}{|c|c|c|c|c|c|}
\hline $\begin{array}{c}\text { Date } \\
\mathrm{dd} / \mathrm{mm} / \mathrm{yy}\end{array}$ & MJD & $\begin{array}{c}\text { Phase* } \\
\text { (days) }\end{array}$ & $u v w 2$ & uvm 2 & $u v w 1$ \\
\hline \multicolumn{6}{|c|}{ SN2013hx } \\
\hline $21 / 02 / 14$ & 56709.88 & 22.56 & 17.67 .10 & 17.44 .08 & 17.17 .09 \\
\hline $24 / 02 / 14$ & 56712.82 & 25.17 & 17.87 .10 & 17.75 .08 & 17.46 .09 \\
\hline $27 / 02 / 14$ & 56715.36 & 27.43 & 18.10 .12 & 18.05 .16 & 17.63 .09 \\
\hline $03 / 03 / 14$ & 56719.16 & 30.81 & 18.49 .12 & 18.13 .10 & 18.01 .11 \\
\hline $05 / 03 / 14$ & 56721.17 & 32.59 & 18.58 .13 & 18.38 .11 & 18.07 .13 \\
\hline 08/03/14 & 56724.56 & 35.61 & 18.85 .13 & 18.58 .11 & 18.34 .12 \\
\hline $27 / 04 / 14$ & 56774.90 & 80.35 & - & $>21.0$ & - \\
\hline $28 / 04 / 14$ & 56775.03 & 80.47 & - & - & $>21.8$ \\
\hline $30 / 04 / 14$ & 56777.36 & 82.54 & $>22.0$ & - & - \\
\hline $07 / 07 / 14$ & 56845.79 & 143.37 & $>21.9$ & - & - \\
\hline $21 / 07 / 14$ & 56859.07 & 155.17 & - & - & $>21.6$ \\
\hline $24 / 07 / 14$ & 56862.46 & 158.19 & - & $>21.1$ & - \\
\hline \multicolumn{6}{|c|}{ PS15br } \\
\hline $11 / 03 / 15$ & 57092.87 & 3.29 & $18.70(0.11)$ & $18.67(0.17)$ & $18.25(0.11)$ \\
\hline $14 / 03 / 15$ & 57095.96 & 6.09 & $19.01(0.17)$ & $18.87(0.22)$ & $18.50(0.18)$ \\
\hline $20 / 03 / 15$ & 57102.22 & 11.77 & $19.37(0.14)$ & $19.24(0.19)$ & $18.83(0.15)$ \\
\hline $23 / 03 / 15$ & 57105.14 & 14.42 & $19.57(0.21)$ & $19.44(0.32)$ & $18.85(0.15)$ \\
\hline $26 / 03 / 15$ & 57107.93 & 16.96 & $19.78(0.18)$ & $19.13(0.19)$ & $18.92(0.16)$ \\
\hline $30 / 03 / 15$ & 57112.15 & 20.79 & $20.06(0.19)$ & $19.77(0.24)$ & $19.29(0.18)$ \\
\hline $04 / 04 / 15$ & 57116.99 & 25.18 & $20.26(0.30)$ & $19.94(0.26)$ & $19.47(0.24)$ \\
\hline $07 / 04 / 15$ & 57119.98 & 27.90 & $20.39(0.24)$ & $20.03(0.28)$ & $19.62(0.28)$ \\
\hline $11 / 11 / 15$ & 57337.64 & 225.60 & $21.15(0.23)$ & - & \\
\hline $15 / 11 / 15$ & 57341.03 & 228.68 & $20.92(0.24)$ & - & \\
\hline $21 / 01 / 16$ & 57407.80 & 289.33 & - & - & $20.98(0.38)$ \\
\hline $25 / 01 / 16$ & 57412.04 & 293.18 & $21.03(0.27)$ & $21.04(0.30)$ & $20.65(0.25)$ \\
\hline
\end{tabular}

* Phase with respect to the r-band maximum.

Table C5. Magnitudes in $B, V, g, r, i$ of the local sequence stars in the field of SN2013hx.

\begin{tabular}{lccccc}
\hline \hline Star & $B$ & $V$ & $g$ & $r$ & $i$ \\
\hline 1 & $21.06(0.09)$ & $19.24(0.05)$ & $19.35(0.03)$ & $17.81(0.03)$ & $16.55(0.02)$ \\
2 & $18.96(0.06)$ & $16.68(0.02)$ & $16.83(0.02)$ & $15.26(0.03)$ & $13.81(0.02)$ \\
3 & $19.59(0.07)$ & $18.17(0.03)$ & $17.98(0.02)$ & $17.30(0.02)$ & $16.69(0.02)$ \\
4 & - & $22.89(0.09)$ & $22.90(0.08)$ & $21.17(0.08)$ & $20.65(0.05)$ \\
5 & $20.56(0.08)$ & $18.76(0.05)$ & $18.90(0.04)$ & $17.39(0.05)$ & $15.94(0.02)$ \\
6 & $19.29(0.07)$ & $17.95(0.06)$ & $17.72(0.03)$ & $17.20(0.03)$ & $16.65(0.03)$ \\
7 & - & $22.50(0.09)$ & $22.17(0.06)$ & $20.41(0.05)$ & $18.40(0.04)$ \\
8 & - & $22.50(0.08)$ & $22.61(0.09)$ & $21.82(0.08)$ & $21.34(0.06)$ \\
9 & - & $22.54(0.08)$ & $22.77(0.09)$ & $21.11(0.07)$ & $19.13(0.04)$ \\
10 & $17.42(0.05)$ & $16.04(0.02)$ & $15.83(0.02)$ & $15.15(0.02)$ & $14.67(0.03)$ \\
11 & - & $22.22(0.08)$ & $22.38(0.08)$ & $21.07(0.07)$ & $20.21(0.05)$ \\
12 & - & $22.36(0.08)$ & $22.50(0.09)$ & $21.15(0.07)$ & $20.28(0.05)$ \\
\hline
\end{tabular}


Table C6. Magnitudes in $B, V, g, r, i, z$ of the local sequence stars in the field of PS15br.

\begin{tabular}{lcccccc}
\hline \hline Star & $B$ & $V$ & $g$ & $r$ & $i$ & $z$ \\
\hline 1 & $16.50(0.02)$ & $16.02(0.02)$ & $16.19(0.01)$ & $15.96(0.01)$ & $15.83(0.02)$ & $15.83(0.01)$ \\
2 & $18.02(0.02)$ & $17.47(0.02)$ & $17.67(0.01)$ & $17.38(0.01)$ & $17.22(0.01)$ & $17.18(0.01)$ \\
3 & $20.57(0.05)$ & $19.32(0.03)$ & $19.86(0.02)$ & $18.85(0.01)$ & $18.27(0.01)$ & $17.97(0.01)$ \\
4 & $17.41(0.02)$ & $16.51(0.02)$ & $16.91(0.01)$ & $16.16(0.01)$ & $15.85(0.01)$ & $15.69(0.01)$ \\
5 & $19.03(0.03)$ & $17.89(0.02)$ & $18.41(0.01)$ & $17.44(0.01)$ & $16.97(0.01)$ & $16.72(0.01)$ \\
6 & $19.12(0.03)$ & $18.65(0.03)$ & $18.81(0.02)$ & $18.60(0.01)$ & $18.49(0.01)$ & $18.47(0.01)$ \\
7 & $18.33(0.03)$ & $17.80(0.02)$ & $17.99(0.01)$ & $17.70(0.01)$ & $17.55(0.01)$ & $17.52(0.01)$ \\
8 & $20.17(0.05)$ & $18.51(0.03)$ & $19.07(0.02)$ & $17.89(0.01)$ & $16.96(0.01)$ & $16.49(0.01)$ \\
9 & $18.07(0.02)$ & $17.44(0.02)$ & $17.68(0.01)$ & $17.29(0.01)$ & $17.08(0.01)$ & $17.01(0.01)$ \\
10 & $20.75(0.05)$ & $19.59(0.05)$ & $20.09(0.02)$ & $19.15(0.01)$ & $18.63(0.01)$ & $18.37(0.01)$ \\
11 & $21.48(0.06)$ & $20.15(0.05)$ & $20.72(0.03)$ & $19.63(0.02)$ & $19.03(0.02)$ & $18.66(0.01)$ \\
\hline
\end{tabular}

Table C7. Journal of spectroscopic observations.

\begin{tabular}{|c|c|c|c|c|c|}
\hline $\begin{array}{c}\text { Date } \\
\mathrm{dd} / \mathrm{mm} / \mathrm{yy}\end{array}$ & MJD & $\begin{array}{l}\text { Phase* } \\
\text { (days) }\end{array}$ & $\begin{array}{c}\text { Range }_{(\AA)}^{\dagger} \\
\text { (A) }\end{array}$ & $\begin{array}{l}\text { Resolution } \\
(\AA)\end{array}$ & $\begin{array}{l}\text { Instrumental } \\
\text { Configuration }\end{array}$ \\
\hline \multicolumn{6}{|c|}{ SN2013hx } \\
\hline $31 / 01 / 14$ & 56688.43 & 3.49 & $3100-6900$ & $1.5 / 2.5$ & ANU $2.3 \mathrm{~m}+\mathrm{WiFeS}+\mathrm{B} 300 / \mathrm{R} 300$ \\
\hline $19 / 02 / 14$ & 56708.03 & 20.82 & $3600-9300$ & 18 & $\mathrm{NTT}+\mathrm{EFOSC} 2+\mathrm{gm} 13$ \\
\hline $20 / 02 / 14$ & 56709.02 & 21.70 & $3600-9300$ & 18 & $\mathrm{NTT}+\mathrm{EFOSC} 2+\mathrm{gm} 13$ \\
\hline $21 / 02 / 14$ & 56710.02 & 22.58 & $6000-10000$ & 16 & $\mathrm{NTT}+\mathrm{EFOSC} 2+\mathrm{gm} 16$ \\
\hline $22 / 02 / 14$ & 56711.02 & 23.47 & $3300-7500$ & 13 & $\mathrm{NTT}+\mathrm{EFOSC} 2+\mathrm{gm} 11$ \\
\hline $24 / 02 / 14$ & 56712.44 & 24.84 & $3400-6900$ & $1.5 / 2.5$ & ANU $2.3 \mathrm{~m}+\mathrm{WiFeS}+\mathrm{B} 300 / \mathrm{R} 300$ \\
\hline $28 / 02 / 14$ & 56717.04 & 28.80 & $3300-10000$ & $13 / 16$ & NTT+EFOSC2+gm11/gm16 \\
\hline $04 / 03 / 14$ & 56720.42 & 31.93 & $3400-6900$ & $1.5 / 2.5$ & ANU $2.3 \mathrm{~m}+\mathrm{WiFeS}+\mathrm{B} 300 / \mathrm{R} 300$ \\
\hline $08 / 03 / 14$ & 56725.03 & 35.87 & $6000-10000$ & 16 & $\mathrm{NTT}+\mathrm{EFOSC} 2+\mathrm{gm} 16$ \\
\hline $09 / 03 / 14$ & 56726.06 & 36.78 & $3300-10000$ & $13 / 16$ & $\mathrm{NTT}+\mathrm{EFOSC} 2+\mathrm{gm} 11 / \mathrm{gm} 16$ \\
\hline $10 / 03 / 14$ & 56727.02 & 37.62 & $3300-7500$ & 13 & $\mathrm{NTT}+\mathrm{EFOSC} 2+\mathrm{gm} 11$ \\
\hline $14 / 03 / 14$ & 56730.39 & 40.79 & $3400-6900$ & $1.5 / 2.5$ & ANU $2.3 \mathrm{~m}+\mathrm{WiFeS}+\mathrm{B} 300 / \mathrm{R} 300$ \\
\hline $21 / 09 / 14$ & 56922.10 & 210.27 & $6900-7700$ & 18 & $\mathrm{NTT}+\mathrm{EFOSC} 2+\mathrm{gm} 13$ \\
\hline $22 / 10 / 14$ & 56953.07 & 237.67 & $4500-9300$ & 11 & VLT+FORS2+GRIS_300V \\
\hline \multicolumn{6}{|c|}{ PS15br } \\
\hline $10 / 03 / 15$ & 57092.07 & 2.56 & $3600-9300$ & 18 & $\mathrm{NTT}+\mathrm{EFOSC} 2+\mathrm{gm} 13$ \\
\hline $12 / 03 / 15$ & 57093.56 & 3.91 & $4100-6900$ & $1.5 / 2.5$ & ANU $2.3 \mathrm{~m}+\mathrm{WiFeS}+\mathrm{B} 300 / \mathrm{R} 300$ \\
\hline $12 / 03 / 15$ & 57094.23 & 4.52 & $3300-7500$ & 13 & $\mathrm{NTT}+\mathrm{EFOSC} 2+\mathrm{gm} 11$ \\
\hline $13 / 03 / 15$ & 57095.20 & 5.40 & $6000-10000$ & 16 & $\mathrm{NTT}+\mathrm{EFOSC} 2+\mathrm{gm} 16$ \\
\hline $27 / 03 / 15$ & 57109.30 & 18.20 & $3300-10000$ & 18 & $\mathrm{NTT}+\mathrm{EFOSC} 2+\mathrm{gm} 13$ \\
\hline $28 / 03 / 15$ & 57100.05 & 18.88 & $3600-9300$ & 18 & $\mathrm{NTT}+\mathrm{EFOSC} 2+\mathrm{gm} 13$ \\
\hline $12 / 04 / 15$ & 57125.06 & 32.51 & $3600-9300$ & 18 & $\mathrm{NTT}+\mathrm{EFOSC} 2+\mathrm{gm} 13$ \\
\hline $25 / 04 / 15$ & 57138.13 & 44.37 & $3600-9300$ & 18 & $\mathrm{NTT}+\mathrm{EFOSC} 2+\mathrm{gm} 13$ \\
\hline $29 / 06 / 15$ & 57202.13 & 102.47 & $3600-9300$ & $4.3 / 5.8$ & $\mathrm{UH} 2.2 \mathrm{~m}+\mathrm{SNIFS}+\mathrm{B} / \mathrm{R}$ \\
\hline $08 / 01 / 16$ & 57396.24 & 278.82 & $3600-9300$ & 18 & $\mathrm{NTT}+\mathrm{EFOSC} 2+\mathrm{gm} 13$ \\
\hline $12 / 03 / 16$ & 57460.26 & 336.98 & $2811-24511$ & $1.0 / 1.1 / 3.3$ & VLT+XSHOOTER+UV/OPT/NIR \\
\hline $09 / 04 / 16$ & 57488.08 & 362.24 & $2811-24511$ & $1.0 / 1.1 / 3.3$ & VLT+XSHOOTER+UV/OPT/NIR \\
\hline $10 / 04 / 16$ & 57489.09 & 363.16 & $3600-9300$ & 18 & $\mathrm{NTT}+\mathrm{EFOSC} 2+\mathrm{gm} 13$ \\
\hline
\end{tabular}

* Phase with respect to the r-band maximum.

$\dagger$ Range with respect to the observed frame. 

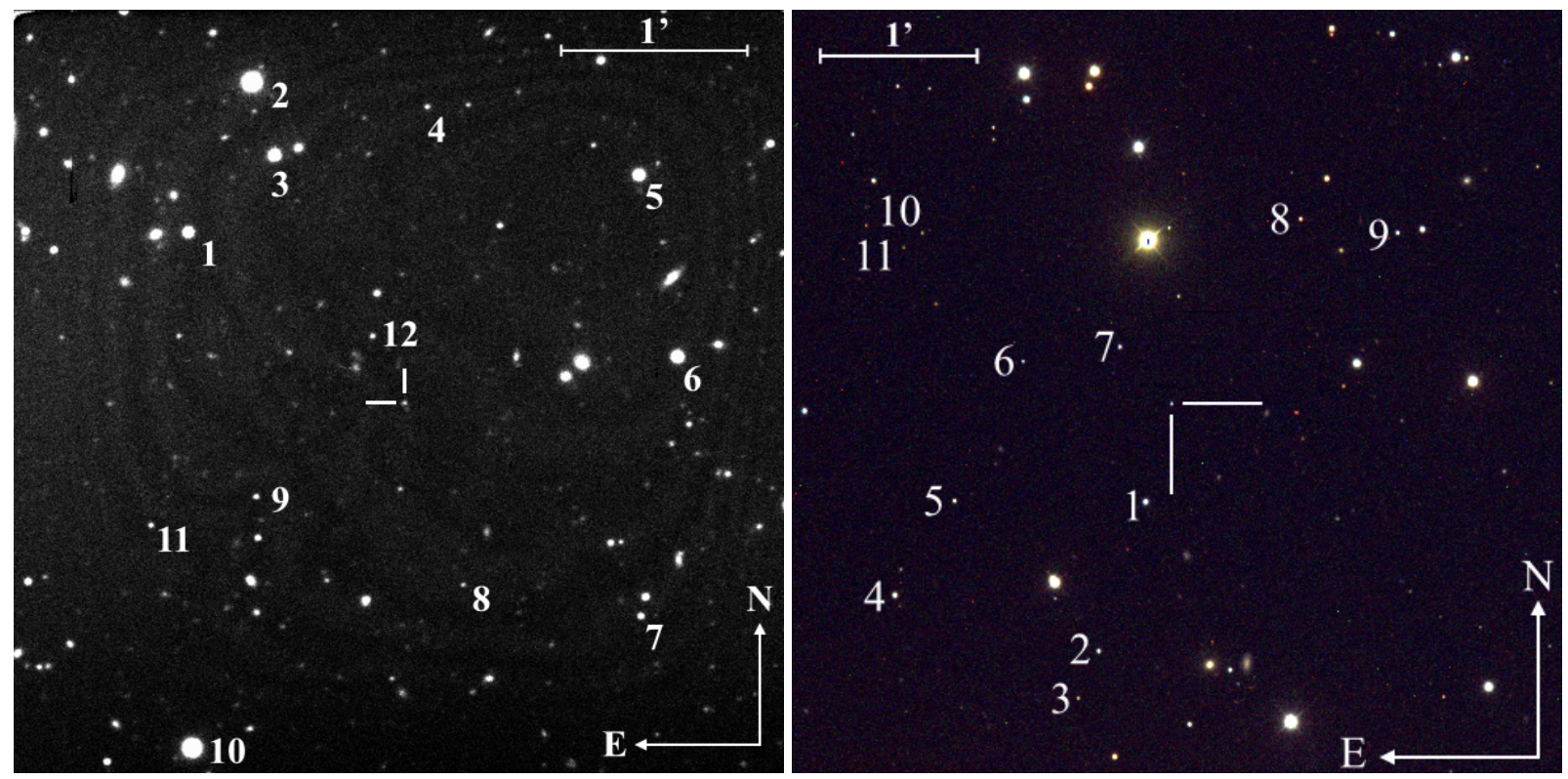

Figure D1. Left: NTT + EFOSC2 $+r$ image of SN2013hx. The SN position is indicated with cross white marks. The sequence of stars in the field used to calibrate the optical and NIR magnitudes of SN2013hx is indicated. Right: LT $+\mathrm{IO}+g / r / i$ image of PS15br (cross white marks). The sequence of stars in the field used to calibrate the optical and NIR magnitudes of PS15br is indicated 